\title{
DECOHERENCE, EINSELECTION, AND THE QUANTUM ORIGINS OF THE CLASSICAL
}

\author{
Wojciech Hubert Zurek \\ Theory Division, LANL, Mail Stop B288 \\ Los Alamos, New Mexico 87545
}

Decoherence is caused by the interaction with the environment which in effect monitors certain observables of the system, destroying coherence between the pointer states corresponding to their eigenvalues. This leads to environment-induced superselection or einselection, a quantum process associated with selective loss of information. Einselected pointer states are stable. They can retain correlations with the rest of the Universe in spite of the environment. Einselection enforces classicality by imposing an effective ban on the vast majority of the Hilbert space, eliminating especially the flagrantly nonlocal "Schrödinger cat" states. Classical structure of phase space emerges from the quantum Hilbert space in the appropriate macroscopic limit: Combination of einselection with dynamics leads to the idealizations of a point and of a classical trajectory. In measurements, einselection replaces quantum entanglement between the apparatus and the measured system with the classical correlation. Only the preferred pointer observable of the apparatus can store information that has predictive power. When the measured quantum system is microscopic and isolated, this restriction on the predictive utility of its correlations with the macroscopic apparatus results in the effective "collapse of the wavepacket". Existential interpretation implied by einselection regards observers as open quantum systems, distinguished only by their ability to acquire, store, and process information. Spreading of the correlations with the effectively classical pointer states throughout the environment allows one to understand 'classical reality' as a property based on the relatively objective existence of the einselected states: They can be "found out" without being re-prepared, e.g, by intercepting the information already present in the environment. The redundancy of the records of pointer states in the environment (which can be thought of as their 'fitness' in the Darwinian sense) is a measure of their classicality. A new symmetry appears in this setting: Environment - assisted invariance or envariance sheds a new light on the nature of ignorance of the state of the system due to quantum correlations with the environment, and leads to Born's rules and to the reduced density matrices, ultimately justifying basic principles of the program of decoherence and einselection.

\section{Contents}

I. INTRODUCTION

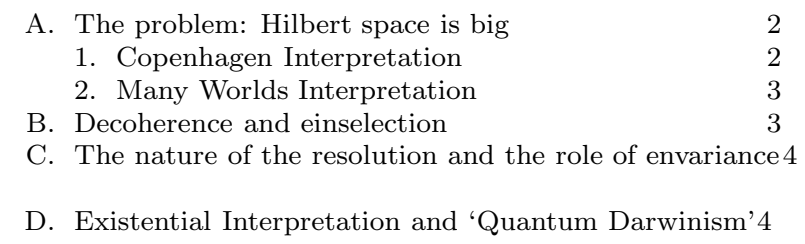
II. QUANTUM MEASUREMENTS
A. Quantum conditional dynamics
1. Controlled not and a bit-by-bit measurement
2. Measurements and controlled shifts.
3. Amplification
B. Information transfer in measurements
1. Action per bit
C. "Collapse" analogue in a classical measurement

III. CHAOS AND LOSS OF CORRESPONDENCE 11

A. Loss of the quantum-classical correspondence 11

B. Moval bracket and Liouville flow 12

C. Svmptoms of correspondence loss 13

1. Expectation values 13

2. Structure saturation 13

IV. ENVIRONMENT - INDUCED SUPERSELECTION 14

A. Models of einselection 14

1. Decoherence of a single qubit 15

2. The classical domain and a guantum hald $\quad 16$

3. Einselection and controlled shifts 16

B. Einselection as the selective loss of information $\quad 17$

1. Mutual information and discord 18

C. Decoherence, entanglement, dephasing. and noise 19

D. Predictabilitv sieve and einselection 20

V. EINSELECTION IN PHASE SPACE 21

A. Quantum Brownian motion 21

B. Decoherence in quantum Brownian motion 23

1. Decoherence timescale 24

2. Phase space view of decoherence 25

C. Predictability sieve in phase space 26

D. Classical limit in phase space 26

1. Mathematical approach $(\hbar \rightarrow 0) \quad 27$

2. Phvsical approach: The macroscopic limit 27

3. Ignorance inspires confidence in classicality 28

E. Decoherence, chaos, and the Second Law 28

1. Restoration of correspondence 28

2. Entropy production 29

3. Quantum predictability horizon 30

VI. EINSELECTION AND MEASUREMENTS 30

A. Objective existence of einselected states 30

B. Measurements and memories 31

C. Axioms of quantum measurement theory 32

1. Observables are Hermitean - axiom (iiia) 32

2. Eigenvalues as outcomes - axiom (iiib) 33

$\begin{array}{ll}\text { 3. Immediate repeatabilitv, axiom (iv) } & 33 \\ \text { 4. Probabilities. einselection and records } & 34\end{array}$ 


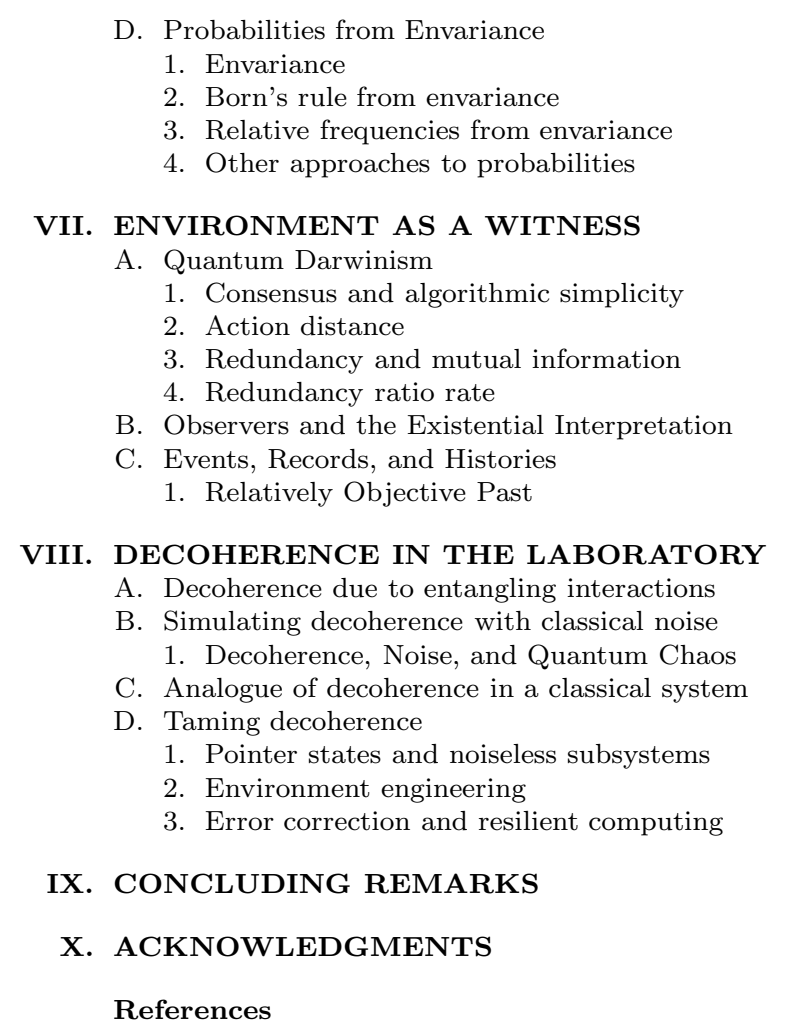

\section{INTRODUCTION}

The issue of interpretation is as old as quantum theory. It dates back to the discussions of Niels Bohr, Werner Heisenberg, Erwin Schrödinger, (Bohr, 1928; 1949; Heisenberg, 1927; Schrödinger, 1926; 1935a,b; see also Jammer, 1974; Wheeler and Zurek, 1983). Perhaps the most incisive critique of the (then new) theory was due to Albert Einstein, who, searching for inconsistencies, distilled the essence of the conceptual difficulties of quantum mechanics through ingenious "gedankenexperiments". We owe him and Bohr clarification of the significance of the quantum indeterminacy in course of the Solvay congress debates (see Bohr, 1949) and elucidation of the nature of quantum entanglement (Einstein, Podolsky, and Rosen, 1935; Bohr, 1935, Schrödinger, 1935a,b). Issues identified then are still a part of the subject.

Within the past two decades the focus of the research on the fundamental aspects of quantum theory has shifted from esoteric and philosophical to more "down to earth" as a result of three developments. To begin with, many of the old gedankenexperiments (such as the EPR "paradox") became compelling demonstrations of quantum physics. More or less simultaneously the role of decoherence begun to be appreciated and einselection was recognized as key in the emergence of classicality. Last not least, various developments have led to a new view of the role of information in physics. This paper reviews progress with a focus on decoherence, einselection and the emergence of classicality, but also attempts a "preview" of the future of this exciting and fundamental area.

\section{A. The problem: Hilbert space is big}

The interpretation problem stems from the vastness of the Hilbert space, which, by the principle of superposition, admits arbitrary linear combinations of any states as a possible quantum state. This law, thoroughly tested in the microscopic domain, bears consequences that defy classical intuition: It appears to imply that the familiar classical states should be an exceedingly rare exception. And, naively, one may guess that superposition principle should always apply literally: Everything is ultimately made out of quantum "stuff". Therefore, there is no a priori reason for macroscopic objects to have definite position or momentum. As Einstein noted ${ }^{1}$ localization with respect to macrocoordinates is not just independent, but incompatible with quantum theory. How can one then establish correspondence between the quantum and the familiar classical reality?

\section{Copenhagen Interpretation}

Bohr's solution was to draw a border between the quantum and the classical and to keep certain objects especially measuring devices and observers - on the classical side (Bohr, 1928; 1949). The principle of superposition was suspended "by decree" in the classical domain. The exact location of this border was difficult to pinpoint, but measurements "brought to a close" quantum events. Indeed, in Bohr's view the classical domain was more fundamental: Its laws were self-contained (they could be confirmed from within) and established the framework necessary to define the quantum.

The first breach in the quantum-classical border appeared early: In the famous Bohr - Einstein double-slit debate, quantum Heisenberg uncertainty was invoked by Bohr at the macroscopic level to preserve wave-particle duality. Indeed, as the ultimate components of classical objects are quantum, Bohr emphasized that the boundary must be moveable, so that even the human nervous system could be regarded as quantum provided that suitable classical devices to detect its quantum features were available. In the words of John Archibald Wheeler (1978; 1983) who has elucidated Bohr's position and decisively contributed to the revival of interest in these matters, "No [quantum] phenomenon is a phenomenon until it is a recorded (observed) phenomenon".

\footnotetext{
${ }^{1}$ In a letter dated 1954, Albert Einstein wrote to Max Born "Let $\psi_{1}$ and $\psi_{2}$ be solutions of the same Schrödinger equation..... When the system is a macrosystem and when $\psi_{1}$ and $\psi_{2}$ are 'narrow' with respect to the macrocoordinates, then in by far the greater number of cases this is no longer true for $\psi=\psi_{1}+\psi_{2}$. Narrowness with respect to macrocoordinates is not only independent of the principles of quantum mechanics, but, moreover, incompatible with them." (The translation from Born (1969) quoted here is due to Joos (1986).)
} 
This is a pithy summary of a point of view - known as the Copenhagen Interpretation (CI) - that has kept many a physicist out of despair. On the other hand, as long as a compelling reason for the quantum-classical border could not be found, the CI Universe would be governed by two sets of laws, with poorly defined domains of jurisdiction. This fact has kept many a student, not to mention their teachers, in despair (Mermin 1990a; b; 1994).

\section{Many Worlds Interpretation}

The approach proposed by Hugh Everett (1957a, b) and elucidated by Wheeler (1957), Bryce DeWitt (1970) and others (see DeWitt and Graham, 1973; Zeh, 1970; 1973; Geroch, 1984; Deutsch, 1985, 1997, 2001) was to enlarge the quantum domain. Everything is now represented by a unitarily evolving state vector, a gigantic superposition splitting to accommodate all the alternatives consistent with the initial conditions. This is the essence of the Many Worlds Interpretation (MWI). It does not suffer from the dual nature of CI. However, it also does not explain the emergence of classical reality.

The difficulty many have in accepting MWI stems from its violation of the intuitively obvious "conservation law" - that there is just one Universe, the one we perceive. But even after this question is dealt with, , many a convert from CI (which claims allegiance of a majority of physicists) to MWI (which has steadily gained popularity; see Tegmark and Wheeler, 2001, for an assessment) eventually realizes that the original MWI does not address the "preferred basis question" posed by Einstein ${ }^{1}$ (see Wheeler, 1983; Stein, 1984; Bell 1981, 1987; Kent, 1990; for critical assessments of MWI). And as long as it is unclear what singles out preferred states, perception of a unique outcome of a measurement and, hence, of a single Universe cannot be explained either ${ }^{2}$.

In essence, Many Worlds Interpretation does not address but only postpones the key question. The quantum - classical boundary is pushed all the way towards the observer, right against the border between the material Universe and the "consciousness", leaving it at a very uncomfortable place to do physics. MWI is incomplete: It does not explain what is effectively classical and why. Nevertheless, it was a crucial conceptual breakthrough:

\footnotetext{
2 DeWitt, in the Many Worlds re-analysis of quantum measurements makes this clear: in DeWitt and Graham (1973), last paragraph of p. 189 he writes about the key 'remaining problem': "Why is it so easy to find apparata in states [with a well defined value of the pointer observable]? In the case of macroscopic apparata it is well known that a small value for the mean square deviation of a macroscopic observable is a fairly stable property of the apparatus. But how does the mean square deviation become so small in the first place? Why is a large value of the mean square deviation of a macroscopic observable virtually never, in fact, encountered in practice? ... a proof of this does not yet exist. It remains a program for the future."
}

Everett reinstated quantum mechanics as a basic tool in the search for its interpretation.

\section{B. Decoherence and einselection}

Decoherence and einselection are two complementary views of the consequences of the same process of environmental monitoring. Decoherence is the destruction of quantum coherence between preferred states associated with the observables monitored by the environment. Einselection is its consequence - the de facto exclusion of all but a small set, a classical domain consisting of pointer states - from within a much larger Hilbert space. Einselected states are distinguished by their resilience stability in spite of the monitoring environment.

The idea that "openness" of quantum systems may have anything to do with the transition from quantum to classical was resolutely ignored for a very long time, probably because in classical physics problems of fundamental importance were always settled in isolated systems. In the context of measurements Gottfried (1967) anticipated some of the latter developments. The fragility of energy levels of quantum systems was emphasized by seminal papers of Dieter Zeh $(1970 ; 1973)$, who argued (inspired by remarks relevant to what would be called today "deterministic chaos" (Borel, 1914)) that macroscopic quantum systems are in effect impossible to isolate.

The understanding of how the environment distills the classical essence from quantum systems is more recent (Zurek, 1981; 1982; 1993a). It combines two observations: (1) In quantum physics "reality" can be attributed to the measured states. (2) Information transfer usually associated with measurements is a common result of almost any interaction with the environment of a system.

Some quantum states are resilient to decoherence. This is the basis of einselection. Using Darwinian analogy, one might say that pointer states are most 'fit'. They survive monitoring by the environment to leave 'descendants' that inherit their properties. Classical domain of pointer states offers a static summary of the result of quantum decoherence. Save for classical dynamics, (almost) nothing happens to these einselected states, even though they are immersed in the environment.

It is difficult to catch einselection "in action": Environment has little effect on the pointer states, as they are already classical. Therefore, it was easy to miss decoherence - driven dynamics of einselection by taking for granted its result - existence of the classical domain, and a ban on arbitrary quantum superpositions. Macroscopic superpositions of einselected states disappear rapidly. Einselection creates effective superselection rules (Wick, Wightman and Wigner, 1952; 1970; Wightman, 1995). However, in the microscopic, decoherence can be slow in comparison with the dynamics.

Einselection is a quantum phenomenon. Its essence cannot be even motivated classically: In classical physics arbitrarily accurate measurements (also by the environ- 
ment) can be in principle carried out without disturbing the system. Only in quantum mechanics acquisition of information inevitably alters the state of the system the fact that becomes apparent in double-slit and related experiments (Wootters and Zurek, 1979; Zurek, 1983).

Quantum nature of decoherence and the absence of classical analogues are a source of misconceptions. For instance, decoherence is sometimes equated with relaxation or classical noise that can be also introduced by the environment. Indeed, all of these effects often appear together and as a consequence of the "openness". The distinction between them can be briefly summed up: Relaxation and noise are caused by the environment perturbing the system, while decoherence and einselection are caused by the system perturbing the environment.

Within the past few years decoherence and einselection became familiar to many. This does not mean that their implications are universally accepted (see comments in the April 1993 issue of Physics Today; d'Espagnat, 1989 and 1995; Bub, 1997; Leggett, 1998 and 2002; Stapp, 2001; exchange of views between Anderson, 2001, and Adler, 2001). In a field where controversy reigned for so long this resistance to a new paradigm is no surprise.

\section{The nature of the resolution and the role of envariance}

Our aim is to explain why does the quantum Universe appear classical. This question can be motivated only in the context of the Universe divided into systems, and must be phrased in the language of the correlations between them. In the absence of systems Schrödinger equation dictates deterministic evolution;

$$
|\Psi(t)\rangle=\exp (-i H t / \hbar)|\Psi(0)\rangle
$$

and the problem of interpretation seems to disappear.

There is no need for "collapse" in a Universe with no systems. Yet, the division into systems is imperfect. As a consequence, the Universe is a collection of open (interacting) quantum systems. As the interpretation problem does not arise in quantum theory unless interacting systems exist, we shall also feel free to assume that an environment exists when looking for a resolution.

Decoherence and einselection fit comfortably in the context of the Many Worlds Interpretation where they define the "branches" of the universal state vector. Decoherence makes MWI complete: It allows one to analyze the Universe as it is seen by an observer, who is also subject to decoherence. Einselection justifies elements of Bohr's CI by drawing the border between the quantum and the classical. This natural boundary can be sometimes shifted: Its effectiveness depends on the degree of isolation and on the manner in which the system is probed, but it is a very effective quantum - classical border nevertheless.

Einselection fits either MWI or CI framework: It supplies a statute of limitations, putting an end to the quantum jurisdiction. . It delineates how much of the
Universe will appear classical to observers who monitor it from within, using their limited capacity to acquire, store, and process information. It allows one to understand classicality as an idealization that holds in the limit of macroscopic open quantum systems.

Environment imposes superselection rules by preserving part of the information that resides in the correlations between the system and the measuring apparatus (Zurek, 1981, 1982). The observer and the environment compete for the information about the system. Environment because of its size and its incessant interaction with the system - wins that competition, acquiring information faster and more completely than the observer. Thus, a record useful for the purpose of prediction must be restricted to the observables that are already monitored by the environment. In that case, the observer and the environment no longer compete and decoherence becomes unnoticeable. Indeed, typically observers use environment as a "communication channel", and monitor it to find out about the system.

Spreading of the information about the system through the environment is ultimately responsible for the emergence of the "objective reality". Objectivity of a state can be quantified by the redundancy with which it is recorded throughout Universe. Intercepting fragments of the environment allows observers to find out (pointer) state of the system without perturbing it (Zurek, 1993a, 1998a, and 2000; see especially section VII of this paper for a preview of this new "environment as a witness" approach to the interpretation of quantum theory).

When an effect of a transformation acting on a system can be undone by a suitable transformation acting on the environment, so that the joint state of the two remains unchanged, the transformed property of the system is said to exhibit "environment assisted invariance" or envariance (Zurek, 2002b). Observer must be obviously ignorant of the envariant properties of the system. Pure entangled states exhibit envariance. Thus, in quantum physics perfect information about the joint state of the system-environment pair can be used to prove ignorance of the state of the system.

Envariance offers a new fundamental view of what is information and what is ignorance in the quantum world. It leads to Born's rule for the probabilities and justifies the use of reduced density matrices as a description of a part of a larger combined system. Decoherence and einselection rely on reduced density matrices. Envariance provides a fundamental resolution of many of the interpretational issues. It will be discussed in section VI D.

\section{Existential Interpretation and 'Quantum Darwinism'}

What the observer knows is inseparable from what the observer is: The physical state of his memory implies his information about the Universe. Its reliability depends on the stability of the correlations with the external observables. In this very immediate sense decoher- 
ence enforces the apparent "collapse of the wavepacket": After a decoherence timescale, only the einselected memory states will exist and retain useful correlations (Zurek, 1991; 1998a,b; Tegmark, 2000). The observer described by some specific einselected state (including a configuration of memory bits) will be able to access ("recall") only that state. The collapse is a consequence of einselection and of the one-to-one correspondence between the state of his memory and of the information encoded in it. Memory is simultaneously a description of the recorded information and a part of the "identity tag", defining observer as a physical system. It is as inconsistent to imagine observer perceiving something else than what is implied by the stable (einselected) records in his possession as it is impossible to imagine the same person with a different DNA: Both cases involve information encoded in a state of a system inextricably linked with the physical identity of an individual.

Distinct memory/identity states of the observer (that are also his "states of knowledge") cannot be superposed: This censorship is strictly enforced by decoherence and the resulting einselection. Distinct memory states label and "inhabit" different branches of the Everett's "Many Worlds" Universe. Persistence of correlations is all that is needed to recover "familiar reality". In this manner, the distinction between epistemology and ontology is washed away: To put it succinctly (Zurek, 1994) there can be no information without representation in physical states.

There is usually no need to trace the collapse all the way to observer's memory. It suffices that the states of a decohering system quickly evolve into mixtures of the preferred (pointer) states. All that can be known in principle about a system (or about an observer, also introspectively, e.g., by the observer himself) is its decoherence-resistant 'identity tag' - a description of its einselected state.

Apart from this essentially negative function of a censor the environment plays also a very different role of a "broadcasting agent", relentlessly cloning the information about the einselected pointer states. This role of the environment as a witness in determining what exists was not appreciated until now: Throughout the past two decades, study of decoherence focused on the effect of the environment on the system. This has led to a multitude of technical advances we shall review, but it has also missed one crucial point of paramount conceptual importance: Observers monitor systems indirectly, by intercepting small fractions of their environments (e.g., a fraction of the photons that have been reflected or emitted by the object of interest). Thus, if the understanding of why we perceive quantum Universe as classical is the principal aim, study of the nature of accessibility of information spread throughout the environment should be the focus of attention. This leads one away from the models of measurement inspired by the "von Neumann chain" (1932) to studies of information transfer involving branching out conditional dynamics and the resulting "fan-out" of the information throughout envi- ronment (Zurek, 1983, 1998a, 2000). This new 'quantum Darwinism' view of environment selectively amplifying einselected pointer observables of the systems of interest is complementary to the usual image of the environment as the source of perturbations that destroy quantum coherence of the system. It suggests the redundancy of the imprint of the system in the environment may be a quantitative measure of relative objectivity and hence of classicality of quantum states. It is introduced in Sections VI and VII of this review.

Benefits of recognition of the role of environment include not just operational definition of the objective existence of the einselected states, but - as is also detailed in Section VI - a clarification of the connection between the quantum amplitudes and probabilities. Einselection converts arbitrary states into mixtures of well defined possibilities. Phases are envariant: Appreciation of envariance as a symmetry tied to the ignorance about the state of the system was the missing ingredient in the attempts of 'no collapse' derivations of Born's rule and in the probability interpretation. While both envariance and the "environment as a witness" point of view are only beginning to be investigated, the extension of the program of einselection they offer allowes one to understand emergence of "classical reality" form the quantum substrate as a consequence of quantum laws.

\section{QUANTUM MEASUREMENTS}

The need for a transition from quantum determinism of the global state vector to classical definiteness of states of individual systems is traditionally illustrated by the example of quantum measurements. An outcome of a "generic" measurement of the state of a quantum system is not deterministic. In the textbook discussions this random element is blamed on the "collapse of the wavepacket", invoked whenever a quantum system comes into contact with a classical apparatus. In a fully quantum discussion this issue still arises, in spite (or rather because) of the overall deterministic quantum evolution of the state vector of the Universe: As pointed out by von Neumann (1932), there is no room for a 'real collapse' in the purely unitary models of measurements.

\section{A. Quantum conditional dynamics}

To illustrate the ensuing difficulties, consider a quantum system $\mathcal{S}$ initially in a state $|\psi\rangle$ interacting with a quantum apparatus $\mathcal{A}$ initially in a state $\left|A_{0}\right\rangle$ :

$$
\begin{array}{r}
\left|\Psi_{0}\right\rangle=|\psi\rangle\left|A_{0}\right\rangle=\left(\sum_{i} a_{i}\left|s_{i}\right\rangle\right)\left|A_{0}\right\rangle \\
\longrightarrow \sum_{i} a_{i}\left|s_{i}\right\rangle\left|A_{i}\right\rangle=\left|\Psi_{t}\right\rangle .
\end{array}
$$

Above, $\left\{\left|A_{i}\right\rangle\right\}$ and $\left\{\left|s_{i}\right\rangle\right\}$ are states in the Hilbert spaces of the apparatus and of the system, respectively, and $a_{i}$ 
are complex coefficients. Conditional dynamics of such premeasurement (as the step achieved by Eq. (2.1) is often called) can be accomplished by means of a unitary Schrödinger evolution. Yet it is not enough to claim that a measurement has been achieved: Equation (2.1) leads to an uncomfortable conclusion: $\left|\Psi_{t}\right\rangle$ is an EPR-like entangled state. Operationally, this EPR nature of the state emerging from the premeasurement can be made more explicit by re-writing the sum in a different basis:

$$
\left|\Psi_{t}\right\rangle=\sum_{i} a_{i}\left|s_{i}\right\rangle\left|A_{i}\right\rangle=\sum_{i} b_{i}\left|r_{i}\right\rangle\left|B_{i}\right\rangle .
$$

This freedom of basis choice - basis ambiguity - is guaranteed by the principle of superposition. Therefore, if one were to associate states of the apparatus (or the observer) with decompositions of $\left|\Psi_{t}\right\rangle$, then even before enquiring about the specific outcome of the measurement one would have to decide on the decomposition of $\left|\Psi_{t}\right\rangle$; the change of the basis redefines the measured quantity.

\section{Controlled not and a bit-by-bit measurement}

The interaction required to entangle the measured system and the apparatus, Eq. (2.1), is a generalization of the basic logical operation known as a "controlled not" or a c-not. Classical, c-not changes the state $a_{t}$ of the target when the control is 1 , and does nothing otherwise:

$$
0_{\mathrm{c}} a_{\mathrm{t}} \longrightarrow 0_{\mathrm{c}} a_{\mathrm{t}} ; \quad 1_{\mathrm{c}} a_{\mathrm{t}} \longrightarrow 1_{\mathrm{c}} \neg a_{\mathrm{t}}
$$

Quantum c-not is a straightforward quantum version of Eq. (2.3). It was known as a "bit by bit measurement" (Zurek, 1981; 1983) and used to elucidate the connection between entanglement and premeasurement already before it acquired its present name and significance in the context of quantum computation (see e.g. Nielsen and Chuang, 2000). Arbitrary superpositions of the control bit and of the target bit states are allowed:

$$
\begin{aligned}
\left(\alpha\left|0_{\mathrm{c}}\right\rangle+\right. & \left.+\beta\left|1_{\mathrm{c}}\right\rangle\right)\left|a_{\mathrm{t}}\right\rangle \\
& \longrightarrow \alpha\left|0_{\mathrm{c}}\right\rangle\left|a_{\mathrm{t}}\right\rangle+\beta\left|1_{\mathrm{c}}\right\rangle\left|\neg a_{\mathrm{t}}\right\rangle
\end{aligned}
$$

Above "negation" $\left|\neg a_{\mathrm{t}}\right\rangle$ of a state is basis dependent;

$$
\neg\left(\gamma\left|0_{\mathrm{t}}\right\rangle+\delta\left|1_{\mathrm{t}}\right\rangle\right)=\gamma\left|1_{\mathrm{t}}\right\rangle+\delta\left|0_{\mathrm{t}}\right\rangle
$$

With $\left|A_{0}\right\rangle=\left|0_{\mathrm{t}}\right\rangle,\left|A_{1}\right\rangle=\left|1_{\mathrm{t}}\right\rangle$ we have an obvious analogy between the c-not and a premeasurement.

In the classical controlled not the direction of information transfer is consistent with the designations of the two bits: The state of the control remains unchanged while it influences the target, Eq. (2.3). Classical measurement need not influence the system. Written in the logical basis $\{|0\rangle,|1\rangle\}$, the truth table of the quantum c-not is essentially - that is, save for the possibility of superpositions - the same as Eq. (2.3). One might have anticipated that the direction of information transfer and the designations ("control/system" and "target/apparatus") of the two qubits will be also unambiguous, as in the classical case. This expectation is incorrect. In the conjugate basis $\{|+\rangle,|-\rangle\}$ defined by:

$$
| \pm\rangle=(|0\rangle \pm|1\rangle) / \sqrt{2},
$$

the truth table of Eq. (2.3) along with Eq. (2.6) lead to a new complementary truth table:

$$
| \pm\rangle|+\rangle \longrightarrow| \pm\rangle|+\rangle ;| \pm\rangle|-\rangle \longrightarrow|\mp\rangle|-\rangle .
$$

In the complementary basis $\{|+\rangle,|-\rangle\}$ roles of the control and of the target are reversed: The former (basis $\{|0\rangle,|1\rangle\})$ target - represented by the second ket above remains unaffected, while the state of the former control (the first ket) is conditionally "flipped".

In the bit-by-bit case the measurement interaction is:

$$
\begin{gathered}
H_{\text {int }}=g|1\rangle\left\langle\left. 1\right|_{\mathcal{S}} \mid-\right\rangle\left\langle-\left.\right|_{\mathcal{A}}=\right. \\
\frac{g}{2}|1\rangle\left\langle\left. 1\right|_{\mathcal{S}} \otimes(\mathbf{1}-(|0\rangle\langle 1|+| 1\rangle\langle 0|))_{\mathcal{A}}\right.
\end{gathered}
$$

Above, $g$ is a coupling constant, and the two operators refer to the system (i.e., to the former control), and to the apparatus pointer (the former target), respectively. It is easy to see that the states $\{|0\rangle,|1\rangle\}_{\mathcal{S}}$ of the system are unaffected by $H_{\text {int }}$, since;

$$
\left[H_{\text {int }}, e_{0}|0\rangle\left\langle\left. 0\right|_{\mathcal{S}}+e_{1} \mid 1\right\rangle\left\langle\left. 1\right|_{\mathcal{S}}\right]=0\right.
$$

The measured (control) observable $\hat{\epsilon}=e_{0}|0\rangle\left\langle 0\left|+e_{1}\right| 1\right\rangle\langle 1|$ is a constant of motion under $H_{\text {int }}$. c-not requires interaction time $t$ such that $g t=\pi / 2$.

The states $\{|+\rangle,|-\rangle\}_{\mathcal{A}}$ of the apparatus encode the information about phase between the logical states. They have exactly the same "immunity":

$$
\left[H_{\text {int }}, f_{+}|+\rangle\left\langle+\left.\right|_{\mathcal{A}}+f_{-} \mid-\right\rangle\left\langle-\left.\right|_{\mathcal{A}}\right]=0\right.
$$

Hence, when the apparatus is prepared in a definite phase state (rather than in a definite pointer/logical state), it will pass on its phase onto the system, as Eqs. (2.7) (2.8), show. Indeed, $H_{\text {int }}$ can be written as:

$$
\begin{gathered}
H_{\text {int }}=g|1\rangle\left\langle\left. 1\right|_{\mathcal{S}} \mid-\right\rangle\left\langle-\left.\right|_{\mathcal{A}}\right. \\
=\frac{g}{2}(\mathbf{1}-(|-\rangle\langle+|+|+\rangle\langle-|))_{\mathcal{S}} \otimes|-\rangle\left\langle-\left.\right|_{\mathcal{A}}\right.
\end{gathered}
$$

making this "immunity" obvious.

This basis-dependent direction of information flow in a quantum c-not (or in a premeasurement) is a consequence of complementarity. While the information about the observable with the eigenstates $\{|0\rangle,|1\rangle\}$ travels from the system to the apparatus, in the complementary $\{|+\rangle,|-\rangle\}$ basis it seems that the apparatus is measured by the system. This remark (Zurek 1998a, b; see also Beckman, Gottesman, and Nielsen, 2001) clarifies the sense in which phases are inevitably "disturbed" in measurements. They are not really destroyed, but, rather, as the apparatus measures a certain observable of the system, the system simultaneously "measures" 
phases between the possible outcome states of the apparatus. This leads to loss of phase coherence: Phases become "shared property" as we shall see in more detail in the discussion of envariance.

The question "what measures what?" (decided by the direction of the information flow) depends on the initial states. In "the classical practice" this ambiguity does not arise. Einselection limits the set of possible states of the apparatus to a small subset.

\section{Measurements and controlled shifts.}

The truth table of a whole class of c-not like transformations that includes general premeasurement, Eq. (2.1), can be written as:

$$
\left|s_{j}\right\rangle\left|A_{k}\right\rangle \longrightarrow\left|s_{j}\right\rangle\left|A_{k+j}\right\rangle
$$

Equation (2.1) follows when $k=0$. One can therefore model measurements as controlled shifts - c-shifts generalizations of the c-not. In the bases $\left\{\left|s_{j}\right\rangle\right\}$ and $\left\{\left|A_{k}\right\rangle\right\}$, the direction of the information flow appears to be unambiguous - from the system $\mathcal{S}$ to the apparatus $\mathcal{A}$. However, a complementary basis can be readily defined (Ivanovic, 1981; Wootters and Fields, 1989);

$$
\left|B_{k}\right\rangle=N^{-\frac{1}{2}} \sum_{l=0}^{N-1} \exp \left(\frac{2 \pi i}{N} k l\right)\left|A_{l}\right\rangle
$$

Above $N$ is the dimensionality of the Hilbert space. Analogous transformation can be carried out on the basis $\left\{\left|s_{i}\right\rangle\right\}$ of the system, yielding states $\left\{\left|r_{j}\right\rangle\right\}$.

Orthogonality of $\left\{\left|A_{k}\right\rangle\right\}$ implies:

$$
\begin{gathered}
\left\langle B_{l} \mid B_{m}\right\rangle=\delta_{l m} . \\
\left|A_{k}\right\rangle=N^{-\frac{1}{2}} \sum_{l=0}^{N-1} \exp \left(-\frac{2 \pi i}{N} k l\right)\left|B_{l}\right\rangle
\end{gathered}
$$

inverts of the transformtion of Eq. (2.14a). Hence:

$$
|\psi\rangle=\sum_{l} \alpha_{l}\left|A_{l}\right\rangle=\sum_{k} \beta_{k}\left|B_{k}\right\rangle
$$

where the coefficients $\beta_{k}$ are:

$$
\beta_{k}=N^{-\frac{1}{2}} \sum_{l=0}^{N-1} \exp \left(-\frac{2 \pi i}{N} k l\right) \alpha_{l} .
$$

Hadamard transform of Eq. (2.6) is a special case of the more general transformation considered here.

To implement the truth tables involved in premeasurements we define observable $\hat{A}$ and its conjugate:

$$
\hat{A}=\sum_{k=0}^{N-1} k\left|A_{k}\right\rangle\left\langle A_{k}\left|; \hat{B}=\sum_{l=0}^{N-1} l\right| B_{l}\right\rangle\left\langle B_{l}\right| . \quad(2.18 a, b)
$$

The interaction Hamiltonian:

$$
H_{i n t}=g \hat{s} \hat{B}
$$

is an obvious generalization of Eqs. (2.9) and (2.12), with $g$ the coupling strength and $\hat{s}$ :

$$
\hat{s}=\sum_{l=0}^{N-1} l\left|s_{l}\right\rangle\left\langle s_{l}\right|
$$

In the $\left\{\left|A_{k}\right\rangle\right\}$ basis $\hat{B}$ is a shift operator,

$$
\hat{B}=\frac{i N}{2 \pi} \frac{\partial}{\partial \hat{A}} .
$$

To show how $H_{\text {int }}$ works, we compute:

$$
\begin{gathered}
\exp \left(-i H_{i n t} t / \hbar\right)\left|s_{j}\right\rangle\left|A_{k}\right\rangle= \\
\left|s_{j}\right\rangle N^{-\frac{1}{2}} \sum_{l=0}^{N-1} \exp [-i(j g t / \hbar+2 \pi k / N) l]\left|B_{l}\right\rangle
\end{gathered}
$$

We now adjust the coupling $g$ and the duration of the interaction $t$ so that the action $\iota$ expressed in Planck units $2 \pi \hbar$ is a multiple of $1 / N$;

$$
\iota=g t / \hbar=G * 2 \pi / N
$$

For an integer $G$, Eq. (2.22) can be readily evaluated:

$$
\exp \left(-i H_{i n t} t / \hbar\right)\left|s_{j}\right\rangle\left|A_{k}\right\rangle=\left|s_{j}\right\rangle\left|A_{\{k+G * j\}_{N}}\right\rangle .
$$

This is a shift of the apparatus state by an amount $G * j$ proportional to the eigenvalue $j$ of the state of the system. $G$ plays the role of gain. The index $\{k+G * j\}_{N}$ is evaluated modulo $N$, where $N$ is the number of the possible outcomes, that is, the dimensionality of the Hilbert space of the apparatus pointer $\mathcal{A}$ : When $G * j>N$, the pointer will just rotate through the initial "zero". The truth table for $G=1$ defines a c-shift, Eq. (2.13), and with $k=0$ leads to a premeasurement, Eq. (2.1).

The form of the interaction, Eq. (2.19), in conjunction with the initial state decide the direction of information transfer. Note that - as was the case with the c-nots - the observable that commutes with the interaction Hamiltonian will not be perturbed:

$$
\left[H_{\text {int }}, \hat{s}\right]=0
$$

$\hat{s}$ commutes with $H_{\text {int }}$, and is therefore a non-demolition observable (Braginsky, Vorontsov and Thorne, 1980; Caves et al, 1980; Bocko and Onofrio, 1996).

\section{Amplification}

Amplification was often regarded as the process forcing quantum potentialities to become classical reality. Its example is an extension of the model of measurement described above. 
Assume the Hilbert space of the apparatus pointer is large compared with the space spanned by the eigenstates of the measured observable $\hat{s}$ :

$$
N=\operatorname{Dim}\left(\mathcal{H}_{\mathcal{A}}\right) \gg \operatorname{Dim}\left(\mathcal{H}_{\mathcal{S}}\right)=n
$$

Then one can increase $\iota$ to an integer multiple $G$ of $2 \pi / N$. This was implicit in Eqs. (2.23a) and (2.24). However, larger $\iota$ will lead to redundancy only when the apparatus the Hilbert space has many more dimensions in then there are possible outcomes. Otherwise, only "wrapping" of the same record will ensue. The simplest example of such wrapping: $(\mathrm{c}-\text { not })^{2}$ is the identity. For $N \gg n$, however, one can attain gain:

$$
G=N g t / 2 \pi \hbar \text {. }
$$

The outcomes are now separated by $G-1$ "empty" eigenstates of the record observable. In this sense $G \gg 1$ achieves redundancy, providing that wrapping of the record is avoided, which is guaranteed when:

$$
n G<N \text {. }
$$

Amplification is useful in presence of noise. For example, it may be difficult to initiate the apparatus in $\left|A_{0}\right\rangle$, so the initial state may be a superposition;

$$
\left|a_{l}\right\rangle=\sum_{k} \alpha_{l}(k)\left|A_{k}\right\rangle .
$$

Indeed, typically a mixture of such superpositions;

$$
\rho_{\mathcal{A}}^{0}=\sum_{i} w_{i}\left|a_{i}\right\rangle\left\langle a_{i}\right|
$$

may be the starting point for a premeasurement. Then:

$$
\begin{array}{r}
\left|s_{k}\right\rangle\left\langle s_{k^{\prime}}\left|\rho_{\mathcal{A}}=\right| s_{k}\right\rangle\left\langle s_{k^{\prime}}\left|\sum_{l} w_{l}\right| a_{l}\right\rangle\left\langle a_{l}\right| \\
\longrightarrow\left|s_{k}\right\rangle\left\langle s_{k^{\prime}}\left|\sum_{l} w_{l}\right| a_{l+G k}\right\rangle\left\langle a_{l+G k^{\prime}}\right|
\end{array}
$$

where $\left|a_{l+G k}\right\rangle$ obtains from $\left|a_{l}\right\rangle$, Eq. (2.28a), through:

$$
\left|a_{l+G k}\right\rangle=\sum_{j} \alpha_{l}(j)\left|A_{j+G k}\right\rangle,
$$

and the simplifying assumption about the coefficients;

$$
\alpha_{l}(j)=\alpha(j-l)
$$

has been made. Its aim to focus on the case when the apparatus states are peaked around a certain value $l$ (e.g., $\alpha_{l}(j) \sim \exp \left(-(j-l)^{2} / 2 \Delta^{2}\right)$, and where the form of their distribution over $\left\{\left|A_{k}\right\rangle\right\}$ does not depend on $l$.

A good measurement allows one to distinguish states of the system. Hence, it must satisfy:

$$
\begin{gathered}
\left|\left\langle a_{l+G k} \mid a_{l+G k^{\prime}}\right\rangle\right|^{2} \\
=\left|\sum_{j} \alpha\left(j+G\left(k-k^{\prime}\right)\right) \alpha^{*}(j)\right|^{2} \approx \delta_{k^{\prime}, k} .
\end{gathered}
$$

States of the system that need to be distinguished should rotate the pointer of the apparatus to the correlated outcome states that are approximately orthogonal. When the coefficients $\alpha(k)$ are peaked around $k=0$ with dispersion $\Delta$, this implies:

$$
\Delta \ll G .
$$

In the general case of an initial mixture, Eq. (2.29), one can evaluate the dispersion of the expectation value of the record observable $\hat{A}$ as:

$$
\left\langle\hat{A}^{2}\right\rangle-\langle\hat{A}\rangle^{2}=\operatorname{Tr} \rho_{\mathcal{A}}^{0} \hat{A}^{2}-\left(\operatorname{Tr} \rho_{\mathcal{A}}^{0} \hat{A}\right)^{2}
$$

The outcomes are distinguishable when:

$$
\left\langle\hat{A}^{2}\right\rangle-\langle\hat{A}\rangle^{2} \ll G
$$

Interaction with the environment yields a mixture of the form of Eq. (2.29). Amplification can protect measurement outcomes from noise through redundancy. ${ }^{3}$

3 The above model of amplification is unitary. Yet, it contains seeds of irreversibility. Reversibility of c-shift is evident: As the interaction continues, the two systems will eventually disentangle. For instance, it takes $t_{e}=2 \pi \hbar /(g N)$ (see Eq. (2.23b) with $G=1)$ to entangle $\mathcal{S}\left(\operatorname{Dim}\left(\mathcal{H}_{\mathcal{S}}\right)=n\right)$ with an $\mathcal{A}$ with $\operatorname{Dim}\left(\mathcal{H}_{\mathcal{A}}\right)=N \geq n$ pointer states. However, as the interaction continues, $\mathcal{A}$ and $\mathcal{S}$ disentangle. For a c-shift this recurrence timescale is: $t_{R e c}=N t_{e}=2 \pi \hbar / g$. It corresponds to gain $G=N$. Thus, for an instant of less than $t_{e}$ at $t=t_{R e c}$ the apparatus disentangles from the system, as $\{k+N * j\}_{N}=k$. Reversibility results in recurrences of the initial state, but for $N \gg 1$, they are rare.

For less regular interactions (e.g, involving environment) recurrence time is much longer. In that case, $t_{R e c}$ is, in effect, a Poincaré time: $t_{R e c} \sim t_{\text {Poincaré }} \approx N ! t_{e}$. In any case $t_{R e c} \gg t_{e}$ for large $N$. Undoing entanglement in this manner would be exceedingly difficult because one would need to know precisely when to look, and because one would need to isolate the apparatus or the immediate environment from other degrees of freedom - their environments.

The price of letting the entanglement undo itself by waiting for an appropriate time interval is at the very least given by the cost of storing the information on how long is it necessary to wait. In the special c-shift case this is $\sim \log N$ memory bits. In situations when eigenvalues of the interaction Hamiltonian are not commensurate, it will be more like $\sim \log N ! \approx N \log N$, as the entanglement will get undone only after a Poincaré time. Both classical and quantum case can be analyzed using algorithmic information . For related discussions see Zurek, (1989), Caves, (1994); Schack and Caves (1996) and Zurek (1998b).

Amplified correlations are hard to contain. The return to purity after $t_{\text {Rec }}$ in the manner described above can be hoped for only when either the apparatus or the immediate environment $\mathcal{E}$ (i.e., the environment directly interacting with the system) cannot "pass on" the information to their more remote environments $\mathcal{E}^{\prime}$. The degree of isolation required puts a stringent limit on the coupling $g_{\mathcal{E} \mathcal{E}^{\prime}}$ between the two environments: Return to purity can be accomplished in this manner only if $t_{\text {Rec }}<t_{e^{\prime}}=2 \pi \hbar /\left(N^{\prime} g_{\mathcal{E} \mathcal{E}^{\prime}}\right)$, where $N^{\prime}$ is the dimension of the Hilbert space of the environment $\mathcal{E}^{\prime}$. Hence, the two estimates of $t_{\text {Rec }}$ translate into: $g_{\mathcal{E} \mathcal{E}^{\prime}}<g / N^{\prime}$ for the regular spectrum, and the much tighter; $g_{\mathcal{E} \mathcal{E}^{\prime}}<g / N ! N^{\prime}$ for the random case more 


\section{B. Information transfer in measurements}

Information transfer is the objective of the measurement process. Yet, quantum measurements were only rarely analyzed from that point of view. As a result of the interaction of the system $\mathcal{S}$ with the apparatus $\mathcal{A}$, their joint state is still pure $\left|\Psi_{t}\right\rangle$, Eq. (2.1), but each of the subsystems is in a mixture:

$$
\begin{aligned}
\rho_{\mathcal{S}}=\operatorname{Tr}_{\mathcal{A}}\left|\Psi_{t}\right\rangle\left\langle\Psi_{t}\right| & =\sum_{i=0}^{N-1}\left|a_{i}\right|^{2}\left|s_{i}\right\rangle\left\langle s_{i}\right| ; \\
\rho_{\mathcal{A}}=\operatorname{Tr}_{\mathcal{S}}\left|\Psi_{t}\right\rangle\left\langle\Psi_{t}\right| & =\sum_{i=0}^{N-1}\left|a_{i}\right|^{2}\left|A_{i}\right\rangle\left\langle A_{i}\right| .
\end{aligned}
$$

Partial trace leads to reduced density matrices, here $\rho_{\mathcal{S}}$ and $\rho_{\mathcal{A}}$, important for what follows. They describe subsystems to the observer who, before the premeasurement, knew pure states of the system and of the apparatus, but who has access to only one of them afterwards.

Reduced density matrix is a technical tool of paramount importance. It was introduced by Landau (1927) as the only density matrix that gives rise to the correct measurement statistics given the usual formalism that includes Born's rule for calculating probabilities (see e.g. p. 107 of Nielsen and Chuang, 2000, for an insightful discussion). This remark will come to haunt us later when in Section VI we shall consider the relation between decoherence and probabilities: In order to derive Born's rule it will be important not to assume it in some guise!

Following premeasurement, the information about the subsystems available to the observer locally decreases. This is quantified by the increase of the entropies:

$$
\begin{aligned}
H_{\mathcal{S}} & =-\operatorname{Tr} \rho_{\mathcal{S}} \log \rho_{\mathcal{S}}=-\sum_{i=0}^{N-1}\left|a_{i}\right|^{2} \log \left|a_{i}\right|^{2} \\
& =-\operatorname{Tr} \rho_{\mathcal{A}} \log \rho_{\mathcal{A}}=H_{\mathcal{A}}
\end{aligned}
$$

As the evolution of the whole $\mathcal{S} \mathcal{A}$ is unitary, the increase of entropies in the subsystems is compensated by the increase of the mutual information:

$$
\mathcal{I}(\mathcal{S}: \mathcal{A})=H_{\mathcal{S}}+H_{\mathcal{A}}-H_{\mathcal{S} \mathcal{A}}=-2 \sum_{i=0}^{N-1}\left|a_{i}\right|^{2} \log \left|a_{i}\right|^{2}(14)
$$

It was used in quantum theory as a measure of entanglement (Zurek, 1983; Barnett and Phoenix, 1989).

relevant for decoherence.

In short, once information "leaks" into the correlations between the system and the apparatus or the environment, keeping it from spreading further ranges between very hard and next to impossible. With the exception of very special cases (small $N$, regular spectrum), the strategy of "enlarging the system, so that it includes the environment" - occasionally mentioned as an argument against decoherence - is doomed to fail, unless the Universe as a whole is included. This is a questionable setting (as the observers are inside this "isolated" system) and in any case makes the relevant Poincaré time absurdly long!

\section{Action per bit}

An often raised question concerns the price of information in units of some other "physical currency", (Brillouin, 1962; 1964; Landauer, 1991). Here we shall establish that the least action necessary to transfer one bit is of the order of a fraction of $\hbar$ for quantum systems with two-dimensional Hilbert spaces. Information transfer can be made cheaper on the "wholesale" level, when the systems involved have large Hilbert spaces.

Consider Eq. (2.1). It evolves initial product state of the two subsystems into a superposition of product states, $\left(\sum_{j} \alpha_{j}\left|s_{j}\right\rangle\right)\left|A_{0}\right\rangle \longrightarrow \sum_{j} \alpha_{j}\left|s_{j}\right\rangle\left|A_{j}\right\rangle$. The expectation value of the action involved is no less than:

$$
I=\sum_{j=0}^{N-1}\left|\alpha_{j}\right|^{2} \arccos \left|\left\langle A_{0} \mid A_{j}\right\rangle\right|
$$

When $\left\{\left|A_{j}\right\rangle\right\}$ are mutually orthogonal, the action is:

$$
I=\pi / 2
$$

in Planck $(\hbar)$ units. This estimate can be lowered by using the initial $\left|A_{0}\right\rangle$, a superposition of the outcomes $\left|A_{j}\right\rangle$. In general, interaction of the form:

$$
H_{\mathcal{S A}}=i g \sum_{k=0}^{N-1}\left|s_{k}\right\rangle\left\langle s_{k}\right| \sum_{l=0}^{N-1}\left(\left|A_{k}\right\rangle\left\langle A_{l}\right|-\text { h.c. }\right)
$$

saturates the lower bound given by:

$$
I=\arcsin \sqrt{1-1 / N}
$$

For a two-dimensional Hilbert space the average action can be thus brought down to $\pi \hbar / 4$ (Zurek, 1981; 1983).

As the size of the Hilbert space increases, action involved approaches the asymptotic estimate of Eq. (2.40). The entropy of entanglement can be as large as $\log N$ where $N$ is the dimension of the Hilbert space of the smaller of the two systems. Thus, the least action per bit of information decreases with the increase of $N$ :

$$
\iota=\frac{I}{\log _{2} N} \approx \frac{\pi}{2 \log _{2} N}
$$

This may be one reason why information appears "free" in the macroscopic domain, but expensive (close to $\hbar /$ bit) in the quantum case of small Hilbert spaces.

\section{C. "Collapse" analogue in a classical measurement}

Definite outcomes we perceive appear to be at odds with the principle of superposition. They can nevertheless occur also in quantum physics when the initial state of the measured system is - already before the measurement - in one of the eigenstates of the measured observable. Then Eq. (2.1) will deterministically rotate the pointer of the apparatus to the appropriate record state. 
The result of such a measurement can be predicted by an insider - an observer aware of the initial state of the system. This a priori knowledge can be represented by the preexisting record $\left|A_{i}\right\rangle$, which is only corroborated by an additional measurement:

$$
\left|A_{i}\right\rangle\left|A_{0}\right\rangle\left|\sigma_{i}\right\rangle \longrightarrow\left|A_{i}\right\rangle\left|A_{i}\right\rangle\left|\sigma_{i}\right\rangle
$$

In classical physics complete information about the initial state of an isolated system always allows for an exact prediction of its future state: A well-informed observer will be even able to predict future of the classical Universe as a whole ("Laplace's demon").

Any element of surprise (any use of probabilities) must be therefore blamed on partial ignorance. Thus, when the information available initially does not include the exact initial state of the system, observer can use an ensemble described by $\rho_{\mathcal{S}}$ - by a list of possible initial states $\left\{\left|\sigma_{i}\right\rangle\right\}$ and their probabilities $p_{i}$. This is the ignorance interpretation of probabilities. We shall see in section VI that - using quantum envariance - one can justify ignorance about a part of the system by relying on perfect knowledge of the whole.

Through measurement observer finds out which of the potential outcomes consistent with his prior (lack of) information actually happens. This act of acquisition of information changes physical state of the observer - the state of his memory: The initial memory state containing description $A_{\rho_{S}}$ of an ensemble and a "blank" $A_{0}$, $\left|A_{\rho_{\mathcal{S}}}\right\rangle\left\langle A_{\rho_{\mathcal{S}}}|| A_{0}\right\rangle\left\langle A_{0}\right|$, is transformed into record of a specific outcome: $\left|A_{\rho_{\mathcal{S}}}\right\rangle\left\langle A_{\rho_{\mathcal{S}}}|| A_{i}\right\rangle\left\langle A_{i}\right|$. In quantum notation this process will be described by such a discoverer as a random "collapse":

$$
\begin{gathered}
\left|A_{\rho_{\mathcal{S}}}\right\rangle\left\langle A_{\rho_{\mathcal{S}}}|| A_{0}\right\rangle\left\langle A_{0}\left|\sum_{i} p_{i}\right| \sigma_{i}\right\rangle\left\langle\sigma_{i}\right| \\
\longrightarrow\left|A_{\rho_{\mathcal{S}}}\right\rangle\left\langle A_{\rho_{\mathcal{S}}}|| A_{i}\right\rangle\left\langle A_{i}|| \sigma_{i}\right\rangle\left\langle\sigma_{i}\right| .
\end{gathered}
$$

This is only the description of what happens "as reported by the discoverer". Deterministic representation of this very same process by Eq. (2.44a) is still possible. In other words, in classical physics discoverer can be always convinced that the system was in a state $\left|\sigma_{i}\right\rangle$ already before the record is made in accord with Eq. (2.44b).

This sequence of events as seen by the discoverer looks like a "collapse" (see also Zurek, 1998a,b): For instance, insider who knew the state of the system before discoverer carried out his measurement need not notice any change of that state when he makes further "confirmatory" measurements. This property is the cornerstone of the "reality" of classical states - they need not ever change as a consequence of measurements. We emphasize, however, that while the state of the system may remain unchanged, the state of the observer must change to reflect the acquired information.

Last not least, an outsider-someone who knows about the measurement, but (in contrast to the insider) not about the initial state of the system nor (in contrast to the discoverer) about the measurement outcome, will describe the same process still differently:

$$
\begin{aligned}
& \left|A_{\rho_{\mathcal{S}}}\right\rangle\left\langle A_{\rho_{\mathcal{S}}}|| A_{0}\right\rangle\left\langle A_{0}\left|\sum_{i} p_{i}\right| \sigma_{i}\right\rangle\left\langle\sigma_{i}\right| \longrightarrow \\
& \longrightarrow\left|A_{\rho_{\mathcal{S}}}\right\rangle\left\langle A_{\rho_{\mathcal{S}}}\right|\left(\sum_{i} p_{i}\left|A_{i}\right\rangle\left\langle A_{i}|| \sigma_{i}\right\rangle\left\langle\sigma_{i}\right|\right)
\end{aligned}
$$

This view of the outsider, Eq. (2.44c), combines one-toone classical correlation of the states of the system and the records with the indefiniteness of the outcome.

We have just seen three distinct quantum-looking descriptions of the very same classical process (see Zurek, 1989, and Caves, 1994 for previous studies of the insider - outsider theme). They differ only in the information available $a b$ initio to the observer. The information in possession of the observer prior to the measurement determines in turn whether - to the observer - the evolution appears to be (a) a confirmation of the preexisting data, Eq. (2.44a), (b) a "collapse" associated with the information gain, Eq. (2.44b) - and with the entropy decrease translated into algorithmic randomness of the acquired data (Zurek, 1989; 1998b) - or (c) an entropy-preserving establishment of a correlation, Eq. (2.44c). All three descriptions are classically compatible, and can be implemented by the same (deterministic and reversible) dynamics.

In classical physics the insider view always exists in principle. In quantum physics it does not. Every observer in a classical Universe could in principle aspire to be an ultimate insider. The fundamental contradiction between every observer knowing precisely the state of the rest of the Universe (including the other observers) can be swept under the rug (if not really resolved) in the Universe where the states are infinitely precisely determined and the observer's records (as a consequence of the $\hbar \rightarrow 0$ limit) may have an infinite capacity for information storage. However - given a set value of $\hbar$ - information storage resources of any finite physical system are finite. Hence, in quantum physics observers remain largely ignorant of the detailed state of the Universe as there can be no information without representation (Zurek, 1994).

Classical "collapse" is described by Eq. (2.44b): The observer discovers the state of the system. From then on, the state of the system will remain correlated with his record, so that all the future outcomes can be predicted, in effect by iterating Eq. (2.44a). This disappearance of all the potential alternatives save for one that becomes a "reality" is the essence of the collapse. There need not be anything quantum about it.

Einselection in observers memory provides many of the ingredients of the "classical collapse" in the quantum context. In presence of einselection, one-to-one correspondence between the state of the observer and his knowledge about the rest of the Universe can be firmly established, and (at least in principle) operationally verified: One could measure bits in observers memory and determine what he knows without altering his records - 
without altering his state. After all, one can do so with a classical computer. Existential interpretation recognizes that the information possessed by the observer is reflected in his einselected state, explaining his perception of a single "branch" - "his" classical Universe.

\section{CHAOS AND LOSS OF CORRESPONDENCE}

The study of the relationship between the quantum and the classical has been - for a long time - focused almost entirely on measurements. However, the problem of measurement is difficult to discuss without observers. And once observer enters, it is often hard to avoid its illunderstood anthropic attributes such as consciousness, awareness, and the ability to perceive.

We shall sidestep these presently "metaphysical" problems and focus on the information-processing underpinnings of the "observership". It is nevertheless fortunate that there is another problem with the quantum - classical correspondence that leads to interesting questions not motivated by measurements. As was anticipated by Einstein (1917) before the advent of modern quantum theory, chaotic motion presents such a challenge. Full implications of classical dynamical chaos were understood much later. The concern about the quantum-classical correspondence in this modern context dates to Berman and Zaslavsky (1978) and Berry and Balazs (1979) (see Haake, 1991 and Casati and Chirikov, 1995a, for references). It has even led some to question validity of quantum theory (Ford and Mantica, 1992).

\section{A. Loss of the quantum-classical correspondence}

The interplay between quantum interference and chaotic exponential instability leads to the rapid loss of the quantum-classical correspondence. Chaos in dynamics is characterized by the exponential divergence of the classical trajectories. As a consequence, a small patch representing the probability density in phase space is exponentially stretching in unstable directions and to exponentially compressing in the stable directions. The rates of stretching and compression are given by positive and negative Lyapunov exponents $\Lambda_{i}$. Hamiltonian evolution demands that the sum of all the Lyapunov exponents be zero. In fact, they appear in $\pm \Lambda_{i}$ pairs.

Loss of the correspondence in chaotic systems is a consequence of the exponential stretching of the effective support of the probability distribution in the unstable direction (say, $x$ ) and its exponential narrowing in the complementary direction (Zurek and Paz, 1994; Zurek, 1998b). As a consequence, classical probability distribution will develop structures on the scale:

$$
\Delta p \sim \Delta p_{0} \exp (-\Lambda t)
$$

Above, $\Delta p_{0}$ is the measure of the initial momentum spread. $\Lambda$ is the net rate of contraction in the direction of momentum given by the Lyapunov exponents (but see Boccaletti, Farini, and Arecchi, 1997). In the real chaotic system stretching and narrowing of the probability distribution in both $x$ and $p$ occur simultaneously, as the initial patch is rotated and folded. Eventually, the envelope of its effective support will swell to fill in the available phase space, resulting in the wavepacket coherently spread over the spatial region of no less than;

$$
\Delta x \sim\left(\hbar / \Delta p_{0}\right) \exp (\Lambda t)
$$

until it becomes confined by the potential, while the small-scale structure will continue to descend to ever smaller scales (Fig. 1). Breakdown of the quantumclassical correspondence can be understood in two complementary ways, either as a consequence of small $\Delta p$ (see the discussion of Moyal bracket below), or as a result of large $\Delta x$.

Coherent exponential spreading of the wavepacket large $\Delta x$ - must cause problems with correspondence. This is inevitable, as classical evolution appeals to the idealization of a point in phase space acted upon by a force given by the gradient $\partial_{x} V$ of the potential $V(x)$ evaluated at that point. But quantum wavefunction can be coherent over a region larger than the nonlinearity scale $\chi$ over which the gradient of the potential changes significantly. $\chi$ can be usually estimated by:

$$
\chi \simeq \sqrt{\partial_{x} V / \partial_{x x x} V},
$$

and is typically of the order of the size $L$ of the system:

$$
L \sim \chi
$$

An initially localized state evolving in accord with Eqs. (3.1) and (3.2) will spread over such scales after:

$$
t_{\hbar} \simeq \Lambda^{-1} \ln \frac{\Delta p_{0} \chi}{\hbar}
$$

It is then impossible to tell what is the force acting on the system, as it is not located in any specific $x$. This estimate of what can be thought of as Ehrenfest time - the time over which a quantum system that has started in a localized state will continue to be sufficiently localized for the quantum corrections to the equations of motion obeyed by its expectation values to be negligible (Gottfried, 1966) - is valid for chaotic systems. Logarithmic dependence is the result of inverting of the exponential sensitivity. In the absence of the exponential instability $(\Lambda=0)$ divergence of trajectories is typically polynomial, and leads to a power law dependence, $t_{\hbar} \sim(I / \hbar)^{\alpha}$, where $I$ is the classical action. Thus, macroscopic (large I) integrable systems can follow classical dynamics for a very long time, providing they were initiated in a localized state. For chaotic systems $t_{\hbar}$ also becomes infinite in the limit $\hbar \rightarrow 0$, but that happens only logarithmically slowly. As we shall see below, in the context of quantumclassical correspondence this is too slow for comfort.

Another way of describing the "root cause" of the correspondence breakdown is to note that after the timescale 
of the order of $t_{\hbar}$ quantum wave function of the system would have spread over all of the available space, and is being forced to "fold" onto itself. Fragments of the wavepacket arrive at the same location (although with different momenta, and having followed different paths). The ensuing evolution critically depends on whether they have retained phase coherence. When coherence persists, a complicated interference event decides the subsequent evolution. And - as can be anticipated from the double slit experiment - there is a big difference between coherent and incoherent folding in the configuration space. This translates into the loss of correspondence, which sets in surprisingly quickly, at $t_{\hbar}$.

To find out how quickly, we estimate $t_{\hbar}$ for an obviously macroscopic Hyperion, chaotically tumbling moon of Saturn (Wisdom, 1985). Hyperion has a prolate shape of a potato and moves on an eccentric orbit with a pe$\operatorname{riod} t_{O}=21$ days. Interaction between its gravitational quadrupole and the tidal field of Saturn leads to chaotic tumbling with Lyapunov time $\Lambda^{-1} \simeq 42$ days.

To estimate the time over which orientation of Hyperion becomes delocalized, we use a formula (Berman and Zaslavsky, 1978, Berry and Balazs, 1979):

$$
t_{r}=\Lambda^{-1} \ln \frac{L P}{\hbar}=\Lambda^{-1} \ln \frac{I}{\hbar}
$$

Above $L$ and $P$ give the range of values of the coordinate and momentum in phase space of the system. Since $L \simeq$ $\chi$ and $P>\Delta p_{0}$, it follows that $t_{r} \geq t_{\hbar}$. On the other hand, $L P \simeq I$, the action of the system.

The advantage of Eq. (3.6) is its insensitivity to initial conditions, and the ease with which the estimate can be obtained. For Hyperion, a generous overestimate of the classical action $I$ can be obtained from its binding energy $E_{B}$ and its orbital time $t_{O}$ :

$$
I / \hbar \simeq E_{B} t_{O} / \hbar \simeq 10^{77}
$$

The above estimate (Zurek, 1998b) is "astronomically" large. However, in the calculation of the loss of correspondence, Eq. (3.6), only its logarithm enters. Thus,

$$
t_{r}^{\text {Hyperion }} \simeq 42[\text { days }] \ln 10^{77} \simeq 20[\mathrm{yrs}]
$$

After approximately 20 years Hyperion would be in a coherent superposition of orientations that differ by $2 \pi$ !

We conclude that after a relatively short time an obviously macroscopic chaotic system becomes forced into a flagrantly non-local "Schrödinger cat" state. In the original discussion (Schrödinger, 1935a,b) an intermediate step in which decay products of the nucleus were measured to determine the fate of the cat was essential. Thus, it was possible to maintain that the preposterous superposition of the dead and alive cat could be avoided providing that quantum measurement (with the "collapse" it presumably induces) was properly understood.

This cannot be the resolution for chaotic quantum systems. They can evolve - as the example of Hyperion demonstrates - into states that are non-local, and, therefore, extravagantly quantum, simply as a result of the exponentially unstable dynamics. Moreover, this happens surprisingly quickly, even for very macroscopic examples. Hyperion is not the only chaotic system. There are asteroids that have chaotically unstable orbits (e.g., Chiron), and even indications that the solar system as a whole is chaotic (Laskar, 1989; Sussman and Wisdom, 1992). In all of these cases straightforward estimates of $t_{\hbar}$ yield answers much smaller than the age of the solar system. Thus, if unitary evolution of closed subsystems was a complete description of planetary dynamics, planets would be delocalized along their orbits.

\section{B. Moyal bracket and Liouville flow}

Heuristic argument about breakdown of the quantumclassical correspondence can be made more rigorous with the help of the Wigner function. We start with the von Neumann equation:

$$
i \hbar \dot{\rho}=[H, \rho] .
$$

It can be transformed into the equation for Wigner function $W$, which is defined in phase space:

$$
W(x, p)=\frac{1}{2 \pi \hbar} \int \exp \left(\frac{i p y}{\hbar}\right) \rho\left(x-\frac{y}{2}, x+\frac{y}{2}\right) d y .
$$

The result is:

$$
\dot{W}=\{H, W\}_{M B} .
$$

Here $\{\ldots, \ldots\}_{M B}$ stands for Moyal bracket, the Wigner transform of the von Neumann bracket (Moyal, 1949).

Moyal bracket can be expressed in terms of the Poisson bracket $\{\ldots, \ldots\}$, which generates Liouville flow in the classical phase space, by the formula:

$$
i \hbar\{\ldots, \ldots\}_{M B}=\sin (i \hbar\{\ldots, \ldots\}) .
$$

When the potential $V(x)$ is analytic, Moyal bracket can be expanded (Hillery et al, 1984) in powers of $\hbar$ :

$$
\dot{W}=\{H, W\}+\sum_{n \geq 1} \frac{\hbar^{2 n}(-)^{n}}{2^{2 n}(2 n+1) !} \partial_{x}^{2 n+1} V \partial_{p}^{2 n+1} W .
$$

The first term is just the Poisson bracket. Alone, it would generate classical motion in phase space. However, when the evolution is chaotic, quantum corrections (proportional to the odd order momentum derivatives of the Wigner function) will eventually dominate the right hand side of Eq. (3.10). This is because the exponential squeezing of the initially regular patch in phase space (which begins its evolution in the classical regime, where the Poisson bracket dominates) leads to an exponential explosion of the momentum derivatives. Consequently, after a time logarithmic in $\hbar$, (Eqs. (3.5), (3.6)) the Poisson bracket will cease to be a good estimate of the right hand side of Eq. (3.13). 
The physical reason for the ensuing breakdown of the quantum-classical correspondence was already explained before: Exponential instability of the chaotic evolution delocalizes the wavepacket. As a result, the force acting on the system is no longer given by the gradient of the potential evaluated at the location of the system: It is not even possible to say where the system is, since it is in a superposition of many distinct locations. Consequently, phase space distribution and even the typical observables of the system noticeably differ when evaluated classically and quantum mechanically (Haake, Kuś and Sharf, 1987; Habib, Shizume and Zurek, 1998; Karkuszewski, Zakrzewski, and Zurek, 2002). Moreover, this will happen after an uncomfortably short time $t_{\hbar}$.

\section{Symptoms of correspondence loss}

Wavepacket becomes rapidly delocalized in a chaotic system, and the correspondence between classical and quantum is quickly lost. Schrödinger cat states appear after $t_{\hbar}$ and this is the overarching interpretational as well as physical problem: In the familiar real world we never seem to encounter such smearing of the wavefunction even in the examples of chaotic dynamics it is predicted by quantum theory.

\section{Expectation values}

Measurements often average out fine phase space structures, which may be striking, but experimentally inaccessible symptom of breakdown of the correspondence. Thus, one might hope that when interference patterns in the Wigner function are ignored by looking at the coarsegrained distribution, the quantum results should be in accord with the classical. This would not exorcise the 'chaotic cat' problem. Moreover, breakdown of correspondence can be also seen in the expectation values of quantities that are smooth in phase space.

Trajectories diverge exponentially in a chaotic system. A comparison between expectation values for a single trajectory and for a delocalized quantum state (which is how the Ehrenfest theorem mentioned above is usually stated) would clearly lead to a rapid loss of correspondence. One may object to the use of a single trajectory and argue that both quantum and classical state be prepared and accessed only through measurements that are subject to the Heisenberg indeterminacy. Thus, it should be fair to compare averages over an evolving Wigner function with an initially identical classical probability distribution (Ballentine, Yang, and Zibin, 1994; Haake, Kuś \& Sharf, 1987; Fox and Elston, 1994; Miller, Sarkar and Zarum, 1998). These are shown in Fig. 2 for an example of a driven chaotic system. Clearly, there is reason for concern: Fig. 2 (corroborated by other studies, see e.g. Karkuszewski, Zakrzewski, and Zurek, 2002, for references) demonstrates that not just phase space portrait but also averages diverge at a time $\sim t_{\hbar}$.

In integrable systems rapid breakdown of correspondence may still occur, but only for very special initial conditions. It is due to the local instability in phase space. Indeed, a double - slit experiment is an example of a regular system in which a local instability (splitting of the paths) leads to correspondence loss, but only for judiciously selected initial conditions. Thus, one may dismiss it as a consequence of a rare pathological starting point, and argue that the conditions that lead to discrepancies between classical and quantum behavior exist, but are of measure zero in the classical limit.

In the chaotic case the loss of correspondence is typical. As shown in Fig. 2, it happens after a disturbingly short $t_{\hbar}$ for generic initial conditions. The time at which the quantum and classical expectation values diverge in the example studied here is consistent with the estimates of $t_{\hbar}$, Eq. (3.5), but exhibits a significant scatter. This is not too surprising - exponents characterizing local instability vary noticeably with the location in phase space. Hence, stretching and contraction in phase space will occur at a rate that depends on the specific trajectory. Dependence of its typical magnitude as a function of $\hbar$ is still not clear. Emerson and Ballentine (2001a\&b) study coupled spins and argue that it is of order of $\hbar$, but Fig. 2 suggests it decreases more slowly than that, and that it may be only logarithmic in $\hbar$ (Karkuszewski et al. 2002).

\section{Structure saturation}

Evolution of the Wigner function exhibits rapid buildup of interference fringes. These fringes become progressively smaller, until saturation when the wavepacket is spread over the available phase space. At that time their scales in momentum and position are typically:

$$
d p=\hbar / L ; d x=\hbar / P
$$

where $L(P)$ defines the range of positions (momenta) of the effective support of $W$ in phase space.

Hence, smallest structures in the Wigner function occur (Zurek, 2001) on scales corresponding to action:

$$
a=d x d p=\hbar \times \hbar / L P=\hbar^{2} / I,
$$

where $I \simeq L P$ is the classical action of the system. Action $a \ll \hbar$ for macroscopic $I$.

Sub-Planck structure is a kinematic property of quantum states. It limits their sensitivity to perturbations, and has applications outside quantum chaos or decoherene. For instance, Schrödinger cat state can be used as a weak force detector (Zurek, 2001), and its sensitivity is determined by Eqs. (3.14)-(3.16).

Structure saturation on scale $a$ is an important distinction between quantum and classical. In chaotic systems smallest structures in the classical probability density exponentially shrink with time, in accord with Eq. (3.1) (see Fig. 1). Equation (3.16) has implications for decoherence, as a controls sensitivity of the systems as well 
as environments (Zurek, 2001; Karkuszewski, Jarzynski, and Zurek, 2002): As a result of smallness of $a$, Eq. (3.16), and as anticipated by Peres (1993), quantum systems are more sensitive to perturbations when their classical counterparts are chaotic (see also Jalabert and Pastawski, 2001). But - in contrast to classical chaotic systems - they are not exponentially sensitive to infinitesimally small perturbations: Rather, the smallest perturbations that can effective are set by Eq. (3.16).

Emergence of Schrödinger cats through dynamics is a challenge to quantum - classical correspondence. It is not yet clear to what extent one should be concerned about the discrepancies between quantum and classical averages: The size of this discrepancy may or may not be negligible. But in the original Schrödinger cat problem quantum and classical expectation values (for the survival of the cat) were also in accord. In both cases it is ultimately the state of the cat which is most worrisome.

Note that we have not dealt with dynamical localization (Casati and Chirikov, 1995a). This is because it appears after too long a time $\left(\sim \hbar^{-1}\right)$ to be a primary concern in the macroscopic limit, and is quite sensitive to small perturbations of the potential (Karkuszewski, Zakrzewski, and Zurek, 2002).

\section{ENVIRONMENT - INDUCED SUPERSELECTION}

The principle of superposition applies only when the quantum system is closed. When the system is open, interaction with the environment results in an incessant monitoring of some of its observables. As a result, pure states turn into mixtures that rapidly diagonalize in the einselected states. These pointer states are chosen with the help of the interaction Hamiltonian and are independent of the initial state of the system. Their predictability is key to the effective classicality (Zurek, 1993a; Zurek, Habib, and Paz, 1993).

Environments can be external (such as particles of the air or photons that scatter off, say, the apparatus pointer) or internal (collections of phonons or other internal excitations). Often, environmental degrees of freedom emerge from the split of the original set of degrees of freedom into the "system of interest" which may be a collective observable (e.g., an order parameter in a phase transition), and the "microscopic remainder".

The set of einselected states is called the pointer basis (Zurek, 1981) in recognition of its role in measurements. The criterion for the einselection of states goes well beyond the often repeated characterizations based on the instantaneous eigenstates of the density matrix. What is of the essence is the ability of the einselected states to survive monitoring by the environment. This heuristic criterion can be made rigorous by quantifying predictability of the evolution of the candidate states, or of the associated observables. Einselected states provide optimal initial conditions: They can be employed for the purpose of prediction better than other Hilbert space alternatives - they retain correlations in spite of their immersion in the environment.

Three quantum systems - the measured system $\mathcal{S}$, the apparatus $\mathcal{A}$, and the environment $\mathcal{E}$ - and correlations between them are the subject of our study. In pre-measurements $\mathcal{S}$ and $\mathcal{A}$ interact. Their resulting entanglement transforms into an effectively classical correlation as a result of the interaction between $\mathcal{A}$ and $\mathcal{E}$.

This $\mathcal{S} \mathcal{A} \mathcal{E}$ triangle helps analyze decoherence and study its consequences. By keeping all three corners of this triangle in mind, one can avoid confusion, and maintain focus on the correlations between, e.g., the memory of the observer and the state of the measured system. The evolution from a quantum entanglement to the classical correlation may be the easiest relevant theme to define operationally. In the language of the last part of Section II, we are about to justify the "outsider" point of view, Eq. (2.44c), before considering the "discoverer", Eq. (2.44b) and the issue of the "collapse". In spite of this focus on correlations, we shall often suppress one of the corners of the $\mathcal{S} \mathcal{A} \mathcal{E}$ triangle to simplify notation. All three parts will however play a role in formulating questions and in motivating criteria for classicality.

\section{A. Models of einselection}

The simplest case of a single act of decoherence involves just three one-bit systems (Zurek, 1981; 1983). They are denoted by $\mathcal{S}, \mathcal{A}$, and $\mathcal{E}$ in an obvious reference to their roles. The measurement starts with the interaction of the measured system with the apparatus:

$$
|\uparrow\rangle\left|A_{0}\right\rangle \longrightarrow|\uparrow\rangle\left|A_{1}\right\rangle,|\downarrow\rangle\left|A_{0}\right\rangle \longrightarrow|\downarrow\rangle\left|A_{0}\right\rangle ; \quad(4.1 a, b)
$$

where $\left\langle A_{0} \mid A_{1}\right\rangle=0$. For a general state:

$$
(\alpha|\uparrow\rangle+\beta|\downarrow\rangle)\left|A_{0}\right\rangle \longrightarrow \alpha|\uparrow\rangle\left|A_{1}\right\rangle+\beta|\downarrow\rangle\left|A_{0}\right\rangle=|\Phi\rangle .
$$

These formulae are an example of a c-not like the premeasurement discussed in section 2 .

The basis ambiguity - the ability to re-write $|\Phi\rangle$, Eq. (4.2), in any basis of, say, the system, with the superposition principle guaranteeing existence of the corresponding pure states of the apparatus - disappears when an additional system, $\mathcal{E}$, performs a premeasurement on $\mathcal{A}$ :

$$
\begin{aligned}
& \left(\alpha|\uparrow\rangle\left|A_{1}\right\rangle+\beta|\downarrow\rangle\left|A_{0}\right\rangle\right)\left|\varepsilon_{0}\right\rangle \\
& \longrightarrow \alpha|\uparrow\rangle\left|A_{1}\right\rangle\left|\varepsilon_{1}\right\rangle+\beta|\downarrow\rangle\left|A_{0}\right\rangle\left|\varepsilon_{0}\right\rangle=|\Psi\rangle .
\end{aligned}
$$

A collection of three correlated quantum systems is no longer subject to the basis ambiguity we have pointed out in connection with the EPR-like state $|\Phi\rangle$, Eq. (4.2). This is especially true when states of the environment are correlated with the simple products of the states of the apparatus - system combination (Zurek, 1981; Elby and Bub, 1994). In Eq. (4.3) this can be guaranteed (irrespective of the value of $\alpha$ and $\beta$ ) providing that:

$$
\left\langle\varepsilon_{0} \mid \varepsilon_{1}\right\rangle=0 \text {. }
$$


When this orthogonality condition is satisfied, the state of the $\mathcal{A}-\mathcal{S}$ pair is given by a reduced density matrix:

$$
\begin{gathered}
\rho_{\mathcal{A S}}=\operatorname{Tr}_{\mathcal{E}}|\Psi\rangle\langle\Psi|= \\
|\alpha|^{2}|\uparrow\rangle\left\langle\uparrow|| A_{1}\right\rangle\left\langle\left. A_{1}|+| \beta\right|^{2} \mid \downarrow\right\rangle\left\langle\downarrow \| A_{0}\right\rangle\left\langle A_{0}\right|
\end{gathered}
$$

containing only classical correlations.

If the condition of Eq. (4.4) did not hold - that is, if the orthogonal states of the environment were not correlated with the apparatus in the basis in which the original premeasurement was carried out - then the eigenstates of the reduced density matrix $\rho_{\mathcal{A S}}$ would be sums of products rather than simply products of states of $\mathcal{S}$ and $\mathcal{A}$. Extreme example of this situation is the pre-decoherence density matrix of the pure state:

$$
\begin{aligned}
|\Phi\rangle\langle\Phi| & =|\alpha|^{2}|\uparrow\rangle\left\langle\uparrow \| A_{1}\right\rangle\left\langle A_{1}\left|+\alpha \beta^{*}\right| \uparrow\right\rangle\left\langle\downarrow|| A_{1}\right\rangle\left\langle A_{0}\right| \\
& +\alpha^{*} \beta|\downarrow\rangle\left\langle\uparrow \| A_{0}\right\rangle\left\langle\left. A_{1}|+| \beta\right|^{2} \mid \downarrow\right\rangle\left\langle\downarrow \| A_{0}\right\rangle\left\langle A_{0}(21)\right.
\end{aligned}
$$

Its eigenstate is $|\Phi\rangle$. When expanded, $|\Phi\rangle\langle\Phi|$ contains terms that are off-diagonal when expressed in any of the natural bases consisting of the tensor products of states in the two systems. Their disappearance as a result of tracing over the environment removes the basis ambiguity. Thus, for example, the reduced density matrix $\rho_{\mathcal{A S}}$, Eq. (4.5a), has the same form as the outsider description of the classical measurement, Eq. (2.44c).

In our simple model pointer states are easy to characterize: To leave pointer states untouched, the Hamiltonian of interaction $H_{\mathcal{A E}}$ should have the same structure as for the c-not, Eqs. (2.9) - (2.10): It should be a function of the pointer observable; $\hat{A}=a_{0}\left|A_{0}\right\rangle\left\langle A_{0}\right|+$ $a_{1}\left|A_{1}\right\rangle\left\langle A_{1}\right|$ of the apparatus. Then the states of the environment will bear an imprint of the pointer states $\left\{\left|A_{0}\right\rangle,\left|A_{1}\right\rangle\right\}$. As noted in section II:

$$
\left[H_{\mathcal{A E}}, \hat{A}\right]=0
$$

immediately implies that $\hat{A}$ is a control, and its eigenstates will be preserved.

\section{Decoherence of a single qubit}

An example of continuous decoherence is afforded by a two-state apparatus $\mathcal{A}$ interacting with an environment of $N$ other spins (Zurek, 1982). The two apparatus states are $\{|\Uparrow\rangle,|\Downarrow\rangle\}$. For the simplest, yet already interesting example the self-Hamiltonian of the apparatus disappears, $H_{\mathcal{A}}=0$, and the interaction has the form:

$$
\begin{aligned}
H_{\mathcal{A E}}= & (|\Uparrow\rangle\langle\Uparrow|-| \Downarrow\rangle\langle\Downarrow|) \\
& \otimes \sum_{k} g_{k}(|\uparrow\rangle\langle\uparrow|-| \downarrow\rangle\langle\downarrow|)_{k},
\end{aligned}
$$

Under the influence of this Hamiltonian the initial state:

$$
|\Phi(0)\rangle=(a|\Uparrow\rangle+b|\Downarrow\rangle) \prod_{k=1}^{N}\left(\alpha_{k}|\uparrow\rangle_{k}+\beta_{k}|\downarrow\rangle_{k}\right)
$$

evolves into:

$$
\begin{gathered}
|\Phi(t)\rangle=a|\Uparrow\rangle\left|\mathcal{E}_{\Uparrow}(t)\right\rangle+b|\Downarrow\rangle\left|\mathcal{E}_{\Downarrow}(t)\right\rangle ; \\
\left.\left|\mathcal{E}_{\Uparrow}(t)\right\rangle=\prod_{k=1}^{N}\left(\alpha_{k} e^{i g_{k} t}|\uparrow\rangle_{k}+\beta_{k} e^{-i g_{k} t}|\downarrow\rangle_{k}\right)=\mid \mathcal{E}_{\Downarrow}(-(t) 2) t .9\right)
\end{gathered}
$$

The reduced density matrix is:

$$
\begin{aligned}
\rho_{\mathcal{A}} & =|a|^{2}|\Uparrow\rangle\left\langle\Uparrow\left|+a b^{*} r(t)\right| \Uparrow\right\rangle\langle\Downarrow| \\
& +a^{*} b r^{*}(t)|\Downarrow\rangle\left\langle\left.\Uparrow|+| b\right|^{2} \mid \Downarrow\right\rangle\langle\Downarrow| .
\end{aligned}
$$

The coefficient $r(t)=\left\langle\mathcal{E}_{\Uparrow} \mid \mathcal{E}_{\Downarrow}\right\rangle$ determines the relative size of the off-diagonal terms. It is given by:

$$
r(t)=\prod_{k=1}^{N}\left[\cos 2 g_{k} t+i\left(\left|\alpha_{k}\right|^{2}-\left|\beta_{k}\right|^{2}\right) \sin 2 g_{k} t\right] .
$$

For large environments consisting of many $(N)$ spins at large times the off-diagonal terms are typically small:

$$
|r(t)|^{2} \simeq 2^{-N} \prod_{k=1}^{N}\left[1+\left(\left|\alpha_{k}\right|^{2}-\left|\beta_{k}\right|^{2}\right)^{2}\right]
$$

The density matrix of any two-state system can be represented by a point in the $3-\mathrm{D}$ space. In terms of the coefficients $a, b$, and $r(t)$ coordinates of the point representing it are; $z=\left(|a|^{2}-|b|^{2}\right), x=\Re\left(a b^{*} r\right), y=$ $\Im\left(a b^{*} r\right)$. When the state is pure, $x^{2}+y^{2}+z^{2}=1$ : Pure states lie on the surface of the Bloch sphere (Fig. 3).

Any conceivable (unitary or non-unitary) quantum evolution can be thought of as a transformation of the surface of the pure states into the ellipsoid contained inside the Bloch sphere. Deformation of the Bloch sphere surface caused by decoherence is a special case of such general evolutions (Zurek, 1982, 1983; Berry, 1995). Decoherence does not affect $|a|$ or $|b|$. Hence, evolution due to decoherence alone occurs in plane $z=$ const. Such a "slice" through the Bloch sphere would show the point representing the state at a fraction $|r(t)|$ of its maximum distance. The complex $r(t)$ can be expressed as the sum of complex phase factors rotating with the frequencies given by differences $\Delta \omega_{j}$ between the energy eigenvalues of the interaction Hamiltonian, weighted with the probabilities of finding them in the initial state:

$$
r(t)=\sum_{j=1}^{2^{N}} p_{j} \exp \left(-i \Delta \omega_{j} t\right) .
$$

The index $j$ denotes the environment part of the energy eigenstates of the interaction Hamiltonian, Eq. (4.7): $|j\rangle=|\uparrow\rangle_{1} \otimes|\downarrow\rangle_{2} \otimes \ldots \otimes|\uparrow\rangle_{N}$. The corresponding differences between the energies of the eigenstates $|\Uparrow\rangle|j\rangle$ and $|\Downarrow\rangle|j\rangle$ are; $\Delta \omega_{j}=\left\langle\Uparrow\left|\left\langle j\left|H_{\mathcal{A E}}\right| j\right\rangle\right| \Downarrow\right\rangle$. There are $2^{N}$ distinct $|j\rangle$ 's, and, barring degeneracies, the same number of different $\Delta \omega_{j}$ 's. Probabilities $p_{j}$ are:

$$
p_{j}=|\langle j \mid \mathcal{E}(t=0)\rangle|^{2},
$$


which is in turn easily expressed in terms of the appropriate squares of $\alpha_{k}$ and $\beta_{k}$.

The evolution of $r(t)$, Eq. (4.14), is a consequence of the rotations of the complex vectors $p_{k} \exp \left(-i \Delta \omega_{j} t\right)$ with different frequencies. The resultant $r(t)$ will then start with the amplitude 1, and quickly "crumble" to

$$
\left\langle|r(t)|^{2}\right\rangle \sim \sum_{j=1}^{2^{N}} p_{j}^{2} \sim 2^{-N} .
$$

In this sense, decoherence is exponentially effective - the magnitude of the off-diagonal terms decreases exponentially fast with the physical size $N$ of the environment effectively coupled to the state of the system.

We note that the effectiveness of einselection depends on the initial state of the environment: When $\mathcal{E}$ is in the $k$ 'th eigenstate of $H_{\mathcal{A E}}, p_{j}=\delta_{j k}$, the coherence in the system will be retained. This special environment state is, however, unlikely in realistic circumstances.

\section{The classical domain and a quantum halo}

Geometry of flows induced by decoherence in the Bloch sphere exhibits characteristics encountered in general:

(i) The classical set of the einselected pointer states $(\{|\Uparrow\rangle,|\Downarrow\rangle\}$ in our case). Pointer states are the pure states least affected by decoherence.

(ii) Classical domain consisting of all the pointer states and their mixtures. In Fig. 3 it corresponds to the section $[-1,+1]$ of $z$-axis.

(iii) The quantum domain - the rest of the volume of the Bloch sphere - consisting of more general density matrices.

Visualizing decoherence-induced decomposition of the Hilbert space may be possible only in the simple case studied here, but whenever decoherence leads to classicality, emergence of generalized and often approximate version of the elements (i) - (iii) is an expected feature.

As a result of decoherence the part of the Hilbert space outside of the classical domain is "ruled out" by einselection. The severity of the prohibition on its states varies. One may measure "non-classicality" of (pure or mixed) states by quantifying their distance from this classical state with the rate of entropy production and comparing it with the much lower rate in the classical domain. Classical pointer states would be then enveloped by a "quantum halo" (Anglin and Zurek, 1996) of nearby, relatively decoherence - resistant but still somewhat quantum states, with a more flagrantly quantum (and more fragile) Schrödinger cat states further away.

By the same token, one can define einselection - induced metric in the classical domain, with the distance between two pointer states given by the rate of entropy production of their superposition. This is not the only way to define a distance: As we shall see in Section VII, redundancy of the record of a state imprinted on the environment is a very natural measure of its classicality. In course of decoherence, pointer states tend to be recorded redundantly and can be deduced by intercepting a very small fraction of the environment (Zurek, 2000; Dalvit, Dziarmaga and Zurek, 2001). One can then define distance using the fraction of the environment that needs to be intercepted to distinguish between two pointer states of the system (Ollivier, Poulin, and Zurek, 2002).

\section{Einselection and controlled shifts}

Discussion of decoherence can be generalized to the situation where the system, the apparatus, and the environment have many states, and their interactions are more complicated. Here we assume that the system is isolated, and that it interacts with the apparatus in a c-shift manner discussed in Section II. As a result of that interaction the state of the apparatus becomes entangled with the state of the system: $\left(\sum_{i} \alpha_{i}\left|s_{i}\right\rangle\right)\left|A_{0}\right\rangle \longrightarrow$ $\sum_{i} \alpha_{i}\left|s_{i}\right\rangle\left|A_{i}\right\rangle$. This state suffers from the basis ambiguity: The $\mathcal{S}-\mathcal{A}$ entanglement implies that for any state of either there exists a corresponding pure state of its partner. Indeed, when the initial state of $\mathcal{S}$ is chosen to be one of the eigenstates of the conjugate basis:

$$
\left|r_{l}\right\rangle=N^{-\frac{1}{2}} \sum_{k=0}^{N-1} \exp (2 \pi i k l / N)\left|s_{k}\right\rangle,
$$

c-shift could equally well represent a measurement of the apparatus (in the basis conjugate to $\left\{\left|A_{k}\right\rangle\right\}$ ) by the system. Thus, it is not just the basis which is ambiguous: Also the roles of the control (system) and of the target (apparatus) can be reversed when the conjugate basis is selected. These ambiguities can be removed by recognizing the role of the environment.

Figure 4 captures the essence of the idealized decoherence process, that allows the apparatus to be - in spite of the interaction with the environment - a noiseless classical communication channel (Schumacher, 1996; Lloyd, 1997). This is possible because the $\mathcal{A}-\mathcal{E}$ c-shifts do not disturb pointer states.

The advantage of this caricature of the decoherence process as a sequence of c-shifts lies in its simplicity. However, the actual process of decoherence is usually continuous (so that it can be only approximately broken up into discrete c-shifts). Moreover, in contrast to the c-nots used in quantum logic circuits, the record inscribed in the environment is usually distributed over many degrees of freedom. Last not least, the observable of the apparatus (or any other open system) may be subject to noise (and not just decoherence) or its selfHamiltonian may rotate instantaneous pointer states into their superpositions. These very likely complications will be investigated in specific models below.

Decoherence is caused by a premeasurement - like process carried out by the environment $\mathcal{E}$ :

$$
\left|\Psi_{\mathcal{S A}}\right\rangle\left|\varepsilon_{0}\right\rangle=\left(\sum_{j} \alpha_{j}\left|s_{j}\right\rangle\left|A_{j}\right\rangle\right)\left|\varepsilon_{0}\right\rangle
$$




$$
\longrightarrow \sum_{j} \alpha_{j}\left|s_{j}\right\rangle\left|A_{j}\right\rangle\left|\varepsilon_{j}\right\rangle=\left|\Phi_{\mathcal{S A E}}\right\rangle
$$

Decoherence leads to einselection when the states of the environment $\left|\varepsilon_{j}\right\rangle$ corresponding to different pointer states become orthogonal:

$$
\left\langle\varepsilon_{i} \mid \varepsilon_{j}\right\rangle=\delta_{i j}
$$

Then the Schmidt decomposition of the state vector $\left|\Phi_{\mathcal{S} \mathcal{A E}}\right\rangle$ into a composite subsystem $\mathcal{S} \mathcal{A}$ and $\mathcal{E}$ yields product states $\left|s_{j}\right\rangle\left|A_{j}\right\rangle$ as partners of the orthogonal environment states. The decohered density matrix describing $\mathcal{S A}$ pair is then diagonal in product states: For simplicity we shall often discard reference to the object that

$$
\begin{aligned}
\rho_{\mathcal{S} \mathcal{A}}^{D} & =\sum_{j}\left|\alpha_{j}\right|^{2}\left|s_{j}\right\rangle\left\langle s_{j}|| A_{j}\right\rangle\left\langle A_{j}\right| \\
& =\operatorname{Tr}_{\mathcal{E}}\left|\Phi_{\mathcal{S} \mathcal{A} \mathcal{E}}\right\rangle\left\langle\Phi_{\mathcal{S} \mathcal{A} \mathcal{E}}\right|
\end{aligned}
$$

does not interact with the environment (here - the system $\mathcal{S})$. Nevertheless, preservation of the $\mathcal{S} \mathcal{A}$ correlations is the criterion defining the pointer basis. Invoking it would get rid of many a confusion (see, e.g, discussions in Halliwell, Perez-Mercader, and Zurek, 1994; Venugopalan, 1994). The density matrix of a single object in contact with the environment will be always diagonal in an (instantaneous) Schmidt basis. This instantaneous diagonality should not be used the sole criterion for classicality (although see Zeh, 1973, 1990; Albrecht, 1992\&1993). Rather, ability of certain states to retain correlations in spite of the coupling to the environment is decisive.

When the interaction with the apparatus has the form:

$$
H_{\mathcal{A E}}=\sum_{k, l, m} g_{k l m}^{\mathcal{A E}}\left|A_{k}\right\rangle\left\langle A_{k}|| \varepsilon_{l}\right\rangle\left\langle\varepsilon_{m}\right|+\text { h.c. }
$$

the basis $\left\{\left|A_{k}\right\rangle\right\}$ is left unperturbed and any correlation with the states $\left\{\left|A_{k}\right\rangle\right\}$ is preserved. But, by definition, pointer states preserve correlations in spite of decoherence, so that any observable $A$ co-diagonal with the interaction Hamiltonian will be pointer observable. For, when the interaction is a function of $A$, it can be expanded in $A$ as a power series, so it commutes with $A$ :

$$
\left[H_{\mathcal{A E}}(A), A\right]=0
$$

The dependence of the interaction Hamiltonian on the observable is an obvious precondition for the monitoring of that observable by the environment. This admits existence of degenerate pointer eigenspaces of $A$.

\section{B. Einselection as the selective loss of information}

Establishment of the measurement-like correlation between the apparatus and the environment changes the density matrix from the premeasurement $\rho_{\mathcal{S} \mathcal{A}}^{P}$ to the decohered $\rho_{\mathcal{S} \mathcal{A}}^{D}$, Eq. (4.20). For the initially pure $\left|\Psi_{\mathcal{S A}}\right\rangle$,
Eq. (4.18), this transition is represented by:

$$
\begin{aligned}
& \rho_{\mathcal{S} \mathcal{A}}^{P}=\sum_{i, j} \alpha_{i} \alpha_{j}^{*}\left|s_{i}\right\rangle\left\langle s_{j}|| A_{i}\right\rangle\left\langle A_{j}\right| \longrightarrow \\
& \longrightarrow \sum_{i}\left|\alpha_{i}\right|^{2}\left|s_{i}\right\rangle\left\langle s_{i}|| A_{i}\right\rangle\left\langle A_{i}\right|=\rho_{\mathcal{S} \mathcal{A}}^{D}
\end{aligned}
$$

Einselection is accompanied by the increase of entropy:

$$
\Delta H\left(\rho_{\mathcal{S A}}\right)=H\left(\rho_{\mathcal{S} \mathcal{A}}^{D}\right)-H\left(\rho_{\mathcal{S} \mathcal{A}}^{P}\right) \geq 0
$$

and by the disappearance of the ambiguity in what was measured (Zurek, 1981, 1993a). Thus, before decoherence the conditional density matrices of the system $\left.\rho_{\mathcal{S} \mid C_{j}}\right\rangle$ are pure for any state $\left|C_{j}\right\rangle$ of the apparatus pointer. They are defined using the unnormalized:

$$
\tilde{\rho}_{\mathcal{S} \mid \Pi_{j}}=\operatorname{Tr}_{\mathcal{A}} \Pi_{j} \rho_{\mathcal{S} \mathcal{A}}
$$

where in the simplest case $\Pi_{j}=\left|C_{j}\right\rangle\left\langle C_{j}\right|$ projects onto a pure state of the apparatus. ${ }^{4}$

Normalized $\rho_{\mathcal{S} \mid \Pi_{j}}$ can be obtained by using the probability of the outcome:

$$
\rho_{\mathcal{S} \mid \Pi_{j}}=p_{j}^{-1} \tilde{\rho}_{\mathcal{S} \mid \Pi_{j}} ; \quad p_{j}=\operatorname{Tr} \tilde{\rho}_{\mathcal{S} \mid \Pi_{j}} .
$$

Conditional density matrix represents the description of the system $\mathcal{S}$ available to the observer who knows that the apparatus $\mathcal{A}$ is in a subspace defined by $\Pi_{j}$.

Before decoherence, $\rho_{\mathcal{S}\left|C_{j}\right\rangle}^{P}$ is pure for any state $\left|C_{j}\right\rangle$ :

$$
\left(\rho_{\mathcal{S} \mid \Pi_{j}}^{P}\right)^{2}=\rho_{\mathcal{S} \mid \Pi_{j}}^{P} \quad \forall\left|C_{j}\right\rangle
$$

providing the initial premeasurement state, Eq. (4.23), was pure as well. It follows that:

$$
H\left(\rho_{\mathcal{S} \mathcal{A}\left|C_{j}\right\rangle}^{P}\right)=0 \quad \forall\left|C_{j}\right\rangle .
$$

For this same case given by the initially pure $\rho_{\mathcal{S} \mathcal{A}}^{P}$ of Eq. (4.23), conditional density matrices obtained from the decohered $\rho_{\mathcal{S} \mathcal{A}}^{D}$ will be pure if and only if they are conditioned upon the pointer states $\left\{\left|A_{k}\right\rangle\right\}$;

$$
\begin{gathered}
\left(\rho_{\mathcal{S}\left|C_{j}\right\rangle}^{D}\right)^{2}=\rho_{\mathcal{S}\left|C_{j}\right\rangle}^{P}=\left|s_{k}\right\rangle\left\langle s_{k}|\Longleftrightarrow| C_{j}\right\rangle=\left|A_{j}\right\rangle ; \\
H\left(\rho_{\mathcal{S}\left|A_{j}\right\rangle}^{D}\right)=H\left(\rho_{\mathcal{S}\left|A_{j}\right\rangle}^{P}\right)
\end{gathered}
$$

\footnotetext{
4 This can be generalized to projections onto multidimensional subspaces of the apparatus. In that case, purity of the conditional density matrix will be usually lost during the trace over the states of the pointer. This is not surprising: When the observer reads off the pointer of the apparatus only in a coarsegrained manner, he will forgo part of the information about the system. Amplification we have considered before can prevent some of such loss of resolution due to coarse graining in the apparatus. Generalizations to density matrices conditioned upon projection operator valued measures (POVM's) (Kraus, 1983) are also possible.
} 
This last equation is valid even when the initial states of the system and of the apparatus are not pure. Thus, only in the pointer basis the pre-decoherence strength of the $\mathcal{S} \mathcal{A}$ correlation will be maintained. In all other bases:

$$
\begin{aligned}
& \operatorname{Tr}\left(\rho_{\mathcal{S}\left|C_{j}\right\rangle}^{D}\right)^{2}<\operatorname{Tr} \rho_{\mathcal{S}\left|C_{j}\right\rangle}^{D} ; \quad\left|C_{j}\right\rangle \notin\left\{\left|A_{j}\right\rangle\right\} \\
& H\left(\rho_{\mathcal{S}\left|C_{j}\right\rangle}^{P}\right)<H\left(\rho_{\mathcal{S}\left|C_{j}\right\rangle}^{D}\right) ; \quad\left|C_{j}\right\rangle \notin\left\{\left|A_{j}\right\rangle\right\}
\end{aligned}
$$

In particular, in the basis $\left\{\left|B_{j}\right\rangle\right\}$ conjugate to the pointer states $\left\{\left|A_{j}\right\rangle\right\}$, Eq. (2.14), there is no correlation left with the state of the system, that is:

$$
\rho_{\mathcal{S}\left|B_{j}\right\rangle}^{D}=N^{-1} \sum_{k}\left|s_{k}\right\rangle\left\langle s_{k}\right|=\mathbf{1} / N
$$

where $\mathbf{1}$ is a unit density matrix. Consequently;

$$
\begin{gathered}
\left(\rho_{\mathcal{S}\left|B_{j}\right\rangle}^{D}\right)^{2}=\rho_{\mathcal{S}\left|B_{j}\right\rangle} / N \\
H\left(\rho_{\mathcal{S}\left|B_{j}\right\rangle}^{D}\right)=H\left(\rho_{\mathcal{S}\left|B_{j}\right\rangle}^{P}\right)-\lg N=-\lg N .
\end{gathered}
$$

Note that, initially, conditional density matrices were pure also in the conjugate (and any other) basis, provided that the initial state was the pure entangled projection operator $\rho_{\mathcal{S} \mathcal{A}}^{P}=\left|\Psi_{\mathcal{S A}}\right\rangle\left\langle\Psi_{\mathcal{S A}}\right|$, Eq. (4.23).

\section{Mutual information and discord}

Selective loss of information everywhere except in the pointer states is the essence of einselection. It is reflected in the change of the mutual information which starts from:

$$
\begin{aligned}
\mathcal{I}^{P}(\mathcal{S}: \mathcal{A}) & =H\left(\rho_{\mathcal{S}}^{P}\right)+H\left(\rho_{\mathcal{A}}^{P}\right)-H\left(\rho_{\mathcal{S}, \mathcal{A}}^{P}\right) \\
& =-2 \sum_{i}\left|\alpha_{i}\right|^{2} \lg \left|\alpha_{i}\right|^{2}
\end{aligned}
$$

As a result of einselection, for initially pure cases, it decreases to at most half its initial value:

$$
\begin{aligned}
\mathcal{I}^{D}(\mathcal{S}: \mathcal{A}) & =H\left(\rho_{\mathcal{S}}^{D}\right)+H\left(\rho_{\mathcal{A}}^{D}\right)-H\left(\rho_{\mathcal{S}, \mathcal{A}}^{D}\right) \\
& =-\sum_{i}\left|\alpha_{i}\right|^{2} \lg \left|\alpha_{i}\right|^{2}
\end{aligned}
$$

This level is reached when the pointer basis coincides with the Schmidt basis of $\left|\Psi_{\mathcal{S A}}\right\rangle$. The decrease in the mutual information is due to the increase of the joint

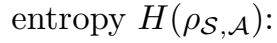

$$
\begin{aligned}
\Delta \mathcal{I}(\mathcal{S}: \mathcal{A}) & =\mathcal{I}^{P}(\mathcal{S}: \mathcal{A})-\mathcal{I}^{D}(\mathcal{S}: \mathcal{A}) \\
& =H\left(\rho_{\mathcal{S}, \mathcal{A}}^{D}\right)-H\left(\rho_{\mathcal{S}, \mathcal{A}}^{P}\right)=\Delta H\left(\rho_{\mathcal{S}, \mathcal{A}}\right)
\end{aligned}
$$

Classically, equivalent definition of the mutual information obtains from the asymmetric formula:

$$
\mathcal{J}_{\mathcal{A}}(\mathcal{S}: \mathcal{A})=H\left(\rho_{\mathcal{S}}\right)-H\left(\rho_{\mathcal{S} \mid \mathcal{A}}\right)
$$

with the help of the conditional entropy $H\left(\rho_{\mathcal{S} \mid \mathcal{A}}\right)$. Above, subscript $\mathcal{A}$ indicates the member of the correlated pair that will be the source of the information about its partner. A symmetric counterpart of the above equation, $\mathcal{J}_{\mathcal{S}}(\mathcal{S}: \mathcal{A})=H\left(\rho_{\mathcal{A}}\right)-H\left(\rho_{\mathcal{A} \mid \mathcal{S}}\right)$, can be also written.

In the quantum case, definition of Eq. (4.32) is so far incomplete, as a quantum analogue of the classical conditional information has not been yet specified. Indeed, Eqs. (4.30a) and (4.32) jointly imply that in the case of entanglement a quantum conditional entropy $H\left(\rho_{\mathcal{S} \mid \mathcal{A}}\right)$ would have to be negative! For, in this case;

$$
H\left(\rho_{\mathcal{S} \mid \mathcal{A}}\right)=\sum_{i}\left|\alpha_{i}\right|^{2} \lg \left|\alpha_{i}\right|^{2}<0
$$

would be required to allow for $\mathcal{I}(\mathcal{S}: \mathcal{A})=\mathcal{J}_{\mathcal{A}}(\mathcal{S}: \mathcal{A})$. Various quantum redefinitions of $\mathcal{I}(\mathcal{S}: \mathcal{A})$ or $H\left(\rho_{\mathcal{S} \mid \mathcal{A}}\right)$ have been proposed to address this (Lieb, 1975; Schumacher and Nielsen, 1996; Lloyd, 1997; Cerf and Adami, 1997). We shall simply regard this fact as an illustration of the strength of quantum correlations (i. e., entanglement), which allow $\mathcal{I}(\mathcal{S}: \mathcal{A})$ to violate the inequality:

$$
\mathcal{I}(\mathcal{S}: \mathcal{A}) \leq \min \left(H_{\mathcal{S}}, H_{\mathcal{A}}\right)
$$

This inequality follows directly from Eq. (4.32) and the non-negativity of classical conditional entropy (see e.g. Cover and Thomas, 1991).

Decoherence decreases $\mathcal{I}(\mathcal{S}: \mathcal{A})$ to this allowed level (Zurek, 1983). Moreover, now the conditional entropy can be defined in the classical pointer basis as the average of partial entropies computed from the conditional $\rho_{\mathcal{S}\left|A_{i}\right\rangle}^{D}$ over the probabilities of different outcomes:

$$
H\left(\rho_{\mathcal{S} \mid \mathcal{A}}\right)=\sum_{i} p_{\left|A_{i}\right\rangle} H\left(\rho_{\mathcal{S}\left|A_{i}\right\rangle}^{D}\right)
$$

Prior to decoherence, the use of probabilities would not have been legal.

For the case considered here, Eq. (4.18), the conditional entropy $H\left(\rho_{\mathcal{S} \mid \mathcal{A}}\right)=0$ : In the pointer basis there is a perfect correlation between the system and the apparatus, providing that the premeasurement Schmidt basis and the pointer basis coincide. Indeed, it is tempting to define a good apparatus or a classical correlation by insisting on such a coincidence.

The difficulties with conditional entropy and mutual information are highly symptomatic. The trouble with $H\left(\rho_{\mathcal{S} \mid \mathcal{A}}\right)$ arises for states that exhibit quantum correlations - entanglement of $\left|\Psi_{\mathcal{S} \mathcal{A}}\right\rangle$ being an extreme example - and, thus, do not admit an interpretation based on probabilities. A useful sufficient condition for the classicality of correlations is then the existence of an apparatus basis that allows quantum versions of the two classically identical expressions for the mutual information to coincide, $\mathcal{I}(\mathcal{S}: \mathcal{A})=\mathcal{J}_{\mathcal{A}}(\mathcal{S}: \mathcal{A})$, (Zurek, 2000b; 2002a; Ollivier and Zurek, 2002): Equivalently, the discord

$$
\delta \mathcal{I}_{\mathcal{A}}(\mathcal{S} \mid \mathcal{A})=\mathcal{I}(\mathcal{S}: \mathcal{A})-\mathcal{J}_{\mathcal{A}}(\mathcal{S}: \mathcal{A})
$$


must vanish: Unless $\delta \mathcal{I}_{\mathcal{A}}(\mathcal{S} \mid \mathcal{A})=0$, probabilities of the distinct apparatus pointer states cannot exist.

We end this subsection with a part summary, part anticipatory remarks: Pointer states retain undiminished correlations with the measured system $\mathcal{S}$, or with any other system, including observers. The loss of information caused by decoherence is given by Eq. (4.31). It was precisely such as to lift conditional information from the paradoxical (negative) values to the classically allowed level, Eq. (4.33). This is equal to the information gained by the observer when he consults the apparatus pointer. This is no coincidence - the environment has 'measured' (became correlated with) the apparatus in the very same pointer basis in which observers have to access $\mathcal{A}$ to take advantage of the remaining (classical) correlation between the pointer and the system. Only when observers and the environment monitor co-diagonal observables they do not get in each others way.

In the idealized case, preferred basis was distinguished by its ability to retain perfect correlations with the system in spite of decoherence. This remark shall serve as a guide in other situations: It will lead to a criterion - predictability sieve - used to identify preferred states in less idealized circumstances. For example, when the self-Hamiltonian of the system is non-trivial, or when the commutation relation, Eq. (4.22), does not hold exactly for any observable, we shall seek states that are best in retaining correlations with the other systems.

\section{Decoherence, entanglement, dephasing, and noise}

In the symbolic representation of Fig. 4, noise is the process in which environment acts as a control, inscribing information about its state in the state of the system, that assumes the role of the target. However, the direction of the information flow in c-nots and c-shifts depends on the choice of initial states. Control and target switch roles when, for a given Hamiltonian of interaction, one prepares input of the c-not in the basis conjugate to the logical "pointer" states. Einselected states correspond to the set which - when used in c-nots or c-shifts - minimizes the effect of interactions directed from the environment to the system.

Einselection is caused by the premeasurement carried out by the environment on the pointer states. Decoherence follows from the Heisenberg's indeterminacy: Pointer observable is measured by the environment. Therefore, the complementary observable must become at least as indeterminate as is demanded by the Heisenberg's principle. As the environment and the systems entangle through an interaction that favors a set of pointer states, their phases become indeterminate (see Eq. (4.29) and discussion of envariance in section VI). Decoherence can be thought of as the resulting loss of phase relations.

Observers can be ignorant of phases for reasons that do not lead to an imprint of the state of the system on the environment. Classical noise can cause such dephas- ing when the observer does not know the time-dependent classical perturbation Hamiltonian responsible for this unitary, but unknown evolution. For example, in the pre-decoherence state vector, Eq. (4.18), random phase noise will cause a transition:

$$
\begin{aligned}
& \left|\Psi_{\mathcal{S A}}\right\rangle=\left(\sum_{j} \alpha_{j}\left|s_{j}\right\rangle\left|A_{j}\right\rangle\right) \\
& \longrightarrow \sum_{j} \alpha_{j} \exp \left(i \phi_{j}^{(n)}\right)\left|s_{j}\right\rangle\left|A_{j}\right\rangle=\left|\Psi_{\mathcal{S} \mathcal{A}}^{(n)}\right\rangle .
\end{aligned}
$$

A dephasing Hamiltonian acting either on the system or on the apparatus can lead to such an effect. In this second case its form could be:

$$
H_{D}^{(n)}=\sum_{j} \dot{\phi}_{j}^{(n)}(t)\left|A_{j}\right\rangle\left\langle A_{j}\right| .
$$

In contrast to interactions causing premeasurements, entanglement, and decoherence, $H_{D}$ cannot influence the nature or the degree of the $\mathcal{S A}$ correlations: $H_{D}$ does not imprint the state of $\mathcal{S}$ or $\mathcal{A}$ anywhere else in the Universe: For each individual realization $n$ of the phase noise (each selection of $\left\{\phi_{j}^{(n)}(t)\right\}$ in Eq. (4.37)) the state $\left|\Phi_{\mathcal{S A}}^{(n)}\right\rangle$ remains pure. Given only $\left\{\phi_{j}^{(n)}\right\}$ one could restore predephasing state on a case - by - case basis. However, in absence of such detailed information, one is often forced to represent $\mathcal{S} \mathcal{A}$ by the density matrix averaged over the ensemble of noise realizations:

$$
\begin{aligned}
& \bar{\rho}_{\mathcal{S} \mathcal{A}}=\left\langle\mid \Psi_{\mathcal{S} \mathcal{A}}\right\rangle\left\langle\Psi_{\mathcal{S A}} \mid\right\rangle=\sum_{j}\left|\alpha_{j}\right|^{2}\left|s_{j}\right\rangle\left\langle s_{j}|| A_{j}\right\rangle\left\langle A_{j}\right| \\
& +\sum_{j, k} \sum_{n} e^{i\left(\phi_{j}^{(n)}-\phi_{k}^{(n)}\right)} \alpha_{j} \alpha_{k}\left|s_{j}\right\rangle\left\langle s_{k}|| A_{j}\right\rangle\left\langle A_{k}\right|
\end{aligned}
$$

In this phase - averaged density matrix off-diagonal terms may disappear. Nevertheless, each member of the ensemble may exist in a state as pure as it was before dephasing. NMR offers examples of dephasing (which can be reversed using spin echo). Dephasing is a loss of phase coherence due to noise in phases. It does not result in an information transfer to the environment.

Dephasing cannot be used to justify existence of preferred basis in individual quantum systems. Nevertheless, the ensemble as a whole may obey the same master equation as individual systems entangling with the environment. Indeed, many of the symptoms of decoherence arise in this setting. Thus, in spite of the light shed on this issue by the discussion of simple cases (Wootters and Zurek, 1979; Stern, Aharonov, and Imry, 1989) more remains to be understood, perhaps by considering implications of envariance (see Section VI).

Noise is an even more familiar and less subtle effect represented by transitions that break one-to-one correspondence in Eq. (4.39). Noise in the apparatus would cause a random rotation of states $\left|A_{j}\right\rangle$. It could be modelled by a collection of Hamiltonians similar to $H_{D}^{(n)}$ but not co-diagonal with the observable of interest. Then, after an ensemble average similar to Eq. (4.39), one-to-one 
correspondence between $\mathcal{S}$ and $\mathcal{A}$ would be lost. However - as before - the evolution is unitary for each $n$, and the unperturbed state could be reconstructed from the information observer could have in advance.

Hence, in case of dephasing or noise information about their cause obtained either in advance, or afterwards, suffices to undo their effect. Decoherence relies on entangling interactions (although, strictly speaking, it need not invlove entanglement (Eisert and Plenio, 2002). Thus, neither prior nor posterior knowledge of the state of environment is enough. Transfer of information about the decohering system to the environment is essential, and plays key role in the interpretation.

We note that, while nomenclature used here seems most sensible to this author and is widely used, it is unfortunately not universal. For example, in the context of quantum computation "decoherence" is sometimes used to describe any process that can cause errors (but see related discussion in Nielsen and Chuang, 2000).

\section{Predictability sieve and einselection}

Evolution of a quantum system prepared in a classical state should emulate classical evolution that can be idealized as a "trajectory" - a predictable sequence of objectively existing states. For a purely unitary evolution, all of the states in the Hilbert space retain their purity and are therefore equally predictable. However, in the presence of an interaction with the environment, a generic superposition representing correlated states of the system and of the apparatus will decay into a mixture diagonal in pointer states, Eq. (4.23). Only when the pre-decoherence state of $\mathcal{S} \mathcal{A}$ is a product of a single apparatus pointer state $\left|A_{i}\right\rangle$ with the corresponding outcome state of the system (or a mixture of such product states), decoherence has no effect:

$$
\rho_{\mathcal{S} \mathcal{A}}^{P}=\left|s_{i}\right\rangle\left\langle s_{i}|| A_{i}\right\rangle\left\langle A_{i}\right|=\rho_{\mathcal{S} \mathcal{A}}^{D}
$$

A correlation of a pointer state with any state of an isolated system is untouched by the environment. By the same token, when the observer prepares $\mathcal{A}$ in the pointer state $\left|A_{i}\right\rangle$, he can count on it remaining pure. One can even think of $\left|s_{i}\right\rangle$ as the record of the pointer state of $\mathcal{A}$. Einselected states are predictable: they preserve correlations, and hence are effectively classical.

In the above idealized cases predictability of some states follows directly from the structure of the relevant Hamiltonians (Zurek, 1981). Correlation with a subspace associated with a projection operator $P_{A}$ will be immune to decoherence providing that:

$$
\left[H_{\mathcal{A}}+H_{\mathcal{A E}}, P_{A}\right]=0
$$

In more realistic cases it is difficult to demand exact conservation guaranteed by such a commutation condition. Looking for approximate conservation may still be a good strategy. Various densities used in hydrodynamics are one obvious choice (Gell-Mann and Hartle, 1990, 1994).
In general, it is useful to invoke a more fundamental predictability criterion (Zurek, 1993a). One can measure the loss of predictability caused by the evolution for every pure state $|\Psi\rangle$ by von Neumann entropy or some other measure of predictability such as the purity:

$$
\varsigma_{\Psi}(t)=\operatorname{Tr} \rho_{\Psi}^{2}(t)
$$

In either case, predictability is a function of time and a functional of the initial state as $\rho_{\Psi}(0)=|\Psi\rangle\langle\Psi|$. Pointer states are obtained by maximizing predictability functional over $|\Psi\rangle$. When decoherence leads to classicality, good pointer states exist, and the answer is robust.

Predictability sieve sifts all of the Hilbert space, ordering states according to their predictability. The top of the list will be the most classical. This point of view allows for unification of the simple definition of the pointer states in terms of the commutation relation Eq. (4.41), with the more general criteria required to discuss classicality in other situations. The eigenstates of the exact pointer observable are selected by the sieve: Eq. (4.41) guarantees that they will retain their purity in spite of the environment, and are (somewhat trivially) predictable.

Predictability sieve can be generalized to situations where the initial states are mixed (Paraonanu, 2002). Often whole subspaces emerge from the predictability sieve, naturally leading to "decoherence-free subspaces" (see e. g. Lidar et al., 1999) and can be adapted to yield "noiseless subsystems" (which are a non-Abelian generalization of pointer states; see e.g. Zanardi, 2000; Knill, Laflamme, and Viola, 2000). However, calculations are in general quite difficult even for the initial pure state cases.

The idea of the "sieve" selecting preferred to-beclassical states is novel and only partly explored. We shall see it "in action" below. We have outlined two criteria for sifting through the Hilbert space in search for classicality: von Neumann entropy and purity define, after all, two distinct functionals. Entropy is arguably an obvious information-theoretic measure of predictability loss. Purity is much easier to compute and is often used as a "cheap substitute", and has a physical significance of its own. It seems unlikely that pointer states selected by the predictability and purity sieves could substantially differ. After all, $-\operatorname{Tr} \rho \ln \rho=\operatorname{Tr} \rho\left\{(\mathbf{1}-\rho)-(\mathbf{1}-\rho)^{2} / 2+\ldots\right.$ so that one can expect the most predictable states to also remain purest (Zurek, 1993a). However, the expansion, Eq. (4.43), is very slowly convergent. Therefore, a more mathematically satisfying treatment of the differences between the states selected by these two criteria would be desirable, especially in cases where (as we shall see in the next section for the harmonic oscillator) preferred states are coherent, and hence the classical domain forms relatively broad "mesa" in the Hilbert space.

The possible discrepancy between the states selected by the sieves based on the predictability and on the purity raises a more general question: Will all the sensible criteria yield identical answer? After all, one can imagine other reasonable criteria for classicality, such as the yet-to-be-explored "distinguishability sieve" of Schu- 
macher (1999) which picks out states whose descendants are most distinguishable in spite of decoherence. Moreover, as we shall see in Section VII (also, Zurek 2000) one can ascribe classicality to states that are most redundantly recorded by the environment. The menu of various classicality criteria already contains several positions, and more may be added in the future. There is no a priori reason to expect that all of these criteria will lead to identical sets of preferred states. It is nevertheless reasonable to hope that, in the macroscopic limit in which classicality is indeed expected, differences between various sieves should be negligible. The same stability in the selection of the classical domain is expected with respect to the changes of, say, the time of the evolution from the initial pure state: Reasonable changes of such details within the time interval where einselection is expected to be effective should lead to more or less similar preferred states, and certainly to preferred states contained within each others "quantum halo" (Anglin and Zurek, 1996). As noted above, this seems to be the case in the examples explored to date. It remains to be seen whether all criteria will agree in other situations of interest.

\section{EINSELECTION IN PHASE SPACE}

Einselection in phase space is a special, yet very important topic. It should lead to phase space points, trajectories, and to classical (Newtonian) dynamics. Special role of position in classical physics can be traced to the nature of interactions (Zurek, 1981; 1982; 1991) that depend on distance, and, therefore, commute with position (see Eq. (4.22)). Evolution of open systems includes, however, the flow in phase space induced by the self-Hamiltonian. Consequently, the set of preferred states turns out to be a compromise, localized in both position and momentum - localized in phase space.

Einselection is responsible for the classical structure of phase space. States selected by the predictability sieve become phase space "points", and their time-ordered sequences are "trajectories". In the underdamped, classically regular systems one can recover this phase space structure along with the (almost) reversible evolution. In chaotic systems there is a price to be paid for classicality: Combination of decoherence with the exponential divergence of classical trajectories (which is the defining feature of chaos) leads to entropy production at a rate given - in the classical limit - by the sum of positive Lyapunov exponents. Thus, the dynamical second law can emerge from the interplay of classical dynamics and quantum decoherence, with the entropy production caused by the information "leaking" into the environment (Zurek and Paz 1994 \& 1995a; Zurek, 1998b; Paz and Zurek 2001).

\section{A. Quantum Brownian motion}

Quantum Brownian motion model consists of an environment $\mathcal{E}$ - a collection of harmonic oscillators (coordinates $q_{n}$, masses $m_{n}$, frequencies $\omega_{n}$, and coupling constants $c_{n}$ ) interacting with the system $\mathcal{S}$ (coordinate $x)$, with a mass $M$ and a potential $V(x)$. We shall often consider harmonic $V(x)=M \Omega_{0}^{2} x^{2} / 2$, so that the whole $\mathcal{S E}$ is linear and one can obtain an exact solution. This assumption will be relaxed later.

The Lagrangian of the system-environment entity is:

$$
L\left(x, q_{n}\right)=L_{\mathcal{S}}(x)+L_{\mathcal{S E}}\left(x,\left\{q_{n}\right\}\right)
$$

The system alone has the Lagrangian:

$$
L_{\mathcal{S}}(x)=\frac{M}{2} \dot{x}^{2}-V(x)=\frac{M}{2}\left(\dot{x}^{2}-\Omega_{0}^{2} x^{2}\right) .
$$

The effect of the environment is modelled by the sum of the Lagrangians of individual oscillators and of the system-environment interaction terms:

$$
L_{\mathcal{S E}}=\sum_{n} \frac{m_{n}}{2}\left(\dot{q}_{n}^{2}-\omega_{n}^{2}\left(q_{n}-\frac{c_{n} x}{m_{n} \omega_{n}^{2}}\right)^{2}\right) .
$$

This Lagrangian takes into account renormalization of potential energy of the Brownian particle. The interaction depends (linearly) on the position $x$ of the harmonic oscillator. Hence, we expect $x$ to be an instantaneous pointer observable. In combination with the harmonic evolution this leads Gaussian pointer states - welllocalized in both $x$ and $p$. An important characteristic of the model is the spectral density of the environment:

$$
C(\omega)=\sum_{n} \frac{c_{n}^{2}}{2 m_{n} \omega_{n}} \delta\left(\omega-\omega_{n}\right)
$$

The effect of the environment can be expressed through the propagator acting on the reduced $\rho_{\mathcal{S}}$ :

$$
\rho_{\mathcal{S}}\left(x, x^{\prime}, t\right)=\int d x_{0} d x_{0}^{\prime} J\left(x, x^{\prime}, t \mid x_{0}, x_{0}^{\prime}, t_{0}\right) \rho_{\mathcal{S}}\left(x_{0}, x_{0}^{\prime}, t_{0}\right) \text {. }
$$

We focus on the case when the system and the environment are initially statistically independent, so that their density matrices in a product state:

$$
\rho_{\mathcal{S E}}=\rho_{\mathcal{S}} \rho_{\mathcal{E}}
$$

This is a restrictive assumption. One can try to justify it as an idealization of a measurement that correlates $\mathcal{S}$ with the observer and destroys correlations of $\mathcal{S}$ with $\mathcal{E}$, but that is only an approximation, as realistic measurements leave partial correlations with the environment intact. Fortunately, pre-existing post-measurement correlations lead only to minor differences in the salient features of the subsequent evolution of the system (Romero and Paz, 1997; Anglin, Paz and Zurek, 1997). 
Evolution of the whole $\rho_{\mathcal{S E}}$ can be represented as:

$$
\begin{gathered}
\rho_{\mathcal{S E}}\left(x, q, x^{\prime}, q^{\prime}, t\right)=\int d x_{0} d x_{0}^{\prime} d q_{0} d q_{0}^{\prime} \rho_{\mathcal{S E}}\left(x_{0}, q_{0}, x_{0}^{\prime}, q_{0}^{\prime}, t_{0}\right) \\
K\left(x, q, t, x_{0}, q_{0}\right) K^{*}\left(x^{\prime}, q^{\prime}, t, x_{0}^{\prime}, q_{0}^{\prime}\right)
\end{gathered}
$$

Above, we suppress the sum over the indices of the individual environment oscillators. The evolution operator $K\left(x, q, t, x_{0}, q_{0}\right)$ can be expressed as a path integral:

$$
K\left(x, q, t, x_{0}, q_{0}\right)=\int D x D q \exp \left(\frac{i}{\hbar} I[x, q]\right)
$$

where $I[x, q]$ is the action functional that depends on the trajectories $x, q$. The integration must satisfy boundary conditions:

$$
x(0)=x_{0} ; x(t)=x ; q(0)=q_{0} ; q(t)=q .
$$

The expression for the propagator of the density matrix can be now written in terms of actions corresponding to the two Lagrangians, Eqs. (5.1-3):

$$
\begin{gathered}
J\left(x, x^{\prime}, t \mid x_{0}, x_{0}^{\prime}, t_{0}\right)=\int D x D x^{\prime} \exp \frac{i}{\hbar}\left(I_{\mathcal{S}}[x]-I_{\mathcal{S}}\left[x^{\prime}\right]\right) \\
\quad \times \int d q d q_{0} d q_{0}^{\prime} \rho_{\mathcal{E}}\left(q_{0}, q_{0}^{\prime}\right) \\
\int D q D q^{\prime} \exp \frac{i}{\hbar}\left(I_{\mathcal{S E}}[x, q]-I_{\mathcal{S E}}\left[x^{\prime}, q^{\prime}\right]\right) .
\end{gathered}
$$

The separability of the initial conditions, Eq. (5.6), was used to make propagator depend only on the initial conditions of the environment. Collecting all terms containing integrals over $\mathcal{E}$ in the above expression leads to the influence functional (Feynman and Vernon, 1963):

$$
\begin{aligned}
F\left(x, x^{\prime}\right)= & \int d q d q_{0} d q_{0}^{\prime} \rho_{\mathcal{E}}\left(q_{0}, q_{0}^{\prime}\right) \\
& \int D q D q^{\prime} \exp \frac{i}{\hbar}\left(I_{\mathcal{S E}}[x, q]-I_{\mathcal{S E}}\left[x^{\prime}, q^{\prime}\right]\right)
\end{aligned}
$$

It can be evaluated explicitly for specific models of the initial density matrix of the environment.

Environment in thermal equilibrium provides a useful and tractable model for the initial state. The density matrix of the $n$ 'th mode of the thermal environment is:

$$
\begin{aligned}
& \rho_{\mathcal{E}_{n}}\left(q, q^{\prime}\right)=\frac{m_{n} \omega_{n}}{2 \pi \hbar \sinh \left(\frac{\hbar \omega_{n}}{k_{B} T}\right)} \times \exp -\left\{\frac{m_{n} \omega_{n}}{2 \pi \hbar \sinh \left(\frac{\hbar \omega_{n}}{k_{B} T}\right)}\right. \\
& \left.\times\left[\left(q_{n}^{2}+q_{n}^{\prime 2}\right) \cosh \left(\frac{\hbar \omega_{n}}{k_{B} T}\right)-2 q_{n} q_{n}^{\prime}\right]\right\} .
\end{aligned}
$$

The influence functional can be written as (Grabert, Schramm, and Ingold, 1988):

$$
\begin{aligned}
& i \ln F\left(x, x^{\prime}\right)=\int_{0}^{t} d s\left(x-x^{\prime}\right)(s) \int_{0}^{s} d u \\
& \left(\eta\left(s-s^{\prime}\right)\left(x+x^{\prime}\right)\left(s^{\prime}\right)-i \nu\left(s-s^{\prime}\right)\left(x-x^{\prime}\right)\left(s^{\prime}\right)\right)
\end{aligned}
$$

where $\nu(s)$ and $\eta(s)$ are dissipation and noise kernels, respectively, defined in terms of the spectral density:

$$
\nu(s)=\int_{0}^{\infty} d \omega C(\omega) \operatorname{coth}(\hbar \omega \beta / 2) \cos (\omega s) ;
$$

$$
\eta(s)=\int_{0}^{\infty} d \omega C(\omega) \sin (\omega s) .
$$

With the assumption of thermal equilibrium at $k_{B} T=$ $1 / \beta$, and in the harmonic oscillator case $V(x)=$ $M \Omega_{0}^{2} x^{2} / 2$, the integrand of Eq. (5.10) for the propagator is Gaussian: The integral can be computed exactly, and should also have a Gaussian form. The result can be conveniently written in terms of the diagonal and offdiagonal coordinates of the density matrix in the position representation, $X=x+x^{\prime}, Y=x-x^{\prime}$ :

$$
\begin{gathered}
J\left(X, Y, t \mid X_{0}, Y_{0}, t_{0}\right)= \\
\frac{b_{3}}{2 \pi} \frac{\exp i\left(b_{1} X Y+b_{2} X_{0} Y-b_{3} X Y_{0}-b_{4} X_{0} Y_{0}\right)}{\exp \left(a_{11} Y^{2}+2 a_{12} Y Y_{0}+a_{22} Y_{0}^{2}\right)}
\end{gathered}
$$

The time-dependent coefficients $b_{k}$ and $a_{i j}$ are computed from the noise and dissipation kernels, that reflect the properties of the environment. They obtain from the solutions of the equation:

$$
\ddot{u}(s)+\Omega_{0}^{2} u(s)+2 \int_{0}^{s} d s \eta\left(s-s^{\prime}\right) u\left(s^{\prime}\right)=0 .
$$

Two such solutions that satisfy boundary conditions $u_{1}(0)=u_{2}(t)=1$ and $u_{1}(t)=u_{2}(0)=0$ can be used for this purpose. They yield the coefficients of the Gaussian propagator through;

$$
\begin{aligned}
& b_{1(2)}(t)=\dot{u}_{2(1)}(t) / 2, \quad b_{3(4)}(t)=\dot{u}_{2(1)}(0) / 2 \\
& a_{i j}(t)=\frac{1}{1+\delta_{i j}} \int_{0}^{t} d s \int_{0}^{t} d s^{\prime} u_{i}(s) u_{j}\left(s^{\prime}\right) \nu\left(s-s^{\prime}\right)
\end{aligned}
$$

The master equation can be now obtained by taking the time derivative of the Eq. (5.5), which in effect reduces to the computation of the derivative of the propagator, Eq. (5.16) above:

$$
\begin{aligned}
\dot{J}= & \left\{\dot{b}_{3} / b_{3}+i \dot{b}_{1} X Y+i \dot{b}_{2} X_{0} Y-i \dot{b}_{3} X Y_{0}\right. \\
& \left.-i \dot{b}_{4} X_{0} Y_{0}-\dot{a}_{11} Y^{2}-\dot{a}_{12} Y Y_{0}-\dot{a}_{22} Y_{0}^{2}\right\} J
\end{aligned}
$$

The time derivative of $\rho_{\mathcal{S}}$ can be obtained by multiplying the operator on the right hand side by an initial density matrix and integrating over the initial coordinates $X_{0}, Y_{0}$. Given the form of Eq. (5.19), one may expect that this procedure will yield an integro-differential (non-local in time) evolution operator for $\rho_{\mathcal{S}}$. However, time dependence of the evolution operator disappears as a result of two identities satisfied by the propagator:

$$
Y_{0} J=\left(\frac{b_{1}}{b_{3}} Y+\frac{i}{b_{3}} \partial_{X}\right) J,
$$




$$
\begin{aligned}
X_{0} J= & \left(-\frac{b_{1}}{b_{2}} X-\frac{i}{b_{2}} \partial_{Y}\right. \\
& \left.-i\left(\frac{2 a_{11}}{b_{2}}+\frac{a_{12} b_{1}}{b_{2} b_{3}}\right) Y+\frac{a_{12}}{b_{2} b_{3}} \partial_{X}\right) J .
\end{aligned}
$$

After the appropriate substitutions, the resulting equation with renormalized Hamiltonian has a form:

$$
\begin{aligned}
& \dot{\rho}_{\mathcal{S}}\left(x, x^{\prime}, t\right)=-\frac{i}{\hbar}\left\langle x\left|\left[H_{\text {ren }}(t), \rho_{\mathcal{S}}\right]\right| x^{\prime}\right\rangle \\
& -\left(\gamma(t)\left(x-x^{\prime}\right)\left(\partial_{x}+\partial_{x^{\prime}}\right)-D(t)\left(x-x^{\prime}\right)^{2}\right) \rho_{\mathcal{S}}\left(x, x^{\prime}, t\right) \\
& -i f(t)\left(x-x^{\prime}\right)\left(\partial_{x}+\partial_{x^{\prime}}\right) \rho_{\mathcal{S}}\left(x, x^{\prime}, t\right) .
\end{aligned}
$$

The calculations leading to this master equation are nontrivial. They involve use of relations between the coefficients $b_{k}$ and $a_{i j}$. The final result leads to explicit formulae for its coefficients:

$$
\begin{gathered}
\Omega_{r e n}(t) / 2=b_{1} \dot{b}_{2} / b_{2}-\dot{b}_{1} \\
\gamma(t)=-b_{1}-\dot{b}_{2} / 2 b_{2} \\
D(t)=\quad \begin{array}{l}
\dot{a}_{11}-4 a_{11} b_{1} \\
+\dot{a}_{12} b_{1} / b_{3}-\dot{b}_{2}\left(2 a_{11}+a_{12} b_{1} / b_{3}\right) / b_{2}
\end{array} \\
2 f(t)=\dot{a}_{12} / b_{3}-\dot{b}_{2} a_{12} /\left(b_{2} b_{3}\right)-4 a_{11} .
\end{gathered}
$$

The fact that the exact master equation (5.21) is local in time for an arbitrary spectrum of the environment is remarkable. It was demonstrated by $\mathrm{Hu}, \mathrm{Paz}$, and Zhang (1992) following discussions carried out under more restrictive assumptions by Caldeira and Leggett (1983), Haake and Reibold (1985), Grabert, Schramm, and Ingold (1988), and Unruh and Zurek (1989). It depends on the linearity of the problem, that allows one to anticipate (Gaussian) form of the propagator.

The above derivation of the exact master equation used the method of Paz (1994) (see also Paz and Zurek, 2001). Explicit formulae for the time-dependent coefficients can be obtained when one focuses on the perturbative master equation. It can be derived ab initio (see Paz and Zurek, 2001) but can be also obtained from the above results by finding a perturbative solution to Eq. (5.17), and then substituting it in Eqs. $(5.22 a-5.25 a)$. The resulting master equation in the operator form is:

$$
\begin{aligned}
\dot{\rho}_{\mathcal{S}}= & -\frac{i}{\hbar}\left[H_{\mathcal{S}}+M \tilde{\Omega}(t)^{2} x^{2} / 2, \rho_{\mathcal{S}}\right]-\frac{i \gamma(t)}{\hbar}\left[x,\left\{p, \rho_{\mathcal{S}}\right\}\right] \\
& -D(t)\left[x,\left[x, \rho_{\mathcal{S}}\right]\right]-\frac{f(t)}{\hbar}\left[x,\left[p, \rho_{\mathcal{S}}\right]\right]
\end{aligned}
$$

Coefficients such as the frequency renormalization $\tilde{\Omega}$, the relaxation coefficient $\gamma(t)$, and the normal and anomalous diffusion coefficients $D(t)$ and $f(t)$ are given by:

$$
\tilde{\Omega}^{2}(t)=-\frac{2}{M} \int_{0}^{t} d s \cos (\Omega s) \eta(s)
$$

$$
\begin{array}{r}
\gamma(t)=\frac{2}{M \Omega} \int_{0}^{t} d s \sin (\Omega s) \eta(s) \\
D(t)=\frac{1}{\hbar} \int_{0}^{t} d s \cos (\Omega s) \nu(s)
\end{array}
$$

$$
f(t)=-\frac{1}{M \Omega} \int_{0}^{t} d s \sin (\Omega s) \eta(s)
$$

These coefficient can be made even more explicit when a convenient specific model for the spectral density:

$$
C(\omega)=2 M \gamma_{0} \frac{\omega}{\pi} \frac{\Gamma^{2}}{\Gamma^{2}+\omega^{2}}
$$

is adopted. Above, $\gamma_{0}$ characterizes the strength of the interaction, and $\Gamma$ is the high-frequency cutoff. Then:

$$
\begin{aligned}
& \tilde{\Omega}^{2}=-\frac{2 \gamma_{0} \Gamma^{3}}{\Gamma^{2}+\Omega^{2}}\left(1-\left(\cos \Omega t-\frac{\Omega}{\Gamma} \sin \Omega t\right) e^{-\Gamma t}\right) \\
& \gamma(t)=\frac{\gamma_{0} \Gamma^{2}}{\Gamma^{2}+\Omega^{2}}\left(1-\left(\cos \Omega t-\frac{\Gamma}{\Omega} \sin \Omega t\right) e^{-\Gamma t}\right)
\end{aligned}
$$

Note that both of these coefficients are initially zero. They grow to their asymptotic values on a timescale set by the inverse of the cutoff frequency $\Gamma$.

The two diffusion coefficients can be also studied, but it is more convenient to evaluate them numerically. In Fig. 5 we show their behavior. The normal diffusion coefficient quickly settles to its long-time asymptotic value:

$$
D_{\infty}=M \gamma_{0} \Omega \hbar^{-1} \operatorname{coth}(\hbar \Omega \beta / 2) \Gamma^{2} /\left(\Gamma^{2}+\Omega^{2}\right) .
$$

The anomalous diffusion coefficient $f(t)$ also approaches asymptotic value. For high temperature it is suppressed by a cutoff $\Gamma$ with respect to $D_{\infty}$, but the approach to $f_{\infty}$ is more gradual, algebraic rather than exponential. Environments with different spectral content exhibit different behavior (Hu, Paz, and Zhang, 1992; Paz, Habib, and Zurek, 1993; Paz, 1994; Anglin, Paz and Zurek, 1997).

\section{B. Decoherence in quantum Brownian motion}

The coefficients of the master equation we have just derived can be computed under a variety of different assumptions. The two obvious characteristics of the environment one can change are its temperature $T$ and its spectral density $C(\omega)$. One general conclusion: In case of high temperatures, $D(t)$ tends to a temperaturedependent constant, and dominates over $f(t)$. Indeed, in this case all of the coefficients settle to asymptotic values after an initial transient. Thus:

$$
\begin{aligned}
\dot{\rho}_{\mathcal{S}}= & -\frac{i}{\hbar}\left[H_{r e n}, \rho_{\mathcal{S}}\right]-\gamma\left(x-x^{\prime}\right)\left(\partial_{x}-\partial_{x^{\prime}}\right) \rho_{\mathcal{S}} \\
& -\frac{2 M \gamma k_{B} T}{\hbar^{2}}\left(x-x^{\prime}\right)^{2} \rho_{\mathcal{S}} .
\end{aligned}
$$


This master equation for $\rho\left(x, x^{\prime}\right)$ obtains in the unrealistic but convenient limit known as the high temperature approximation valid when $k_{B} T$ is much higher than all the other relevant energy scales, including the energy content of the initial state and the frequency cutoff in $C(\omega)$ (see, e.g, Caldeira and Leggett, 1983). However, when these restrictive conditions hold, Eq. (5.29) can be written for an arbitrary $V(x)$. To see why, we give a derivation patterned on $\mathrm{Hu}, \mathrm{Paz}$, and Zhang (1993).

We start with the propagator, Eq. (5.5), $\rho_{\mathcal{S}}\left(x, x^{\prime}, t\right)=$ $J\left(x, x^{\prime}, t \mid x_{0}, x_{0}^{\prime}, t_{0}\right) \rho_{\mathcal{S}}\left(x_{0}, x_{0}^{\prime}, t_{0}\right)$, which we shall treat as if it were an equation for state vector of the two dimensional system with coordinates $x, x^{\prime}$. The propagator is then given by the high-temperature version of Eq. (5.10);

$$
\begin{gathered}
J\left(x, x^{\prime}, t \mid x_{0}, x_{0}^{\prime}, t_{0}\right)=\int D x D x^{\prime} \exp \frac{i}{\hbar}\left\{I_{R}(x)-I_{R}\left(x^{\prime}\right)\right\} \\
e^{-M \gamma\left(\int_{0}^{t} d s\left[x \dot{x}-x^{\prime} \dot{x}^{\prime}+x \dot{x}^{\prime}-x^{\prime} \dot{x}\right]+\frac{2 k_{B} T}{\hbar^{2}}\left[x-x^{\prime}\right]^{2}\right)}
\end{gathered}
$$

The term in the exponent can be interpreted as an effective Lagrangian of a two-dimensional system:

$$
\begin{gathered}
L_{e f f}\left(x, x^{\prime}\right)=M \dot{x}^{2} / 2-V_{R}(x)-M \dot{x}^{2} / 2+V_{R}\left(x^{\prime}\right) \\
+\gamma\left(x-x^{\prime}\right)\left(\dot{x}+\dot{x}^{\prime}\right)+i \frac{2 M \gamma k_{B} T}{\hbar^{2}}\left(x-x^{\prime}\right)^{2} .
\end{gathered}
$$

One can readily obtain the corresponding Hamiltonian;

$$
H_{e f f}=\dot{x} \partial L_{e f f} / \partial \dot{x}+\dot{x}^{\prime} \partial L_{e f f} / \partial \dot{x}^{\prime}-L_{e f f} .
$$

Conjugate momenta $p=p_{x}=M \dot{x}+\gamma\left(x-x^{\prime}\right)$ and $p^{\prime}=$ $p_{x^{\prime}}=-M \dot{x}^{\prime}+\gamma\left(x-x^{\prime}\right)$ are used to express the kinetic term of $H_{\text {eff }}$. After evaluating $\dot{x}$ and $\dot{x}^{\prime}$ in terms of $p$ and $p^{\prime}$ in the expression for $H_{\text {eff }}$ one obtains:

$$
\begin{aligned}
H_{e f f}= & \left(p-\gamma\left(x-x^{\prime}\right)\right)^{2} / 2 M-\left(p^{\prime}-\gamma\left(x-x^{\prime}\right)\right)^{2} / 2 M \\
& +V(x)-V\left(x^{\prime}\right)-i 2 M \gamma k_{B} T\left(x^{\prime}-x\right)^{2} / \hbar^{2}(52)
\end{aligned}
$$

This expression yields the operator that generates the evolution of the density matrix, Eq. (5.29).

The coefficients of Eq. (5.21) approach their hightemperature values quickly (see Fig. 5). Already for $T$ well below what the rigorous derivation would demand high-temperature limit appears to be an excellent approximation. The discrepancy - manifested by symptoms such as some of the diagonal terms of $\rho_{\mathcal{S}}\left(x^{\prime}, x\right)$ assuming negative values when the evolutions starts from an initial state that is so sharply localized in position to have kinetic energy in excess of the values allowed by the high-temperature approximation - is limited to the initial instant of order $1 / \Gamma$, and is known to be essentially unphysical for other reasons (Unruh and Zurek, 1989; Ambegoakar, 1991; Romero and Paz, 1997; Anglin, Paz, and Zurek, 1997). This short - time anomaly is closely tied to the fact that Eq. (5.33) (and, indeed, many of the exact or approximate master equations derived to date) does not have the Lindblad form (Lindblad, 1976; see also Gorini, Kossakowski and Sudarshan, 1976, Alicki and Lendi, 1987) of a dynamical semigroup.
High temperature master equation (5.29) is a good approximation in a wider range of circumstances than the one for which it was derived (Feynman and Vernon, 1963; Dekker, 1977; Caldeira and Leggett, 1983). Moreover, our key qualitative conclusion - rapid decoherence in the macroscopic limit - does not crucially depend on the approximations leading to Eq. (5.29). We shall therefore use it in our further studies.

\section{Decoherence timescale}

In the macroscopic limit (that is, when $\hbar$ is small compared to other quantities with dimensions of action, such as $\sqrt{2 M k_{B} T\left\langle\left(x-x^{\prime}\right)^{2}\right\rangle}$ in the last term) the hightemperature master equation is dominated by:

$$
\partial_{t} \rho_{\mathcal{S}}\left(x, x^{\prime}, t\right)=-\gamma\left\{\frac{\left(x-x^{\prime}\right)}{\lambda_{T}}\right\}^{2} \rho_{\mathcal{S}}\left(x, x^{\prime}, t\right) .
$$

Above;

$$
\lambda_{T}=\frac{\hbar}{\sqrt{2 M k_{B} T}}
$$

is thermal de Broglie wavelength. Thus, the density matrix looses off-diagonal terms in position representation:

$$
\rho_{\mathcal{S}}\left(x, x^{\prime}, t\right)=\rho_{\mathcal{S}}\left(x, x^{\prime}, 0\right) e^{-\gamma t\left(\frac{x-x^{\prime}}{\lambda_{T}}\right)^{2}} .
$$

while the diagonal $\left(x=x^{\prime}\right)$ remains untouched.

Quantum coherence decays exponentially at the rate given by the relaxation rate times the square of the distance measured in units of thermal de Broglie wavelength (Zurek, 1984a). Position is the "instantaneous pointer observable": If Eq. (5.36) was always valid, eigenstates of position would attain the classical status.

The importance of position can be traced to the nature of the interaction Hamiltonian between the system and the environment. According to Eq. (5.3):

$$
H_{\mathcal{S E}}=x \sum_{n} c_{n} q_{n}
$$

This form of $H_{\mathcal{S E}}$ is motivated by physics (Zurek, 1982; 1991): Interactions depend on the distance. However, had we endeavored to find a situation where a different form of the interaction Hamiltonian - say, a momentumdependent interaction - was justified, the form and consequently predictions of the master equation would have been analogous to Eq. (5.36), but with a substitution of the relevant observable "monitored" by the environment for $x$. Such situations may be experimentally accessible (Poyatos, Cirac, and Zoller, 1996) providing a test of one of the key ideas of einselection: the relation between the form of interaction and the preferred basis.

The effect of the evolution, Eqs. (5.34) - (5.36), on the density matrix in the position representation is easy to envisage. Consider a superposition of two minimum 
uncertainty Gaussians. Off-diagonal peaks represent coherence. They decay on a decoherence timescale $\tau_{D}$, or with a decoherence rate (Zurek, 1984a, 1991);

$$
\tau_{D}^{-1}=\gamma\left(\frac{x-x^{\prime}}{\lambda_{T}}\right)^{2}
$$

Thermal de Broglie wavelength, $\lambda_{T}$, is microscopic for massive bodies and for the environments at reasonable temperatures. For a mass $1 \mathrm{~g}$ at room temperature and for the separation $x^{\prime}-x=1 \mathrm{~cm}$, Eq. (5.38) predicts decoherence approximately $10^{40}$ times faster than relaxation! Even cosmic microwave background suffices to cause rapid loss of quantum coherence in objects as small as dust grains (Joos and Zeh, 1985). These estimates for the rates of decoherence and relaxation should be taken with a grain of salt: Often the assumptions that have led to the simple high temperature master equation (5.29) are not valid (Gallis \& Fleming, 1990; Gallis, 1992; Anglin, Paz \& Zurek, 1997). For example, the decoherence rate cannot be faster than inverse of the spectral cutoff in Eq. (5.27), or than the rate with which the superposition is created. Moreover, for large separations quadratic dependence of decoherence rate may saturate (Gallis and Fleming, 1990; Anglin, Paz, and Zurek, 1997) as seen in the "simulated decoherence" experiments of Cheng and Raymer (1999). Nevertheless, in the macroscopic domain decoherence of widely delocalized "Schrödinger cat" states will occur very much faster than relaxation, which proceeds at the rate given by $\gamma$.

\section{Phase space view of decoherence}

A useful alternative way of illustrating decoherence is afforded by the Wigner function representation:

$$
W(x, p)=\frac{1}{2 \pi \hbar} \int_{-\infty}^{+\infty} d y e^{(i p y / \hbar)} \rho\left(x+\frac{y}{2}, x-\frac{y}{2}\right) .
$$

Evolution equation followed by the Wigner function obtains through the Wigner transform of the corresponding master equation. In the high temperature limit, Eq. (5.29) (valid for general potentials) this yields:

$$
\partial_{t} W=\left\{H_{r e n}, W\right\}_{M B}+2 \gamma \partial_{p}(p W)+D \partial_{p p} W .
$$

The first term - Moyal bracket - is the Wigner transform of the von Neumann equation (see Section III). In the linear case it reduces to the Poisson bracket. The second term is responsible for relaxation. The last diffusive term is responsible for decoherence.

Diffusion in momentum occurs at the rate set by $D=2 M \gamma k_{B} T$. Its origin can be traced to the continuous "measurement" of the position of the system by the environment: In accord with the Heisenberg indeterminacy, measurement of position results in the increase of the uncertainty in momentum (see Section IV).

Decoherence in phase space can be explained on the example of a superposition of two Gaussian wavepackets.
Wigner function in this case is given by:

$$
\begin{array}{r}
W(x, p)=G\left(x+x_{0}, p\right)+G\left(x-x_{0}, p\right) \\
+(\pi \hbar)^{-1} \exp \left(-p^{2} \xi^{2} / \hbar^{2}-x^{2} / \xi^{2}\right) \cos (\Delta x p / \hbar),
\end{array}
$$

where;

$$
G\left(x \pm x_{0}, p-p_{0}\right)=\frac{e^{-\left(x \mp x_{0}\right)^{2} / \xi^{2}-\left(p-p_{0}\right)^{2} \xi^{2} / \hbar^{2}}}{\pi \hbar} .
$$

We have assumed the Gaussians are not moving $\left(p_{0}=0\right)$.

The oscillatory term in Eq. (5.41) is the signature of superposition. The frequency of the oscillations is proportional to the distance between the peaks. When the separation is only in position $x$, this frequency is:

$$
f=\Delta x / \hbar=2 x_{0} / \hbar \text {. }
$$

Ridges and valleys of the interference pattern are parallel to the separation between the two peaks. This, and the fact that $\hbar$ appears in the interference term in $W$ is important for phase space derivation of the decoherence time. We focus on the dominant effect and direct our attention on the last term of Eq. (5.40). Its effect on the rapidly oscillating interference term will be very different from its effect on the two Gaussians: The interference term is dominated by the cosine:

$$
W_{\text {int }} \sim \cos \left(\frac{\Delta x}{\hbar} p\right)
$$

This is an eigenfunction of the diffusion operator. Decoherence timescale emerges (Zurek, 1991) from the corresponding eigenvalue;

$$
\dot{W}_{i n t} \approx-\left\{D \Delta x^{2} / \hbar^{2}\right\} \times W_{i n t} .
$$

We have recovered the formula for $\tau_{D}$, Eq. (5.38), from a different looking argument: Eq. (5.40) has no explicit dependence on $\hbar$ for linear potentials (in the nonlinear case $\hbar$ enters through the Moyal bracket). Yet, the decoherence timescale contains $\hbar$ explicitly $-\hbar$ enters through Eq. (5.43), that is through its role in determining the frequency of the interference pattern $W_{\text {int }}$.

The evolution of the pure initial state of the type considered here is shown in Fig. 6. There we illustrate evolution of the Wigner function for two initial pure states: Superposition of two positions and superposition of two momenta. There is a noticeable difference in the rate at which the interference term disappears between these two cases. This was anticipated. The interaction in Eq. (5.3) is a function of $x$. Therefore, $x$ is monitored by the environment directly, and the superposition of positions decoheres almost instantly. By contrast, the superposition of momenta is initially insensitive to the monitoring by the environment - the corresponding initial state is already well localized in the observable singled out by the interaction. However, a superposition of momenta leads to a superposition of positions, and hence to decoherence, albeit on a dynamical (rather than $\tau_{D}$ ) timescale. 
An intriguing example of a long-lived superposition of two seemingly distant Gaussians was pointed out by Braun, Braun and Haake (2000) in the context of superradiance. As they note, the relevant decohering interaction cannot distinguish between some such superpositions, leading to a 'Schrödinger cat' pointer subspace.

\section{Predictability sieve in phase space}

Decoherence rapidly destroys non-local superpositions. Obviously, states that survive must be localized. However, they cannot be localized to a point in $x$, as this would imply - by Heisenberg's indeterminacy - an infinite range in momenta, and, hence, of velocities. As a result, a wave function localized too well at one instant would become very non-local a moment later.

Einselected pointer states minimize the damage done by decoherence over the "timescale of interest" (usually associated with predictability or with dynamics). They can be found through the application of the predictability sieve outlined at the end of Section IV. To implement it, we compute entropy increase or purity loss for all initially pure states in the Hilbert space of the system under the cumulative evolution caused by the self-Hamiltonian and by the interaction with the environment. It would be a tall order to carry out the requisite calculations for an arbitrary quantum system interacting with a general environment. We focus on the exactly solvable case.

In the high temperature limit the master equations (5.26) and (5.29) can be expressed in the operator form:

$$
\begin{gathered}
\dot{\rho}=\frac{1}{i \hbar}\left[H_{r e n}, \rho\right]+\frac{\gamma}{i \hbar}[\{p, x\}, \rho]-\frac{\eta k_{B} T}{\hbar^{2}}[x,[x, \rho]] \\
-\frac{i \gamma}{\hbar}([x, \rho p]-[p, \rho x])
\end{gathered}
$$

Above, $\eta=2 M \gamma$ is the viscosity. Only the last two terms can change entropy. Terms of the form:

$$
\dot{\rho}=[\hat{O}, \rho]
$$

where $\hat{O}$ is Hermitean leave the purity $\varsigma=\operatorname{Tr} \rho^{2}$ and the von Neumann entropy $H=-\operatorname{Tr} \rho \lg \rho$ unaffected. This follows from the cyclic property of the trace:

$$
\frac{d}{d t} \operatorname{Tr} \rho^{N}=\sum_{k=1}^{N}\left(\operatorname{Tr} \rho^{k-1}[\hat{O} \rho] \rho^{N-k}\right)=0
$$

Constancy of $\operatorname{Tr} \rho^{2}$ is obvious, while for $\operatorname{Tr} \rho \lg \rho$ it follows when the logarithm is expanded in powers of $\rho$.

Equation (5.46) leads to the loss of purity at the rate (Zurek, 1993a):

$$
\frac{d}{d t} \operatorname{Tr} \rho^{2}=-\frac{4 \eta k_{B} T}{\hbar^{2}} \operatorname{Tr}\left(\rho^{2} x^{2}-(\rho x)^{2}\right)+2 \gamma \operatorname{Tr} \rho^{2}
$$

The second term increases purity - decreases entropy - as the system is damped from an initial highly mixed state.
For predictability sieve this term is usually unimportant, as for a vast majority of the initially pure states its effect will be negligible when compared to the first, decoherence - related term. Thus, in the case of pure initial states:

$$
\frac{d}{d t} \operatorname{Tr} \rho^{2}=-\frac{4 \eta k_{B} T}{\hbar^{2}}\left(\left\langle x^{2}\right\rangle-\langle x\rangle^{2}\right),
$$

Therefore, the instantaneous loss of purity is minimized for perfectly localized states (Zurek, 1993a). The second term of Eq. (5.49) allows for the equilibrium. (Nevertheless, early on, and for very localized states, its presence causes an (unphysical) increase of purity to above unity. This is a well-known artifact of the high-temperature approximation (see discussion following Eq. (5.33)).

To find most predictable states relevant for dynamics consider entropy increase over a period of the oscillator. For a harmonic oscillator with mass $M$ and frequency $\Omega$, one can compute purity loss averaged over $\tau=2 \pi / \Omega$ :

$$
\left.\Delta \varsigma\right|_{0} ^{2 \pi / \Omega}=-2 D\left(\Delta x^{2}+\Delta p^{2} /(M \Omega)^{2}\right) .
$$

Above, $\Delta x$ and $\Delta p$ are dispersions of the state at the initial time. By the Heisenberg indeterminacy, $\Delta x \Delta p \geq$ $\hbar / 2$. The loss of purity will be smallest when:

$$
\Delta x^{2}=\hbar / 2 M \Omega, \Delta p^{2}=\hbar M \Omega / 2 .
$$

Coherent quantum states are selected by the predictability sieve in an underdamped harmonic oscillator (Zurek, 1993a; Zurek, Habib and Paz, 1993; Gallis, 1996; Tegmark and Shapiro, 1994; Wiseman and Vaccaro, 1998; Paraoanu, 2002). Rotation induced by the selfHamiltonian turns preference for states localized in position into preference for localization in phase space. This is illustrated in Fig. 7.

We conclude that for an underdamped harmonic oscillator coherent Gaussians are the best quantum theory has to offer as an approximation to a classical point. Similar localization in phase space should obtain in the reversible classical limit in which the familiar symptoms of the "openness" of the system - such as the finite relaxation rate $\gamma=\eta / 2 M-$ become vanishingly small. This limit can be attained for large mass $M \longrightarrow \infty$, while the viscosity $\eta$ remains fixed and sufficiently large to assure localization (Zurek, 1991; 1993a). This is, of course, not the only possible situation. Haake and Walls (1987) discussed the overdamped case, where pointer states are still localized, but relatively more narrow in position. On the other hand, "adiabatic" environment enforces einselection in energy eigenstates (Paz and Zurek, 1999).

\section{Classical limit in phase space}

There are three strategies that allow one to simultaneously recover both the classical phase space structure and the classical equations of motion. 


\section{Mathematical approach $(\hbar \rightarrow 0)$}

This "mathematical" classical limit could not be implemented without decoherence, since the oscillatory terms associated with interference do not have an analytic $\hbar \rightarrow 0$ limit (see, e.g, Peres, 1993). However, in the presence of the environment, the relevant terms in the master equations increase as $\mathcal{O}\left(\hbar^{-2}\right)$, and make the nonanalytic manifestations of interference disappear. Thus, phase space distributions can be always represented by localized coherent state "points", or by the distributions over the basis consisting of such points.

This strategy is easiest to implement starting from the phase-space formulation. It follows from Eq. (5.45) that the interference term in Eq. (5.41) will decay (Paz, Habib, and Zurek, 1993) over the time interval $\Delta t$ as:

$$
W_{i n t} \sim \exp \left(-\Delta t \frac{D \Delta x^{2}}{\hbar^{2}}\right) \cos \left(\frac{\Delta x}{\hbar} p\right) .
$$

As long as $\Delta t$ is large compared to decoherence timescale $\tau_{D} \simeq \hbar^{2} / D \Delta x^{2}$, the oscillatory contributions to the Wigner function $W(x, p)$ shall disappear with $\hbar \rightarrow 0$. Simultaneously, Gaussians representing likely locations of the system become narrower, approaching $\delta$-functions in phase space. For instance, in Eq. (5.42);

$$
\lim _{\hbar \rightarrow 0} G\left(x-x_{0}, p-p_{0}\right)=\delta\left(x-x_{0}, p-p_{0}\right)
$$

providing half-widths of the coherent states in $x$ and $p$ decrease to zero as $\hbar \rightarrow 0$. This would be assured when, for instance, in Eqs. (5.41)-(5.42):

$$
\xi^{2} \sim \hbar
$$

Thus, individual coherent-state Gaussians approach phase space points. This behavior indicates that in a macroscopic open system nothing but probability distributions over localized phase space points can survive in the $\hbar \rightarrow 0$ limit for any time of dynamical or predictive significance. (Coherence between immediately adjacent points separated only by $\sim \xi$, Eq. (5.55), can last longer. This is no threat to the classical limit. Small scale coherence is a part of a "quantum halo" of the classical pointer states (Anglin and Zurek, 1996).)

The mathematical classical limit implemented by letting $\hbar \rightarrow 0$ becomes possible in presence of decoherence. It is tempting to take this strategy to its logical conclusion, and represent every probability density in phase space in the point(er) basis of narrowing coherent states. Such a program is beyond the scope of this review, but the reader should be by now convinced that it is possible. Indeed, Perelomov (1986) shows that a general quantum state can be represented in a sparse basis of coherent states that occupy sites of a regular lattice, providing that the volume per coherent state "point" is no more than $(2 \pi \hbar)^{d}$ in the $d$-dimensional configuration space. In presence of decoherence arising from the coordinatedependent interaction, evolution of a general quantum superposition should be - after a few decoherence times - well approximated by the probability distribution over such Gaussian "points”.

\section{Physical approach: The macroscopic limit}

The possibility of the $\hbar \rightarrow 0$ classical limit in presence of decoherence is of interest. But $\hbar=1.05459 \times$ $10^{-27}$ erg s. Therefore, a physically more reasonable approach increases the size of the object, and, hence, its susceptibility to decoherence. This strategy can be implemented starting with Eq. (5.40). Reversible dynamics obtains as $\gamma \rightarrow 0$ while $D=2 M \gamma k_{B} T=\eta k_{B} T$ increases.

The decrease of $\gamma$ and the simultaneous increase of $\eta k_{B} T$ can be anticipated with the increase of the size and mass. Assume that density of the object is independent of its size $R$, and that the environment quanta scatter from its surface (as would photons or air molecules). Then $M \sim R^{3}$ and $\eta \sim R^{2}$. Hence:

$$
\begin{gathered}
\eta \sim \mathcal{O}\left(R^{2}\right) \longrightarrow \infty, \\
\gamma=\eta / 2 M \sim \mathcal{O}(1 / R) \longrightarrow 0,
\end{gathered}
$$

as $R \rightarrow \infty$ : Localization in phase space and reversibility can be simultaneously achieved in a macroscopic limit.

Existence of macroscopic classical limit in simple cases has been pointed out some time ago (Zurek 1984a, 1991; Gell-Mann and Hartle, 1993). We shall analyze it in the next section in a more complicated chaotic setting, where reversibility can no longer be taken for granted. In the harmonic oscillator case approximate reversibility is effectively guaranteed, as the action associated with the 1- $\sigma$ contour of the Gaussian state increases with time at the rate (Zurek, Habib, and Paz, 1993):

$$
\dot{I}=\gamma \frac{k_{B} T}{\hbar \Omega}
$$

Action $I$ is a measure of the lack of information about phase space location. Hence, its rate of increase is a measure of the rate of predictability loss. Trajectory is a limit of the "tube" swept in phase space by the moving volume representing instantaneous uncertainty of the observer about the state of the system. Evolution is approximately deterministic when the area of this contour is nearly constant. In accord with Eqs. (5.56)-(5.57) $\dot{I}$ tends to zero in the reversible macroscopic limit:

$$
\dot{I} \sim \mathcal{O}(1 / R)
$$

The existence of an approximately reversible trajectorylike thin tubes provides an assurance that, having localized the system within a regular phase space volume at $t=0$, we can expect that it can be found later inside the Liouville - transported contour of nearly the same measure. Similar conclusions follow for integrable systems. 


\section{Ignorance inspires confidence in classicality}

Dynamical reversibility can be achieved with einselection in the macroscopic limit. Moreover, $\dot{I} / I$ or other measures of predictability loss decrease with the increase of $I$. This is especially dramatic when quantified in terms of the von Neumann entropy, that, for Gaussian states, increases at the rate (Zurek, Habib and Paz, 1993):

$$
\dot{H}=\dot{I} \lg \frac{I+1}{I-1}
$$

The resulting $\dot{H}$ is infinite for pure coherent states $(I=$ 1 ), but quickly decreases with increasing $I$. Similarly, the rate of purity loss for Gaussians is:

$$
\dot{\varsigma}=\dot{I} / I^{2}
$$

Again, it tapers off for more mixed states.

This behavior is reassuring. It leads us to conclude that irreversibility quantified through, say, von Neumann entropy production, Eq. (5.60a), will approach $\dot{H} \approx 2 \dot{I} / I$, vanishing in the limit of large $I$. When in the spirit of the macroscopic limit we do not insist on the maximal resolution allowed by the quantum indeterminacy, the subsequent predictability losses measured by the increase of entropy or through the loss of purity will diminish. Illusions of reversibility, determinism, and exact classical predictability become easier to maintain in presence of ignorance about the initial state!

To think about phase space points one may not even need to invoke a specific quantum state. Rather, a point can be regarded as a limit of an abstract recursive procedure, in which phase space coordinates of the system are determined better and better in a succession of increasingly accurate measurements. One may be tempted to extrapolate this limiting process ad infintessimum which would lead beyond Heisenberg's indeterminacy principle and to a false conclusion that idealized points and trajectories "exist objectively", and that the insider view of Section II can be always justified. While in our quantum Universe this conclusion is wrong, and the extrapolation described above illegal, the presence, within the Hilbert space, of localized wavepackets near the minimum uncertainty end of such imagined sequences of measurements is reassuring. Ultimately, the ability to represent motion in terms of points and their time - ordered sequences (trajectories) is the essence of classical mechanics.

\section{E. Decoherence, chaos, and the Second Law}

Breakdown of correspondence in this chaotic setting was described in section III. It is anticipated to occur in all non-linear systems, as the stretching of the wavepacket by the dynamics is a generic feature, absent only in a harmonic oscillator. However, exponential instability of chaotic dynamics implies rapid loss of quantum - classical correspondence after the Ehrenfest time,
$t_{\hbar}=\Lambda^{-1} \ln \chi \Delta p / \hbar$. Here $\Lambda$ is the Lyapunov exponent, $\chi=\sqrt{V_{x} / V_{x x x}}$ typically characterizes the dominant scale of nonlinearities in the potential $V(x)$, and $\Delta p$ gives the coherence scale in the initial wavepacket. The above estimate, Eq. (3.5) depends on the initial conditions. It is smaller than, but typically close to, $t_{r}=\Lambda^{-1} \ln I / \hbar$, Eq. (3.6), where $I$ is the characteristic action of the system. By contrast, phase space patches of regular systems undergo stretching with a power of time. Consequently, loss of correspondence occurs only over a much longer $t_{r} \sim(I / \hbar)^{\alpha}$, that depends polynomially on $\hbar$.

\section{Restoration of correspondence}

Exponential instability spreads the wavepacket to a "paradoxical" extent at the rate given by the positive Lyapunov exponents $\Lambda_{+}^{(i)}$. Einselection attempts to enforce localization in phase space by tapering off interference terms at the rate given by the inverse of the decoherence timescale $\tau_{D}=\gamma^{-1}\left(\lambda_{T} / \Delta x\right)^{2}$. The two processes reach status quo when the coherence length $\ell_{c}$ of the wavepacket makes their rates comparable, that is:

$$
\tau_{D} \Lambda_{+} \simeq 1
$$

This yields an equation for the steady-state coherence length and for the corresponding momentum dispersion:

$$
\begin{gathered}
\ell_{c} \simeq \lambda_{T} \sqrt{\Lambda_{+} / 2 \gamma} ; \\
\sigma_{c}=\hbar / \ell_{c}=\sqrt{2 D / \Lambda_{+}} .
\end{gathered}
$$

Above, we have quoted results (Zurek and Paz, 1994) that follow from a more rigorous derivation of the coherence length $\ell_{c}$ than the "rough and ready" approach that led to Eq. (5.61). They embody the same physical argument, but seek asymptotic behavior of the Wigner function that evolves according to the equation:

$$
\begin{aligned}
\dot{W}= & \{H, W\}+\sum_{n \geq 1} \frac{\hbar^{2 n}(-)^{n}}{2^{2 n}(2 n+1) !} \partial_{x}^{2 n+1} V \partial_{p}^{2 n+1} W \\
& +D \partial_{p}^{2} W
\end{aligned}
$$

The classical Liouville evolution generated by Poisson bracket ceases to be a good approximation of the decohering quantum evolution when the leading quantum correction becomes comparable to the classical force:

$$
\frac{\hbar^{2}}{24} V_{x x x} W_{p p p} \approx \frac{\hbar^{2}}{24} \frac{V_{x}}{\chi^{2}} \frac{W_{p}}{\sigma_{c}^{2}}
$$

The term $\partial_{x} V \partial_{p} W$ represents the classical force in Poisson bracket. Quantum corrections are small when;

$$
\sigma_{c} \chi \gg \hbar
$$

Equivalently, Moyal bracket generates approximately Liouville flow when the coherence length satisfies:

$$
\ell_{c} \ll \chi
$$


This last inequality has an obvious interpretation: It is a demand for the localization to within a region $\ell_{c}$ small compared to the scale $\chi$ of the nonlinearities of the potential. When this condition holds, classical force will dominate over quantum corrections.

Restoration of correspondence is illustrated in Fig. 8 where Wigner functions are compared with classical probability distributions in a chaotic system. The difference between the classical and quantum expectation values in same chaotic system is shown in Fig. 9. Even relatively weak decoherence suppresses the discrepancy, helping reestablish the correspondence: $D=0.025$ translates through Eq. (5.62) into coherence over $\ell_{c} \simeq 0.3$, not much smaller than the nonlinearity scale $\chi \simeq 1$ for the investigated Hamiltonian of Fig. 8.

\section{Entropy production}

Irreversibility is the price for the restoration of quantum-classical correspondence in chaotic dynamics. It can be quantified through the entropy production rate. The simplest argument recognizes that decoherence restricts spatial coherence to $\ell_{c}$. Consequently, as the exponential instability stretches the size $L^{(i)}$ of the distribution in directions corresponding to the positive Lyapunov exponents $\Lambda_{+}^{(i)}, L^{(i)} \sim \exp \left(\Lambda_{+}^{(i)} t\right)$ the squeezing mandated by the Liouville theorem in the complementary directions corresponding to $\Lambda_{-}^{(i)}$ will halt at $\sigma_{c}^{(i)}$, Eq. (5.63). In this limit, the number of pure states needed to represent resulting mixture increases exponentially

$$
N^{(i)} \simeq L^{(i)} / \ell_{c}^{(i)}
$$

in each dimension. The least number of pure states overlapped by $W$ will be then $\mathcal{N}=\Pi_{i} N^{(i)}$. This implies:

$$
\dot{H} \simeq \partial_{t} \ln \mathcal{N} \simeq \sum_{i} \Lambda_{+}^{(i)} .
$$

This estimate for entropy production rate becomes accurate as the width of the Wigner function reaches saturation at $\sigma_{c}^{(i)}$. When a patch in phase space corresponding to the initial $W$ is regular and smooth on scales large compared to $\sigma_{c}^{(i)}$, evolution will start nearly reversibly (Zurek and Paz, 1994). However, as squeezing brings the extent of the effective support of $W$ close to $\sigma_{c}^{(i)}$, diffusion bounds from below the size of the smallest features of $W$. Stretching in the unstable directions continues unabated. As a consequence, the volume of the support of $W$ will grow exponentially, resulting in an entropy production rate set by Eq. (5.69), the sum of the classical Lyapunov exponents. Yet, it has an obviously quantum origin in decoherence. This quantum origin may be apparent initially, as the rate of Eq. (5.69) will be approached from above when the initial state is a non-local. On the other hand, in a multidimensional system different Lyapunov exponents may begin to contribute to entropy production at different instants (as the saturation condition,
Eq. (5.61), may not be met simultaneously for all $\left.\Lambda_{+}^{(i)}\right)$. Then the entropy production rate can accelerate (before subsiding as a consequence of approaching equilibrium).

The timescales on which this estimates of entropy production apply are still subject to investigation (Zurek and Paz, 1995a; Zurek, 1998b; Monteoliva and Paz 2000 \& 2001) and even controversy (Casati and Chirikov, 1995b; Zurek and Paz, 1995b). The instant when Eq. (5.69) becomes a good approximation corresponds to the moment when the exponentially unstable evolution forces the Wigner function to develop small phase space structures on the scale of the effective decoherence - imposed "coarse graining", Eq. (5.63). This will be a good approximation until the time $t_{E Q}$ at which equilibrium sets in. Both $t_{\hbar}$ have a logarithmic dependence on the corresponding (initial and equilibrium) phase space volumes $I_{0}$ and $I_{E Q}$, so the validity of Eq. (5.69) will be limited to $t_{E Q}-t_{\hbar} \simeq \Lambda^{-1} \ln I_{E Q} / I_{0}$.

There is a simple and conceptually appealing way to extend the interval over which entropy is produced at the rate given by Eq. (5.69): Imagine an observer monitoring a decohering chaotic system, finding out its state at time intervals small compared to $\Lambda^{-1}$, but large compared to the decoherence timescale. One can show (Zurek, 1998b) that the average increase of the size of the algorithmically compressed records of measurement of a decohering chaotic system (that is, the algorithmic randomness of the acquired data, see e.g. Cover and Thomas, 1991) is given - after conversion into bits from "nats" - by Eq. (5.69). This conclusion holds providing that the effect of the "collapses of the wavepacket" caused by the repeated measurements is negligible - i.e., the observer is "skillful". A possible strategy a skillful observer may adopt is that of indirect measurements, of monitoring a fraction of the environment responsible for decoherence to find out the state of the system. As we shall see in more detail in the following sections of the paper, this is a very natural strategy, often employed by the observers.

A classical analogue of Eq. (5.69) has been obtained by Kolmogorov (1960) and Sinai (1960) starting from very different, mathematical arguments that in effect relied on an arbitrary but fixed coarse graining imposed on phase space (see Wehrl, 1978). Decoherence leads to a similar looking quantum result in a very different fashion: Coarse graining is imposed by the coupling to the environment, but only in the sense implied by the einselection. Its "graininess" (resolution) is set by the accuracy of the monitoring environment. This is especially obvious when the indirect monitoring strategy mentioned immediately above is adopted by the observers. Preferred states will be partly localized in $x$ and $p$, but (in contrast to the harmonic oscillator case with its coherent states) details of this environment - imposed coarse graining will likely depend on phase space location, precise nature of the coupling to the environment, etc. Yet, in the appropriate limit, Eqs. (5.66)-(5.67), the asymptotic entropy production rate defined with the help of the algorithmic contribution discussed above (i.e., in a manner of the 
physical entropy, Zurek (1989)) does not depend on the strength or nature of the coupling, but is instead given by the sum of the positive Lyapunov exponents.

Von Neumann entropy production consistent with the above discussion has been now seen in numerical studies of decohering chaotic systems (Shiokawa and $\mathrm{Hu}$, 1995; Furuya, Nemes, and Pellegrino, 1998; Miller and Sarkar, 1999; Schack, 1998; Monteoliva and Paz, 2000 \& 2001). Extensions to situations where relaxation matters, as well as in the opposite direction - to where decoherence is relatively gentle - have been also discussed (Brun, Percival, and Schack, 1995; Miller, Sarkar, and Zarum, 1998; Pattanyak, 2000). An exciting development is the experimental study of the Loschmidt echo using NMR techiques (Levstein, Usaj, and Pastawski, 1998; Levstein et al, 2000, Jalabert and Pastawski, 2001) which sheds a new light on the irreversibility in decohering complex dynamical systems. We shall briefly return to this subject in Section VIII, while discussing experimental investigations relevant for decoherence and quantum chaos.

\section{Quantum predictability horizon}

The cross section $I$ of the trajectory-like tube containing the state of the harmonic oscillator in phase space increases only slowly, Eq. (5.58), at a rate which - once the limiting Gaussian is reached - does not depend on $I$. By contrast, in chaotic quantum systems this rate is:

$$
\dot{I} \simeq I \sum_{i} \Lambda_{+}^{(i)}
$$

A fixed rate of entropy production implies an exponential increase of the cross-section of the tube of, say, the 1$\sigma$ contour containing points consistent with the initial conditions: Phase space support expands exponentially.

This quantum view of chaotic evolution can be compared with the classical "deterministic chaos". In both cases, in the appropriate classical limit - which may involve either mathematical $\hbar \rightarrow 0$, or a macroscopic limit - the future state of the system can be in principle predicted to a set accuracy for an arbitrarily long time. However, such predictability can be accomplished only when the initial conditions are given with the resolution that increases exponentially with the time interval over which the predictions are to be valid. Given the fixed value of $\hbar$, there is therefore a quantum predictability horizon after which the Wigner function of the system starting from an initial minimum uncertainty Gaussian becomes stretched to the size of the order of the characteristic dimension of the system (Zurek, 1998b). The ability to predict the location of the system in the phase space is then lost after $t \sim t_{\hbar}$, Eq. (3.5), regardless of whether evolution is generated by the Poisson or Moyal bracket, or, indeed, whether the system is closed or open.

The case of regular systems is closer to the harmonic oscillator. The rate of increase of the cross-section of phase space "trajectory tube" consistent with the initial patch in the phase space will asymptote to $\dot{I} \simeq$ const:

$$
\dot{H}=\dot{I} / I \sim 1 / t
$$

Thus, initial conditions allow one to predict future of a regular system for time intervals that are exponentially longer than in the chaotic case. The rate of entropy production of an open quantum system is therefore a very good indicator of the nature of its dynamics, as was conjectured some time ago (Zurek and Paz, 1995a), and as seems born out in the numerical simulations (Shiokawa and Hu, 1995; Miller, Sarkar and Zarum, 1998; Miller and Sarkar, 1999; Monteoliva and Paz, 2000 \& 2001).

\section{EINSELECTION AND MEASUREMENTS}

It is often said that quantum states play only an epistemological role in describing observers knowledge about the past measurement outcomes that have prepared the system (Jammer, 1974; d'Espagnat, 1976, 1995; Fuchs and Peres, 2000). In particular - and this is a key argument against their objective existence (against their ontological status) - it is impossible to find out what the state of an isolated quantum system is without prior information about the observables used to prepare it: Measurement of observables that do not commute with this original set will inevitably create a different state.

The incessant monitoring of the einselected observables by the environment allows pointer states to exist in much the same way classical states do. This ontological role of the einselected quantum states can be justified operationally, by showing that in presence of einselection one can find out what the quantum state is, without inevitably re-preparing it by the measurement. Thus, einselected quantum states are no longer just epistemological. In a system monitored by the environment what is - the einselected states - coincides with what is known to be-what is recorded by the environment (Zurek, 1993a,b; 1998a).

The conflict between the quantum and the classical was originally noted and discussed almost exclusively in the context of quantum measurements (Bohr, 1928; Mott, 1929; von Neumann, 1932; Dirac, 1947; Zeh, 1971, 1973, 1993; d'Espagnat, 1976, 1995; Zurek 1981, 1982, 1983, 1991, 1993a\&b, 1998a; Omnès 1992, 1994; Elby, 1993, 1998; Butterfield, 1996; Donald, 1995; Giulini et al., 1996; Bub, 1997; Saunders, 1998; Healey, 1998; Bacciagaluppi and Hemmo, 1998; Healey and Hellman, 1998). Here I shall consider quantum measurements, and, more to the point, acquisition of information in quantum theory from the point of view of decoherence and einselection.

\section{A. Objective existence of einselected states}

To demonstrate objective existence of einselected states we now develop an operational definition of existence and show how, in the open system, one can find out what the 
state was and is, rather than just "prepare" it. This point was made before (Zurek, 1993a; 1998a), but this is first time I discuss it in more detail.

Objective existence of states can be defined operationally by considering two observers. The first of them is the record keeper $\mathbf{R}$. He prepares the states with the original measurement and will use his records to find out if they were disturbed by the measurements carried out by other observers, e.g. the spy $\mathbf{S}$. The goal of $\mathbf{S}$ is to discover the state of the system without perturbing it. When an observer can consistently find out the state of the system without changing it, that state - by our operational definition - will be said to exist objectively.

In absence of einselection the situation of the spy $\mathbf{S}$ is hopeless: $\mathbf{R}$ prepares states by measuring sets of commuting observables. Unless $\mathbf{S}$ picks, by sheer luck, the same observables in case of each state, his measurements will re-prepare states of the systems. Thus, when $\mathbf{R}$ remeasures using the original observables, he will likely find answers different from his records. The spy $\mathbf{S}$ will "get caught", because it is impossible to find out an initially unknown state of an isolated quantum system.

In presence of environmental monitoring the nature of the "game" between $\mathbf{R}$ and $\mathbf{S}$ is dramatically altered. Now it is no longer possible for $\mathbf{R}$ to prepare an arbitrary pure state that will persist or predictably evolve without losing purity. Only the einselected states that are already monitored by the environment - that are selected by the predictability sieve - will survive. By the same token, $\mathbf{S}$ is no longer clueless about the observables he can measure without leaving incriminating evidence. For example, he can independently prepare and test survival of various states in the same environment to establish which states are einselected, and then measure appropriate pointer observables. Better yet, $\mathbf{S}$ can forgo direct measurements of the system, and gather information indirectly, by monitoring the environment.

This last strategy may seem contrived, but indirect measurements - acquisition of information about the system by examining fragments of the environment that have interacted with it - is in fact more or less the only strategy employed by the observers. Our eyes, for example, intercept only a small fraction of the photons that scatter from various objects. The rest of the photons constitute the environment, which retains at least as complete a record of the same einselected observables as we can obtain (Zurek, 1993; 1998a).

The environment $\mathcal{E}$ acts as a persistent observer, dominating the game with frequent questions, always about the same observables, compelling both $\mathbf{R}$ and $\mathbf{S}$ to focus on the einselected states. Moreover, $\mathcal{E}$ can be persuaded to share its records of the system. This accessibility of the einselected states is not a violation of the basic tenets of quantum physics. Rather, it is a consequence of the fact that the data required to turn quantum state into an ontological entity - an einselected pointer state - are abundantly supplied by the environment.

We emphasize the operational nature of this criterion for existence: There may be in principle a pure state of the Universe including the environment, the observer, and the measured system. While this may matter to some (Zeh, 2000), real observers are forced to perceive the Universe the way we do: We are a part of the Universe, observing it from within. Hence, for us, environmentinduced superselection specifies what exists.

Predictability emerges as a key criterion of existence. The only states $\mathbf{R}$ can rely on to store the information are the pointer states. They are also the obvious choice for $\mathbf{S}$ to measure. Such measurements can be accomplished without danger of re-preparation. Einselected states are insensitive to measurement of the pointer observables they have already been "measured" by the environment. Therefore, additional projections $P_{i}$ onto the einselected basis will not perturb the density matrix (Zurek, 1993a) - it will be the same before and after the measurement:

$$
\rho_{\text {after }}^{D}=\sum_{i} P_{i} \rho_{\text {before }}^{D} P_{i} .
$$

Correlations with the einselected states will be left intact (Zurek 1981; 1982).

Superselection for the observable $\hat{A}=\sum_{i} \lambda_{i} P_{i}$ with essentially arbitrary non-degenerate eigenvalues $\lambda_{i}$ and eigenspaces $P_{i}$ can be expressed (Bogolubov et al., 1990) through Eq. (6.1). Einselection attains this, guaranteeing diagonality of density matrices in the projectors $P_{i}$ corresponding to pointer states. These are sometimes called decoherence - free subspaces when they are degenerate; compare also nonabelian case of noiseless subsystems discussed in quantum computation; see Zanardi and Rasetti, 1997; Zanardi 1998; Duan and Guo 1998; Lidar, Bacon, and Whaley, 1999; Knill, Laflamme, and Viola, 2000; Blanchard and Olkiewicz, 2000; Zanardi 2000).

\section{B. Measurements and memories}

The memory of an apparatus or of an observer can be modelled as an open quantum $\mathcal{A}$, interacting with $\mathcal{S}$ through a Hamiltonian explicitly proportional to the measured observable $\hat{s}^{5}$

$$
H_{\text {int }}=-g \hat{s} \hat{B} \sim \hat{s} \frac{\partial}{\partial \hat{A}} .
$$

Von Neumann (1932) considered an apparatus isolated from the environment. At the instant of the interaction

\footnotetext{
5 The observable $\hat{s}$ of the system and $\hat{B}$ of the apparatus memory need not be discrete with a simple spectrum as was previously assumed. Even when $\hat{s}$ has a complicated spectrum, the outcome of the measurement can be recorded in the eigenstates of the memory observable $\hat{A}$, conjugate of $\hat{B}$, Eq. (2.21). For the case of discrete $\hat{s}$ the necessary calculations that attain premeasurement - the quantum correlation that is the first step in the creation of the record - were already carried out in section II. For the other situations they are quite similar. In either case, they follow the general outline of the von Neumann's (1932) discussion.
} 
between the apparatus and the measured system this is a convenient assumption. For us it suffices to assume that, at that instant, the interaction Hamiltonian between the system and the apparatus dominates. This can be accomplished by taking the coupling $g$ in Eq. (6.2) to be $g(t) \sim \delta\left(t-t_{0}\right)$. Premeasurement happens at $t_{0}:$

$$
\left(\sum_{i} \alpha_{i}\left|s_{i}\right\rangle\right)\left|A_{0}\right\rangle \longrightarrow \sum_{i} \alpha_{i}\left|s_{i}\right\rangle\left|A_{i}\right\rangle
$$

In practice the action is usually large enough to accomplish amplification. As we have seen in section II, all this can be done without an appeal to the environment.

For a real apparatus interaction with the environment is inevitable. Idealized effectively classical memory will retain correlations, but will be subject to einselection: Only the einselected memory states (rather than their superpositions) will be useful for (or, for that matter, accessible to) the observer: Decoherence timescale is very short compared to the time after which memory states are typically consulted (i. e., copied or used in information processing), which is in turn much shorter than the relaxation timescale, on which memory "forgets".

Decoherence leads to classical correlation:

$$
\begin{aligned}
& \rho_{\mathcal{S} \mathcal{A}}^{P}=\sum_{i, j} \alpha_{i} \alpha_{j}^{*}\left|s_{i}\right\rangle\left\langle s_{j}|| A_{i}\right\rangle\left\langle A_{j}\right| \longrightarrow \\
& \longrightarrow \sum_{i}\left|\alpha_{i}\right|^{2}\left|s_{i}\right\rangle\left\langle s_{i}|| A_{i}\right\rangle\left\langle A_{i}\right|=\rho_{\mathcal{S} \mathcal{A}}^{D}
\end{aligned}
$$

following an entangling premeasurement. Left hand side of Eq. (6.4) coincides with Eq. (2.44c), the "outsiders" view of the classical measurement. We shall see how and to what extent its other aspects - including the insiders Eq. (2.44a) and the discoverers Eq. (2.44b) - can be understood through einselection.

\section{Axioms of quantum measurement theory}

Our goal is to establish whether the above model can fulfill requirements expected from measurement in textbooks (that are, essentially without exception, written in the spirit of the Copenhagen Interpretation). There are several equivalent 'textbook' formulations of axioms of quantum theory. We shall (approximately) follow Farhi, Goldstone, and Gutmann (1989) and posit:

(i) The states of a quantum system $\mathcal{S}$ are associated with the vectors $|\psi\rangle$ which are the elements of the Hilbert space $\mathcal{H}_{\mathcal{S}}$ that describes $\mathcal{S}$.

(ii) The states evolve according to $i \hbar|\dot{\psi}\rangle=H|\psi\rangle$ where $H$ is Hermitean.

(iii a) Every observable $O$ is associated with a Hermitian operator $\hat{O}$.

(iii b) The only possible outcome of a measurement of $O$ is an eigenvalue $o_{i}$ of $\hat{O}$. (iv) Immediately after a measurement that yields the value $o_{i}$ the system is in the eigenstate $\left|o_{i}\right\rangle$ of $\hat{O}$.

(v) If the system is in a normalized state $|\psi\rangle$, then a measurement of $\hat{O}$ will yield the value $o_{i}$ with the probability $p_{i}=\left|\left\langle o_{i} \mid \psi\right\rangle\right|^{2}$.

The first two axioms make no reference to measurements. They state the formalism of the theory. Axioms (iii) - (v) are, on the other hand, at the heart of the present discussion. In the spirit, they go back to Bohr and Born. In the letter, they follow von Neumann (1932) and Dirac (1947). The two key issues are the projection postulate, implied by a combination of (iv) with (iiib), and the (Born's) probability interpretation, axiom (v).

To establish (iiib), (iv) and (v) we shall interpret - in operational terms - statements such as "the system is in the eigenstate" and "measurement will yield value ... with the probability ..." by specifying what these statements mean for the sequences of records made and maintained by an idealized, but physical memory.

We note that the above Copenhagen-like axioms presume existence of quantum systems and of classical measuring devices. This (unstated) "axiom (o)" complements axioms (i) - (v). Our version of axiom (o) demands that the Universe consists of systems, and asserts that a composite system can be described by a tensor product of the Hilbert spaces of the constituent systems. Some quantum systems can be measured, others can be used as measuring devices and/or memories, and as quantum environments that interact with either or both.

Axioms (iii) - (v) contain many idealizations. For instance, in real life or in laboratory practice measurements have errors (and hence can yield outcomes other than the eigenvalues $o_{i}$ ). Moreover, only rarely do they "prepare" the system in the eigenstate of the observable they are designed to measure. Furthermore, coherent states - often an outcome of measurements, e. g., in quantum optics - form an overcomplete basis. Thus, their detection does not correspond to a measurement of a Hermitean observable. Last not least, the measured quantity may be inferred from some other quantity (e.g. deflection of a beam in the Stern-Gerlach experiment). Yet, we shall not go beyond the idealizations of (i) - (v) above. Our goal is to describe measurements in a quantum theory "without collapse", to use axioms (o), (i) and (ii) to understand the origin of the other axioms. Non-ideal measurements are a fact of life incidental to this goal.

\section{Observables are Hermitean - axiom (iiia)}

In the model of measurement considered here the observables are Hermitean as a consequence of the assumed premeasurement interaction, e.g. Eq. (2.24). In particular, $H_{\text {int }}$ is a product of the to-be-measured observable of the system and of the "shift operator" in the pointer of the apparatus or in the record state of the 
memory. Interactions involving non-Hermitean operators (e.g., $H_{\text {int }} \sim a^{\dagger} b+a b^{\dagger}$ ) may be, however, also considered.

It is tempting to speculate that one could dispose of the observables (and, hence, of the postulate (iiia)) altogether in the formulation of the axioms of quantum theory. The only ingredients necessary to describe measurements are then the effectively classical, but ultimately quantum apparatus, and the measured system. Observables emerge as a derived concept, as a useful idealization, ultimately based on the structure of the Hamiltonians. Their utility relies on the conservation laws that relate outcomes of several measurements. Most basic of these laws states that the system that did not (have time to) evolve will be found in the same state when it is remeasured: This is the content of axiom (iv). Other conservation laws are also reflected in the patterns of correlation in the measurement records, that must in turn arise from the underlying symmetries of the Hamiltonians.

Einselection should be included in this program, as it decides which of the observables are accessible and useful - which are effectively classical. It is conceivable that also the "fundamental" superselection may emerge in this manner (see Zeh, 1970; Zurek, 1982; for early speculations; Giulini, Kiefer, and Zeh, 1995; and Kiefer, 1996; Giulini, 2000, for the present status of this idea).

\section{Eigenvalues as outcomes - axiom (iiib)}

This statement is the first part of the "collapse" postulate. Given einselection, (iiib) is easy to justify: We need to show that only the records inscribed in the einselected states of the apparatus pointer can be read off, and that in a well-designed measurement - they correlate with the eigenstates (and, therefore, eigenvalues) of the measured observable $\hat{s}$.

With Dirac (1947) and von Neumann (1932) we assume that the apparatus is built so that it satisfies the obvious "truth table" when the eigenstates of the measured observable are at the input:

$$
\left|s_{i}\right\rangle\left|A_{0}\right\rangle \longrightarrow\left|s_{i}\right\rangle\left|A_{i}\right\rangle
$$

To assure this one can implement the interaction in accord with Eq. (6.2) and the relevant discussion in section II. This is not to say that there are no other ways: Braginski and Khalili (1996), Aharonov, Anandan and Vaidman (1993), and Unruh (1994) have all considered "adiabatic measurements", that correlate the apparatus with the discrete energy eigenstates of the measured system, nearly independently of the structure of $H_{\text {int }}$.

Truth table of Eq. (6.5) does not require collapse - for any initial $\left|s_{i}\right\rangle$ it represents a "classical measurement in quantum notation" in the sense of Section II. However, Eq. (6.5) typically leads to a superposition of outcomes. This is the "measurement problem". To address it, we assume that the record states $\left\{\left|A_{i}\right\rangle\right\}$ are einselected. This has two related consequences: (i) Following the measurement, the joint density matrix of the system and the apparatus decoheres, Eq. (6.3), so that it satisfies the superselection condition, Eq. (6.1), for $P_{i}=\left|A_{i}\right\rangle\left\langle A_{i}\right|$. (ii) Einselection restricts states that can be read of "as if they were classical" to pointer states.

Indeed, following decoherence only pointer states $\left\{\left|A_{i}\right\rangle\right\}$ of the memory can be measured without diminishing the correlation with the states of the system. Without decoherence, as we have seen in section II, one could use entanglement between $\mathcal{S}$ and $\mathcal{A}$ to end up with an almost arbitrary superposition states of either, and, hence, to violate the letter and the spirit of (iiib).

Outcomes are restricted to the eigenvalues of measured observables because of einselection. Axiom (iiib) is then a consequence of the effective classicality of pointer states, the only ones that can be "found out" without being disturbed. They can be consulted repeatedly and remain unaffected under the joint scrutiny of the observers and of the environment (Zurek, 1981; 1993a; 1998a).

\section{Immediate repeatability, axiom (iv)}

This axiom supplies the second half of the "collapse" postulate. It asserts that in the absence of (the time for) evolution quantum system will remain in the same state, and its re-measurement will lead to the same outcome. Hence, once the system is found out to be in a certain state, it really is there. As in Eq. (2.44b) observer perceives potential options "collapse" to a single actual outcome. (The association of the axiom (iv) with the collapse advocated here seems obvious, but it is not common: Rather, some form of our axiom (iiib) is usually regarded as the sole "collapse postulate".)

Immediate repeatability for Hermitean observables with discrete spectra is straightforward to justify on the basis of Schrödinger evolution generated by $H_{\text {int }}$ of Eq. (6.2) alone, although its implications depend on whether the premeasurement is followed by einselection. Everett (1957) used the "no decoherence" version as a foundation of his relative state interpretation. On the other hand, without decoherence and einselection one could postpone the choice of what was actually recorded by taking advantage of the entanglement between the system and the apparatus, evident on the left hand side of Eq. (6.3). For instance, a measurement carried out on the apparatus in a basis different from $\left\{\left|A_{i}\right\rangle\right\}$ would also exhibit a one-to-one correlation with the system: $\sum_{i} \alpha_{i}\left|s_{i}\right\rangle\left|A_{i}\right\rangle=\sum_{k} \beta_{k}\left|r_{k}\right\rangle\left|B_{k}\right\rangle$. This flexibility to rewrite wavefunctions in different bases comes at a price of relaxing the demand that the outcome states $\left\{\left|r_{k}\right\rangle\right\}$ be orthogonal (so that there would be no associated Hermitean observable). However, as was already noted, coherent states associated with a non-Hermitean annihilation operator can also be an outcome of a measurement. Therefore (and in spite of the strict interpretation of axiom (iiia)) this is not a very serious restriction.

In presence of einselection the basis ambiguity disappears. Immediate repeatability would apply only to the 
records made in the einselected states. Other apparatus observables lose correlation with the state of the system on decoherence timescale. In the effectively classical limit it is natural to demand repeatability extending beyond that very short time interval. This demand makes the role of einselection in establishing axiom (iv) evident. Indeed, such repeatability is - albeit in a more general context - the motivation for the predictability sieve.

\section{Probabilities, einselection and records}

Density matrix alone - without the preferred set of states - does not suffice as a foundation for a probability interpretation. For, any mixed state density matrix $\rho_{\mathcal{S}}$ can be decomposed into sums of density matrices that add up to the same resultant $\rho_{\mathcal{S}}$, but need not share the same eigenstates. For example, consider $\rho_{\mathcal{S}}^{a}$ and $\rho_{\mathcal{S}}^{b}$, representing two different preparations (i.e., involving measurement of two distinct, non-commuting observables) of two ensembles, each with multiple copies of a system $\mathcal{S}$. When they are randomly mixed in proportions $p^{a}$ and $p^{b}$, the resulting density matrix:

$$
\rho_{\mathcal{S}}^{a \vee b}=p^{a} \rho_{\mathcal{S}}^{a}+p^{b} \rho_{\mathcal{S}}^{b}
$$

is the complete description of the unified ensemble (see Schrödinger (1936); Jaynes (1957)).

Unless $\left[\rho_{\mathcal{S}}^{a}, \rho_{\mathcal{S}}^{b}\right]=0$, the eigenstates of $\rho_{\mathcal{S}}^{a \vee b}$ do not coincide with the eigenstates of the components. This feature makes it difficult to regard any density matrix in terms of probabilities and ignorance. Such ambiguity would be especially troubling if it arose in the description of an observer (or, for that matter, of any classical system). Ignorance interpretation - i.e., the idea that probabilities are observer's way of dealing with uncertainty about the outcome we have briefly explored in the discussion of the insider-outsider dichotomy, Eqs. (2.44), - requires at the very least that the set of events ("the sample space") exists independently of the information at hand - i.e, independently of $p^{a}$ and $p^{b}$ in the example above. Eigenstates of the density matrix do not supply such events, since the additional loss of information associated with mixing of the ensembles alters the candidate events.

Basis ambiguity would be disastrous for record states. Density matrices describing a joint state of the memory $\mathcal{A}$ and of the system $\mathcal{S}$ :

$$
\rho_{\mathcal{A S}}^{a \vee b}=p_{a} \rho_{\mathcal{A S}}^{a}+p_{b} \rho_{\mathcal{A S}}^{b}
$$

would have to be considered. In absence of einselection the eigenstates of such $\rho_{\mathcal{A S}}^{a \vee b}$ need not be even associated with a fixed set of record states of the presumably classical $\mathcal{A}$. Indeed, in general $\rho_{\mathcal{A S}}^{a \vee b}$ has a non-zero discord, ${ }^{6}$

\footnotetext{
${ }^{6}$ As we have seen in section IV, Eqs. (4.30) - (4.36), discord $\delta \mathcal{I}_{\mathcal{A}}(\mathcal{S} \mid \mathcal{A})=\mathcal{I}(\mathcal{S}: \mathcal{A})-\mathcal{J}_{\mathcal{A}}(\mathcal{S}: \mathcal{A})$ is a measure of
}

and its eigenstates are entangled (even when the above $\rho_{\mathcal{A S}}^{a \vee b}$ is separable, and can be expressed as mixture of matrices that have no entangled eigenstates). This would imply an ambiguity of what are the record states, precluding probability interpretation of measurement outcomes.

Observer may nevertheless have records of a system that is in an ambiguous situation described above. Thus;

$$
\rho_{\mathcal{A S}}^{a \vee b}=\sum_{k} w_{k}\left|A_{k}\right\rangle\left\langle A_{k}\right|\left(p_{k}^{a} \rho_{\mathcal{S}_{k}}^{a}+p_{k}^{b} \rho_{\mathcal{S}_{k}}^{b}\right)
$$

are admissible for an effectively classical $\mathcal{A}$ correlated with a quantum $\mathcal{S}$. Now the discord $\delta_{\mathcal{A}} \mathcal{I}(\mathcal{S} \mid \mathcal{A})=0$.

Mixing of ensembles of pairs of correlated systems one of which is subject to einselection does not lead to ambiguities discussed above. Discord $\delta_{\mathcal{A}}(\mathcal{S} \mid \mathcal{A})$ disappears in the einselected basis of $\mathcal{A}$, and the eigenvalues of the density matrices can behave as classical probabilities associated with 'events' - with the records. The "menu" of possible events in the sample space - e.g., records in memory - is fixed by einselection. Whether one can really justify this interpretation of the eigenvalues of the reducued density matrix is a separate question we are about to address.

\section{Probabilities from Envariance}

The view of 'the emergence of the classical' based on the environment - induced superselection has been occassionally described as "for practical purposes only" (see,

\footnotetext{
the "quantumness" of correlations. It should disappear as a result of the classical equivalence of two definitions of the mutual information, but is in general positive for quantum correlations, including, in particular, pre-decoherence $\rho_{\mathcal{S} \mathcal{A}}$. Discord is asymmetric, $\delta \mathcal{I}_{\mathcal{A}}(\mathcal{S} \mid \mathcal{A}) \neq \delta \mathcal{I}_{\mathcal{S}}(\mathcal{A} \mid \mathcal{S})$. Vanishing of $\delta \mathcal{I}_{\mathcal{A}}(\mathcal{S} \mid \mathcal{A})$ (i.e., of the discord in the direction exploring the classicality of the states of $\mathcal{A}$, on which $H\left(\rho_{\mathcal{S} \mid \mathcal{A}}\right)$ in the asymmetric $J_{\mathcal{A}}(S: A)$, Eq. (4.32), is conditioned) is necessary for the classicality of the measurement outcome (Zurek, 2000; Ollivier and Zurek, 2002, Zurek, 2002a). $\delta \mathcal{I}_{\mathcal{A}}(\mathcal{S} \mid \mathcal{A})$ can disappear as a result of decoherence in the einselected basis of the apparatus. Following einselection it is then possible to ascribe probabilities to the pointer states. In perfect measurements of Hermitean observables discord vanishes "both ways": $\delta \mathcal{I}_{\mathcal{A}}(\mathcal{S} \mid \mathcal{A})=\delta \mathcal{I}_{\mathcal{S}}(\mathcal{A} \mid \mathcal{S})=0$ for the pointer basis and for the eigenbasis of the measured observable correlated with it. Nevertheless, it is possible to encounter situations when vanishing of the discord in one direction is not accompanied by its vanishing "in reverse". Such correlations are "classical one way" (Zurek, 2002a) and could arise, for instance, in measurements of non-hermitean observables (which - as we have already noted - happen, in spite of the axiom (iii)).

This asymmetry between classical $\mathcal{A}$ and quantum $\mathcal{S}$ arises from the einselection. Classical record states are not arbitrary superpositions. Observer accesses his memory in the basis in which it is monitored by the environment. The information stored is effectively classical because it is being widely disseminated. States of the observers memory exist objectively - they can be found out through their imprints in the environment.
} 
e.g., Bell, 1990), to be contrasted with more fundamental (if nonexistent) solutions of the problem one could imagine (i.e., by modifying quantum theory; see Bell, 1987, 1990). This attitude can be in part traced to the reliance of einselection on reduced density matrices: For, even when explanations of all aspects of the effectively classical behavior are accepted in the framework of, say, Everett's MWI, and after the operational approach to objectivity and perception of unique outcomes based on the existential interpretation explained earlier is adopted, one major gap remains: Born's rule - axiom (v) connecting probabilities with the amplitudes, $p_{k}=\left|\psi_{k}\right|^{2}$ - has to be postulated in addition to axioms (o) - (ii). True, one can show that within the framework of einselection Born's rule emerges naturally (Zurek, 1998a). Decoherence is, however, based on on reduced density matrices. Now, since they were introduced by Landau (1927), it is known that "partial trace" leading to reduced density matrices is predicated on Born's rule (see Nielsen and Chuang, 2000, for discussion). Thus, derivations of Born's rule that employ reduced density matrices are open to charge of circularity (Zeh, 1997). Moreover, repeated attempts to justify $p_{k}=\left|\psi_{k}\right|^{2}$ within the no - collapse MWI (Everett, 1957 a\&b; DeWitt, 1970; DeWitt and Graham, 1973; Geroch, 1984) have failed (see e.g., Stein, 1984; Kent, 1990; Squires, 1990): The problem is their circularity: An appeal to the connection (especially in certain limiting procedures) between the smallnes of the amplitude and the vanishing of probabilities has to be made to establish that relative frequencies of events averaged over branches of the universal state vector are consitent with Born's rule. In particular, one must a claim that "maverick" branches of the MWI state vector that have "wrong" relative frequencies are of measure zero because their Hilbert space measures are small. This is circular, as noted even by the proponents (DeWitt, 1970).

My aim here is to look at the origin of ignorance, information, and, therefore, probabilities from a very quantum and fundamental perspective: Rather than focus on probabilities for an individual isolated system I shall - in the spirit of einselection, but without employing its usual tools such as trace or reduced density matrices - consider what the observer can (and cannot) know about a system entangled with its environment. Within this context I shall demonstrate that Born's rule follows from the very quantum fact that one can know precisely the state of the composite system and yet be provably ignorant of the state of its components. This is due to environment assited invariance or envariance, a hitherto unrecognised symmetry I am about to describe. Envariance of pure states is conspicuously missing from classical physics. It allows one to define ignorance as aconsequence of invariance, and thus to understand the origin of Born's rule, probabilities, and ultimately the origin of information through arguments based on assumptions different from Gleason's (1957) famous theorem. Rather, it is based on the Machian idea of 'relativity of quantum states' entertained by this author two decades ago (see p. 772 of Wheeler and Zurek, 1983), but not developed untill now. Envariance (Zurek, 2002b) addresses the question of meaning of these probabilities by defining "ignorance", and justifies a relative frequency argument, although in a manner different from the previous attempts.

\section{Envariance}

Environment - assisted invariance is a symmetry exhibited by the system $\mathcal{S}$ correlated with the other system (usually 'the environment' $\mathcal{E}$ ). When a state of the composite $\mathcal{S} \mathcal{E}$ can be transformed by $u_{\mathcal{S}}$ acting solely on the Hilbert space $\mathcal{H}_{\mathcal{S}}$, but the effect of this transformation can be undone with an appropriate $u_{\mathcal{E}}$ acting only on $\mathcal{H}_{\mathcal{E}}$, so that the joint state $\left|\psi_{\mathcal{S E}}\right\rangle$ remains unaltered;

$$
u_{\mathcal{S}} u_{\mathcal{E}}\left|\psi_{\mathcal{S E}}\right\rangle=\left|\psi_{\mathcal{S E}}\right\rangle
$$

such $\left|\psi_{\mathcal{S E}}\right\rangle$ is envariant under $u_{\mathcal{S}}$. Generalization to mixed $\rho_{\mathcal{S E}}$ is obvious, but we shall find it easier to assume that $\mathcal{S E}$ has been purified in the usual fashion i.e., by enlarging the environment.

Envariance is best elucidated by considering an example - an entangled state of $\mathcal{S}$ and $\mathcal{E}$. It can be expressed in the Schmidt basis as:

$$
\left|\psi_{\mathcal{S E}}\right\rangle=\sum_{k} \alpha_{k}\left|s_{k}\right\rangle\left|\varepsilon_{k}\right\rangle,
$$

where $\alpha_{k}$ are complex, while $\left\{\left|s_{k}\right\rangle\right\}$ and $\left\{\left|\varepsilon_{k}\right\rangle\right\}$ are orthonormal. For $\left|\psi_{\mathcal{S E}}\right\rangle$ (and, hence - given our above remark about purification - for any system correlated with the environment) it is easy to demonstrate:

Lemma 6.1: Unitary transformations co-diagonal with the Schmidt basis of $\left|\psi_{\mathcal{S E}}\right\rangle$ leave it envariant.

The proof relies on the form of such transformations:

$$
u_{\mathcal{S}}^{\left\{\left|s_{k}\right\rangle\right\}}=\sum_{k} e^{i \sigma_{k}}\left|s_{k}\right\rangle\left\langle s_{k}\right|
$$

where $\sigma_{k}$ is a phase. Hence;

$$
u_{\mathcal{S}}^{\left\{\left|s_{k}\right\rangle\right\}}\left|\psi_{\mathcal{S E}}\right\rangle=\sum_{k} \alpha_{k} e^{i \sigma_{k}}\left|s_{k}\right\rangle\left|\varepsilon_{k}\right\rangle,
$$

can be undone by:

$$
u_{\mathcal{E}}^{\left\{\left|\varepsilon_{k}\right\rangle\right\}}=\sum_{k} e^{i \epsilon_{k}}\left|\varepsilon_{k}\right\rangle\left\langle\varepsilon_{k}\right|
$$

providing that $\epsilon_{k}=2 \pi l_{k}-\sigma_{k}$ for some integer $l_{k}$. QED.

Thus, phases associated with the Schmidt basis are envariant. We shall see below that they are the only envariant property of entangled states. The transformations defined by Eq. (6.8) are rather specific - they share (Schmidt) eigenstates. Still, their existence leads us to:

Theorem 6.1: Local description of the system $\mathcal{S}$ entangled with a causally disconnected environment $\mathcal{E}$ must 
not depend on the phases of the coefficients $\alpha_{k}$ in the Schmidt decomposition of $\left|\psi_{\mathcal{S E}}\right\rangle$.

It follows that all the measurable properties of the $\mathcal{S}$ are completely specified by the list of pairs $\left\{\left|\alpha_{k}\right| ;\left|s_{k}\right\rangle\right\}$. An equivalent way of establishing this phase envariance theorem appeals even more directly to causality: Phases of $\left|\psi_{\mathcal{S E}}\right\rangle$ can be arbitrarily changed by acting on $\mathcal{E}$ alone (e.g, by the local Hamiltonian with eigenstates $\left|\varepsilon_{k}\right\rangle$, generating evolution of the from of Eq. (6.9)). But causality prevents faster than light communication. Hence, no measurable property of $\mathcal{S}$ can be effected. QED.

Phase envariance theorem will turn out to be the crux of our argument. It relies on an input - entanglement and causality - which has not been employed to date in discussions of the origin of probabilities. In particular, this input is different and more "physical" than that of the succesfull derivation of Born's rules by Gleason (1957).

We also note that information contained in the "data base" $\left\{\left|\alpha_{k}\right| ;\left|s_{k}\right\rangle\right\}$ implied by the Theorem 6.1 is the same as in the reduced density matrix of the system $\rho_{\mathcal{S}}$ : Although we do not yet know probabilities of various $\left|s_{k}\right\rangle$, preferred basis of $\mathcal{S}$ has been singled out Schmidt states (sometimes regarded as instantaneous pointer states; see e.g. Albrecht, $(1992 ; 1993)$ ) play a special role as the eigenstates of envariant transformations. Moreover, probabilities can depend on $\left|\alpha_{k}\right|$ (but not on the phases). We still do not know that $p_{k}=\left|\alpha_{k}\right|^{2}$.

The causality argument we could have used to establis Theorem 6.1 applies of course to arbitrary transformations one could perform on $\mathcal{E}$. However, such transformations would not be in general envariant (i.e., could not be undone by acting on $\mathcal{S}$ alone). Indeed - by the same token - all envariant transformations must be diagonal in Schmidt basis:

Lemma 6.2: All of the unitary envariant transformations of $\left|\psi_{\mathcal{S E}}\right\rangle$ have Schmidt eigenstates.

The proof relies on the fact that other unitary transformations would rotate Schmidt basis, $\left|s_{k}\right\rangle \rightarrow\left|\tilde{s}_{k}\right\rangle$. The rotated basis becomes a new 'Schmidt', and this fact cannot be affected by unitary transformations of $\mathcal{E}$ - by state rotations in the environment. But a state that has a different Schmidt decomposition from the original $\left|\psi_{\mathcal{S E}}\right\rangle$ is different. Hence, unitary transformation must be codiagonal with Schmidt states to leave it envariant. QED.

\section{Born's rule from envariance}

When absolute values of some of the coefficients in Eq. (6.7) are equal, any orthonormal basis is 'Schmidt' in the corresponding subspace of $\mathcal{H}_{\mathcal{S}}$. This implies envariance of more general nature, e.g. under a swap;

$$
u_{\mathcal{S}}(k \leftrightarrow j)=e^{i \phi_{k j}}\left|s_{k}\right\rangle\left\langle s_{j}\right|+\text { h.c. }
$$

Swap can be generated by a phase rotation, Eq. (6.8), but in a basis complementary to the one swapped. Its envariance does not contradict Lemma 6.2, as any orthonormal basis in this case is also 'Schmidt'). So, when $\left|\alpha_{k}\right|=\left|\alpha_{j}\right|$, the effect of a swap on the system can be undone by an obvious counterswap in the environment:

$$
u_{\mathcal{S}}(k \leftrightarrow j)=e^{-i\left(\phi_{k j}+\phi_{k}-\phi_{j}+2 \pi l_{k j}\right)}\left|\varepsilon_{k}\right\rangle\left\langle\varepsilon_{j}\right|+\text { h.c. }
$$

Swap can be applied to states that do not have equal absolute values of the coefficients, but in that case it is no longer envariant. Partial swaps can be also generated e.g., by underrotating or by a $u_{\mathcal{S}}^{\left\{\left|r_{i}\right\rangle\right\}}$, Eq. (6.8), but with the eigenstates $\left\{\left|r_{i}\right\rangle\right\}$ intermediate between these of the swapped and the complementary (Hadamard) basis. Swap followed by a counterswap exchanges coefficients of the swapped states in the Schmidt expansion, Eq. (6.7).

Classical correlated states can also exhibit something akin to envariance under a classical version of "swaps". For instance, a correlated state of system and an apparatus described by: $\rho_{\mathcal{S A}} \sim\left|s_{k}\right\rangle\left\langle s_{k}|| A_{k}\right\rangle\left\langle A_{k}\right|+$ $\left|s_{j}\right\rangle\left\langle s_{j}|| A_{j}\right\rangle\left\langle A_{j}\right|$ can be swapped and counterswapped. The corresponding transformations would be still given by, in effect, Eqs. (6.11) - (6.12), but without phases, and swaps could no longer be generated by the rotations around the complementary basis. This situation corresponds to the "outsiders view" of the measurement process, Eq. (2.44c): Outsider can be aware of the correlation between the system and of the apparatus, but ignorant of their individual states. This connection between ignorance and envariance shall be exploited below.

Envariance based on ignorance may be found in the classical setting, but envariance of pure states is purely quantum: Observers can know perfectly the quantum joint state of $\mathcal{S E}$, yet be provably ignorant of $\mathcal{S}$. Consider a measurement carried out on the state vector of $\mathcal{S E}$ from the point of view of envariance:

$$
\left|A_{0}\right\rangle \sum_{k=1}^{N}\left|s_{k}\right\rangle\left|\varepsilon_{k}\right\rangle \rightarrow \sum_{k=1}^{N}\left|A_{k}\right\rangle\left|s_{k}\right\rangle\left|\varepsilon_{k}\right\rangle \sim\left|\Phi_{\mathcal{S} \mathcal{A} \mathcal{E}}\right\rangle
$$

Above, we have assumed that the absolute values of the coefficients are equal (and omitted them for notational simplicity). We also have ignored phases (which need not be equal) since - by the phase envariance theorem - they will not influence the state (and, hence, the probabilities) associated with $\mathcal{S}$.

Before the measurement observer with access to $\mathcal{S}$ cannot notice swaps in the state (such as Eq. (6.13)) with equal absolute values of the Schmidt coefficients. This follows from envariance of the pre-measurement $\left|\psi_{\mathcal{S E}}\right\rangle$ under swaps, Eq. (6.11).

One could argue this point in more detail by comparing what happens for two very different input states; an entangled $\left|\psi_{\mathcal{S E}}\right\rangle$ with equal absolute values of Schmidt coefficients and a product state:

$$
\left|\varphi_{\mathcal{S E}}\right\rangle=\left|s_{J}\right\rangle\left|\varepsilon_{J}\right\rangle
$$

When observer knows he is dealing with $\varphi_{\mathcal{S E}}$, he knows the state and can predict outcome of the corresonding measurement on $\mathcal{S}$ : Schrödinger equation or just the resulting truth table, Eq. (6.5), imply with certainty that 
his state - the future state of his memory - will be $\left|A_{J}\right\rangle$. Moreover, swaps involving $\left|s_{J}\right\rangle$ are not envariant for $\varphi_{\mathcal{S E}}$. They just swap the outcomes (i.e. when $u_{\mathcal{S}}(J \leftrightarrow L)$ precedes the measurement, memory will end up in $\left|A_{L}\right\rangle$ ).

By contrast,

$$
\left|\psi_{\mathcal{S E}}\right\rangle \sim \sum_{k=1}^{N} e^{i \phi_{k}}\left|s_{k}\right\rangle\left|\varepsilon_{k}\right\rangle
$$

is envariant under swaps. This allows the observer (who knows the joint state of $\mathcal{S E}$ exactly) to conclude that the probabilities of all the envariantly swappable outcomes must be the same. Observer cannot predict his memory state after the measurement of $\mathcal{S}$ because he knows too much; the exact combined state of $\mathcal{S E}$.

For completeness, we note that when there are system states that are absent from the above sum - i.e., states that 'appear with zero amplitude' - they cannot be envariantly swapped with the states present in the sum. Of course, observer can predict with certainty he will not detect any of the corresponding zero - amplitude outcomes.

This argument about the ignorance of the observer concerning his future state - concerning the outcome of the measurement he is about to perform - is based on his perfect knowledge of a joint state of $\mathcal{S E}$.

Probabilities refer to the guess observer makes on the basis of his information before the measurement about the state of his memory - "the future outcome" - after the measurement. As the left hand side of Eq. (6.13) is envariant under swaps of the system states, probabilities of all the states must be equal. Thus, by normalisation;

$$
p_{k}=1 / N \text {. }
$$

Moreover, probability of $n$ mutually exclusive events that all appear in Eq. (6.13) with equal coefficients must be:

$$
p_{k_{1} \vee k_{2} \vee \ldots \vee k_{n}}=n / N .
$$

This concludes discussion of the equal probability case. Our case rests on the independence of the state of $\mathcal{S}$ entangled with $\mathcal{E}$ from the phases of the coefficients in the Schmidt representation - the Theorem 6.1 - which in the case of equal coefficients Eq. (6.13), allows envariant swapping, and yields Eqs. (6.14)-(6.15).

After a measurement situation changes. In accord with our preceding discussion we interpret presence of the term $\left|A_{k}\right\rangle$ in Eq. (6.13) as evidence that an outcome $\left|s_{k}\right\rangle$ can be (or indeed has been - the language here is somewhat dependent on the interpretation) recorded. Conversely, absence of some $\left|A_{k^{\prime}}\right\rangle$ in the sum above implies that the outcome $\left|s_{k^{\prime}}\right\rangle$ cannot occur. After a measurement memory of the observer who has detected $\left|s_{k}\right\rangle$ will contain the record $\left|A_{k}\right\rangle$. Futher measurements of the same observable on the same system will confirm that $\mathcal{S}$ is in indeed in the state $\left|s_{k}\right\rangle$.

This post-measurement state is still envariant, but only under swaps that involve jointly the state of the system and the correlated state of the memory:

$$
u_{\mathcal{A S}}(k \leftrightarrow j)=e^{i \phi_{k j}}\left|s_{k}, A_{k}\right\rangle\left\langle s_{j}, A_{j}\right|+\text { h.c. }
$$

Thus, if another observer ('Wigner's friend') was getting ready to find out - either by direct measurement of $\mathcal{S}$ or by communicating with observer $\mathcal{A}$ - the outcome of his measurement, he would be (on the basis of envariance) provably ignorant of the outcome $\mathcal{A}$ has detected, but could be certain of the $\mathcal{A S}$ correlation. We shall employ this joint envariance in the discussion of the case of unequal probabilities immediately below.

Note that our reasoning does not really appeal to the "information lost in the environment" in a sense in which this phrase is often used. Perfect knowledge of the combined state of the system and the environment is the basis of the argument for the ignorance od $\mathcal{S}$ alone: For entangled $\mathcal{S E}$, perfect knowlegde of $\mathcal{S E}$ is incompatible with perfect knowledge of $\mathcal{S}$. This is really a consequence of indeterminacy - joint observables with entangled eigenstates such as $\psi_{\mathcal{S E}}$ simply do not commute (as the reader is invited to verify) with the observables of the system alone. Hence, ignorance associated with envariance is ultimately mandated by Heisenberg indeterminacy.

The case of unequal coefficients can be reduced to the case of equal coefficients. This can be done in several ways, of which we choose one that makes use of the preceding discussion of envariance of the post-measurement state. We start with:

$$
\left|\Phi_{\mathcal{S A E}}\right\rangle \sim \sum_{k=1}^{N} \alpha_{k}\left|A_{k}\right\rangle\left|s_{k}\right\rangle\left|\varepsilon_{k}\right\rangle
$$

where $\alpha_{k} \sim \sqrt{m}_{k}$ and $m_{k}$ is a natural number (and, by phase envariance theorem, we drop the phases). To get an envariant state we "increase the resolution" of $\mathcal{A}$ by assuming that;

$$
\left|A_{k}\right\rangle=\sum_{j_{k}=1}^{m_{k}}\left|a_{j_{k}}\right\rangle / \sqrt{m}_{k}
$$

An increase of resolution is a standard trick, used in classical probability theory "to even the odds". Note that we assume that basis states such as $\left|A_{k}\right\rangle$ are normalised (as they must be in a Hilbert space). This leads to:

$$
\left|\Phi_{\mathcal{S A E}}\right\rangle \sim \sum_{k=1}^{N} \sqrt{m}_{k} \frac{\sum_{j_{k}=1}^{m_{k}}\left|a_{j_{k}}\right\rangle}{\sqrt{m}_{k}}\left|s_{k}\right\rangle\left|\varepsilon_{k}\right\rangle
$$

We now assume that $\mathcal{A}$ and $\mathcal{E}$ interact (e.g., through a c-shift of section 2 , with a truth table $\left|a_{j_{k}}\right\rangle\left|\varepsilon_{k}\right\rangle \rightarrow$ $\left|a_{j_{k}}\right\rangle\left|e_{j_{k}}\right\rangle$ where $\left\{\left|e_{j_{k}}\right\rangle\right\}$ are all orthonormal), so that after simplifying and re-arranging terms we get a sum, over a new 'fine-grained' index, with the states of $\mathcal{S}$ that remain the same within 'coarse - grained cells', i.e., intervals measured by $m_{k}$ :

$$
\left|\tilde{\Phi}_{\mathcal{S A E}}\right\rangle \sim \sum_{k=1}^{N}\left|s_{k}\right\rangle\left(\sum_{j_{k}=1}^{m_{k}}\left|a_{j_{k}}\right\rangle\left|e_{j_{k}}\right\rangle\right)=\sum_{j=1}^{M}\left|s_{k(j)}\right\rangle\left|a_{j}\right\rangle\left|e_{j}\right\rangle
$$


Above, $M=\sum_{k=1}^{N} m_{k}$, and $k(j)=1$ for $j \leq m_{1}, k(j)=$ 2 for $j \leq m_{1}+m_{2}$, etc. The above state is envariant under combined swaps;

$$
u_{\mathcal{S A}}\left(j \leftrightarrow j^{\prime}\right)=\exp \left(i \phi_{j j^{\prime}}\right)\left|s_{k(j)}, a_{j}\right\rangle\left\langle a_{j^{\prime}}, s_{k\left(j^{\prime}\right)}\right|+h . c .
$$

Suppose that an additional observer measures $\mathcal{S} \mathcal{A}$ in the obviously swappable joint basis. By our equal coefficients argument, Eq. (6.14), we get $p\left(s_{k(j)}, a_{j}\right)=1 / M$. But the observer can ignore states $a_{j}$. Then the probability of different Schmidt states of $\mathcal{S}$ is, by Eq. (6.15);

$$
p\left(s_{k}\right)=m_{k} / M=\left|\alpha_{k}\right|^{2} \text {. }
$$

This is Born's rule.

The case with coefficients that do not lead to commensurate probabilities can be treated by assuming continuity of probabilities as a function of the amplitudes and taking appropriate (and obvious) limits. This can be physically motivated: One would not expect probabilities to change drastically depending on the infinitesimal changes of the state. One can also extend the strategy outlined above to deal with probabilities (and probability densities) in cases such as $|s(x)\rangle$, i.e. when the index of the state vector changes continuously. This can be accomplished by discretising it (so that the measurement of Eq. (6.17) correlates different apparatus states with small intervals of $x$ ) and then repeating the strategy of Eqs. (6.17) - (6.21). The wavefuction $s(x)$ should be sufficiently smooth for this strategy to succeed.

We note that the "increase of resolution" we have exploited, Eqs. (6.18)-(6.21), need not be physically implemented for the argument to go through: The very possibility of carrying out these steps within the quantum formalism forces one to adopt Born's rule. For example, if the apparatus did not have the requisite extra resolution, Eq. (6.18), interaction of the environment with a still different 'counterweight' system $\mathcal{C}$ that yields

$$
\left|\Psi_{\mathcal{S A E C}}\right\rangle=\sum_{k=1}^{N} \sqrt{m}_{k}\left|s_{k}\right\rangle\left|A_{k}\right\rangle\left|\varepsilon_{k}\right\rangle\left|C_{k}\right\rangle
$$

would lead one to the Born's rule through steps similar to these we have invoked before, providing that $\left|C_{k}\right\rangle$ \} has the requisite resolution, $\left|C_{k}\right\rangle=\sum_{j_{k}=1}^{m_{k}}\left|c_{j_{k}}\right\rangle /{\sqrt{m_{k}}}_{k}$. Interaction resulting in a correlation, Eq. (6.22), can occur between $\mathcal{E}$ and $\mathcal{C}$, and happen far from the system of interest or from the apparatus. Thus, it will not influence probabilities of the outcomes of measurements carried out on $\mathcal{S}$ or of the records made by $\mathcal{A}$. Yet, the fact that it can happen leads us to the desired conclusion.

\section{Relative frequencies from envariance}

Relative frequency is a common theme studied with the aim of elucidating the physical meaning of probabilities in quantum theory (Everett, 1957; Hartle, 1968; DeWitt, 1970; Graham, 1970; Farhi, Goldstone, and Gutmann,
1989; Aharonov and Resnik, 2002). In particular, in the context of the "no collapse" MWI relative frequency seem to offer the best hope of arriving at the Born's rule and elucidating its physical significance. Yet, it is generally acknowledged that the MWI derivations offered to date have failed to attain this goal (Kent, 1990).

We postpone brief discussion of these efforts to the next section, and describe an approach to relative frequencies based on envariance. Consider an ensemble of many $(\mathcal{N})$ distinguishable systems prepared in the same initial state:

$$
\left|\sigma_{\mathcal{S}}\right\rangle=\alpha|0\rangle+\beta|1\rangle
$$

We focus on the two state case to simplify the notation. We also assume that $|\alpha|^{2}$ and $|\beta|^{2}$ are commensurate, so that the state vector of the whole ensemble of correlated triplets $\mathcal{S} \mathcal{A} \mathcal{E}$ after the requisite increases of resolution (see Eqs. (6.18)-(6.20) above) is given by:

$$
\left|\Phi_{\mathcal{S} \mathcal{A} \mathcal{E}}^{\mathcal{N}}\right\rangle \sim\left(\sum_{j=1}^{m}|0\rangle\left|a_{j}\right\rangle\left|e_{j}\right\rangle+\sum_{j=m+1}^{M}|1\rangle\left|a_{j}\right\rangle\left|e_{j}\right\rangle\right)^{\otimes \mathcal{N}}
$$

save for the obvious normalisation. This state is envariant under swaps of the joint states $\left|s, a_{j}\right\rangle$, as they appear with the same (absolute value) of the amplitude in Eq. (6.24). (By Theorem 6.1 we can omitt phases.)

After the exponentiation is carried out, and the resulting product states are sorted by the number of 0's and 1 's in the records, we can calculate the number of terms with exactly $n 0$ 's, $\nu_{\mathcal{N}}(n)=\left(\begin{array}{c}\mathcal{N} \\ n\end{array}\right) m^{n}(M-m)^{\mathcal{N}-n}$. To get probability, we normalise:

$p_{\mathcal{N}}(n)=\left(\begin{array}{c}\mathcal{N} \\ n\end{array}\right) \frac{m^{n}(M-m)^{\mathcal{N}-n}}{M^{\mathcal{N}}}=\left(\begin{array}{c}\mathcal{N} \\ n\end{array}\right)|\alpha|^{2 n}|\beta|^{2(\mathcal{N}-n)}$.

This is the distribution one would expect from Born's rule. To establish the connection with relative frequencies we appeal to the de Moivre - Laplace theorem (Gnedenko, 1982) which allows one to approximate above $p_{\mathcal{N}}(n)$ with a Gaussian:

$$
p_{\mathcal{N}}(n) \simeq \frac{1}{\sqrt{2 \pi \mathcal{N}}|\alpha \beta|} e^{-\frac{1}{2}\left(\frac{n-\mathcal{N}|\alpha|^{2}}{\sqrt{\mathcal{N}|\alpha \beta|}}\right)^{2}}
$$

This last step requires large $\mathcal{N}$, but our previous discussion including Eq. (6.25) is valid for arbitrary $\mathcal{N}$. Indeed, equation (6.21) can be regarded as the $\mathcal{N}=1$ case.

Nevertheless, for large $\mathcal{N}$ relative frequency is sharply peaked around the expected $\langle n\rangle=\mathcal{N}|\alpha|^{2}$. Indeed, in the limit $\mathcal{N} \rightarrow \infty$ appropriately rescaled $p_{\mathcal{N}}(n)$ tends to a Dirac $\delta\left(v-|\alpha|^{2}\right)$ in relative frequency $v=n / \mathcal{N}$. This justifies the relative frequency interpretation of the squares of amplitudes as probabilities in the MWI context. 'Maverick universes' with different relative frequencies exist, but have a vanishing probability (and not just vanishing Hilbert space measure) for large $\mathcal{N}$.

Our derivation of the physical significance of probabilities - while it led to the relative frequency argument 
- was based on a very different set of assumptions than previous derivations. The key idea behind it is the connection between a symmetry (envariance) and ignorance (impossibility of knowing something). The unusual feature of our argument is that this ignorance (for an individual system $\mathcal{S}$ ) is demonstrated by appealing to the perfect knowledge of the larger joint system that includes $\mathcal{S}$ as a subsystem.

We emphasize that one could not carry out the basic step of our argument - the proof of the independence of the likelihoods from the phases of the Schmidt expansion coefficients - for an equal amplitude pure state of a single, isolated system. The problem with: $|\psi\rangle=N^{-\frac{1}{2}} \sum_{k}^{N} \exp \left(i \phi_{k}\right)|k\rangle$ is the accessibility of the phases. Consider, for instance; $|\psi\rangle \sim|0\rangle+|1\rangle-|2\rangle$ and $\left|\psi^{\prime}\right\rangle \sim|2\rangle+|1\rangle-|0\rangle$. In absence of decoherence swapping of $k$ 's is detectable: Interference measurements (i.e., measurements of the observables with phase-dependent eigenstates $|1\rangle+|2\rangle ;|1\rangle-|2\rangle$, etc.) would have revealed the difference between $|\psi\rangle$ and $\left|\psi^{\prime}\right\rangle$. Indeed, given an ensemble of identical pure states an observer will simply find out what they are. Loss of phase coherence is essential to allow for the shuffling of the states and coefficients.

Note that in our derivation environment and einselection play an additional, more subtle role: Once a measurement has taken place - i.e., a correlation with the apparatus or with the memory of the observer was established - one would hope that records will retain validity over a long time, well beyond the decoherence timescale. This is a pre-condition for the axiom (iv). Thus, a "collapse" from a multitude of possibilities to a single reality can be confirmed by subsequent measurements only in the einselected pointer basis.

\section{Other approaches to probabilities}

Gnedenko (1982), in his classic textbook, lists three classical approaches to probability:

a. Definitions that appeal to relative frequency of occurrence of events in a large number of trials.

b. Definitions of probability as a measure of certainty of the observer.

c. Definitions that reduce probability to the more primitive notion of equal likelihood.

In the quantum setting, the relative frequency approach has been to date the most popular, especially in the context of the "no collapse" MWI" (Everett, 1957a\&b; Graham, 1970; DeWitt, 1970). Counting the number of the "clicks" seems most directly tied to the experimental manifestations of probability. Yet, Everett interpretation versions were generally found lacking (Kent, 1990; Squires, 1990), as they relied on circular reasoning, invoking without physical justification an abstract measure of Hilbert space to obtain a physical measure (frequency). Some of the criticisms seem relevant also for the versions of this approach that allow for the measurement postulates (iii) and (iv) (Hartle, 1968; Farhi, Goldstone and Guttmann, 1989). Nevertheless, for the infinite ensembles considered in the above references, (where, in effect, the Hilbert space measure of the MWI branches that violate relative frequency predictions is zero) that the eigenvalues of the frequency operator acting on a large or infinite ensemble of identical states will be consistent with the (Born formula) prescription for probabilities.

However, the infinite size of the ensemble necessary to prove this point is troubling (and unphysical) and taking the limit starting from a finite case is difficult to justify (Stein, 1984; Kent, 1990; Squires, 1990). Moreover, the frequency operator is a collective observable of the whole ensemble. It may be possible to relate observables defined for such an infinite ensemble supersystem to the states of individual subsystems, but the frequency operator does not do it. This is well illustrated by the gedankenexperiment envisaged by Farhi et al. (1989). To provide a physical implementation of the frequency operator they consider a version of the Stern-Gerlach experiment where all the spins are attached to a common lattice, so that - during the passage through the inhomogeneity of the magnetic field - the center of mass of the whole lattice is deflected by an angle proportional to the projection of the net magnetic moment associated with the spins on the direction defined by the field gradient. The deflection is proportional to the eigenvalue of the frequency operator that is then a collective observable states of individual spins remain in superpositions, uncorrelated with anything outside. This difficulty can be addressed with the help of decoherence (Zurek, 1998a), but using decoherence without justifying Born's formula first is fraught with danger of circularity.

Measure of certainty seems to be a rather vague concept. Yet, Cox (1946) has demonstrated that Boolean logic leads - after addition of a few reasonable assumptions - to the definition of probabilities that, in a sense, appear as an extension of the logical truth values. However, the rules of symbolic logic that underlie Cox's theorems are classical. One can adopt this approach (Zurek, 1998a) to probabilities in quantum physics only after decoherence "intervenes" restoring the validity of the distributive law, which is not valid in quantum physics (Birkhoff and von Neumann, 1936).

One can carry out equal likelihood approach in the context of decoherence (Zurek, 1998a). The problem is - as pointed out before - the use of trace, and the dangers of circularity. An attempt to pursue a strategy akin to equal likelihood in the quantum setting at the level of pure states of individual systems has been also made by Deutsch in his (unpublished) "signalling" approach to probabilities. The key idea is to consider a source of pure states, and to find out when the permutations of a set of basis states can be detected, and, therefore, used for communication. When permutations are undetectable, probabilities of the permuted set of states are declared equal. The problem with this idea (or with its more formal ver- 
sion described by DeWitt, 1998) is that it works only for superposition that have all the coefficients identical, including their phases. Thus, as we have already noted, for closed systems phases matter and there is no invariance under swapping. In a recent paper Deutsch (1999) has adopted a different approach based on decision theory. The basic argument focuses again on individual states of quantum systems, but - as noted in the critical comment by Barnum et al. (2000) - seems to make appeal to some of the aspects of decision theory that do depend on probabilities. In my view, it also leaves the problem of the phase dependence of the coefficients unaddressed.

Among other approaches, recent work of Gottfried (2000) shows that in a discrete quantum system coupled with a continuous quantum system Born's formula follows from the demand that the continuous system should follow classical mechanics in the appropriate limit. A somewhat different strategy, with a focus on the coincidences of the expected magnitude of fluctuations was proposed by Aharonov and Resnik (2002).

In comparison with all of the above strategies, 'probabilities from envariance' is the most radically quantum, in that it ultimately relies on entanglement (which is still sometimes regarded as 'a paradox', and 'to be explained': I have used it as an explanation). This may be the reason why it has not been discovered untill now. The insight offered by envariance into the nature ignorance and information sheds a new light on probabilities in physics. The (very quantum) ability to prove ignorance of a part of a system by appealing to perfect knowledge of the whole may resolve some of the difficulties of the classical approaches.

\section{ENVIRONMENT AS A WITNESS}

Emergence of classicality can be viewed either as a consequence of the widespread dissemination of the information about the pointer states through the environment, or as a result of the censorship imposed by decoherence. So far I have focused on this second view, defining $e x$ istence as persistence - predictability in spite of the environmental monitoring. Predictability sieve is a way of discovering states that are classical in this sense (Zurek, 1993a\&b; Zurek, Habib and Paz, 1993; Gallis, 1996).

A complementary approach focusses not on the system, but on the records of its state spread throughout the environment. Instead of "the least perturbed states" one can ask "what states of the system are easiest to discover by looking at the environment". Thus, environment is no longer just a source of decoherence, but acquires a role of a communication channel with a basis - dependent noise that is minimised by the preferred pointer states.

This approach can be motivated by the old dilemma: On the one hand, quantum states of isolated systems are purely "epistemic" (see e.g., Peres, (1993); Fuchs and Peres (2000)). Quantum cryptography (Bennett and DiVincenzo, 2000; Nielsen and Chuang, 2000, and refer- ences therein) uses this impossibility of finding out what is an unknown state of an isolated quantum system. On the other hand classical reality seems to be made up of quantum building blocks: States of macroscopic systems exist objectively - they can be found out by many observers independently without being destroyed or reprepared. So - the question arises - how can objective existence - the "reality" of the classical states - emerge from "purely epistemic" wavefunctions?

There is not much one can do about this in case of a single state of an isolated quantum system. But open systems are subject to einselection and can bridge the chasm dividing their epistemic and ontic roles. The most direct way to see this arises from the recognition of the fact that we never directly observe any system. Rather, we discover states of macroscopic systems from the imprints they make on the environment: A small fraction of the photon environment intercepted by our eyes is often all that is needed. States that are recorded most redundantly in the rest of the Universe (Zurek, 1983; 1998a; 2000) are also easiest to discover. They can be found out indirectly, from multiple copies of the evidence imprinted in the environment, without a threat to their existence. Such states exist and are real - they can be found out without being destroyed as if they were really classical.

Environmental monitoring creates an ensemble of "witness states" in the subsystems of the environment, that allow one to invoke some of the methods of the statistical interpretation (Ballentine, 1970) while subverting its ideology - to work with an ensemble of objective evidence of a state of a single system. From this ensemble of witness states one can infer the state of the quantum system that has led to such "advertising". This can be done without disrupting the einselected states.

Predictability sieve selects states that entangle least with the environment. Question about predictability simultaneously lead to states that are most redundantly recorded in the environment. Indeed, this idea touches on the "quantum Darwinism" we have alluded to in the introduction: The einselected pointer states are not only best in surviving the environment, but, also, they broadcast the information about themselves - spread out their "copies" - throughout the rest of the Universe: Amplified information is easiest to amplify. This leads to analogies with "fitness" in the Darwinian sense, and suggests looking at einselection as a sort of natural selection.

\section{A. Quantum Darwinism}

Consider the "bit by byte" example of Section IV. Spin - system $\mathcal{S}$ is correlated with the environment:

$$
\begin{aligned}
\left|\psi_{\mathcal{S E}}\right\rangle & =a|\uparrow\rangle|00 \ldots 0\rangle+b|\downarrow\rangle|11 \ldots 1\rangle \\
& =a|\uparrow\rangle\left|\mathcal{E}_{\uparrow}\right\rangle+b|\downarrow\rangle\left|\mathcal{E}_{\downarrow}\right\rangle
\end{aligned}
$$

The basis $\{|\uparrow\rangle,|\downarrow\rangle\}$ of $\mathcal{S}$ is singled out by the redundancy of the record. By comparison, the same $\left|\psi_{\mathcal{S E}}\right\rangle$ is:

$$
\left|\psi_{\mathcal{S E}}\right\rangle=|\odot\rangle(a|00 \ldots 0\rangle+b|11 \ldots 1\rangle) \sqrt{2}
$$




$$
\begin{aligned}
& +|\otimes\rangle(a|00 \ldots 0\rangle-b|11 \ldots 1\rangle) / \sqrt{2} \\
& =\left(|\odot\rangle\left|\mathcal{E}_{\odot}\right\rangle+|\otimes\rangle\left|\mathcal{E}_{\otimes}\right\rangle\right) / \sqrt{2}
\end{aligned}
$$

in terms of the Hadamard-transformed $\{|\odot\rangle,|\otimes\rangle\}$.

One can find out whether $\mathcal{S}$ is $|\uparrow\rangle$ or $|\downarrow\rangle$ from a small subset of the environment bits. By contrast, states $\{|\odot\rangle,|\otimes\rangle\}$ cannot be easily inferred from the environment. States $\left\{\left|\mathcal{E}_{\odot}\right\rangle,\left|\mathcal{E}_{\otimes}\right\rangle\right\}$ are typically not even orthogonal, $\left\langle\mathcal{E}_{\odot} \mid \mathcal{E}_{\otimes}\right\rangle=|a|^{2}-|b|^{2}$. And even when $|a|^{2}-|b|^{2}=0$, the record in the environment is fragile: Only one relative phase distinguishes $\left|\mathcal{E}_{\odot}\right\rangle$ from $\left|\mathcal{E}_{\otimes}\right\rangle$ in that case, in contrast with multiple records of the pointer states in $\left|\mathcal{E}_{\uparrow}\right\rangle$ and $\left|\mathcal{E}_{\downarrow}\right\rangle$. Remarks that elaborate this observation follow. They correspond to several distinct measures of 'fitness' of states.

\section{Consensus and algorithmic simplicity}

¿From the state vector $\left|\psi_{\mathcal{S E}}\right\rangle$, Eqs. (7.1) and (7.2), observer can find the state of the quantum system just by looking at the environment. To accomplish this, the total $N$ of the environment bits can be divided into samples of $n$ bits each, with $1 \ll n \ll N$. These samples can be then measured using observables that are the same within each sample, but differ between samples. They may correspond, for example, to different antipodal points in the Bloch spheres of the environment bits. In the basis $\{|0\rangle,|1\rangle\}$ (or bases closely aligned with it) the record inferred from the bits of information scattered in the environment will be easiest to come by. Thus, starting from the environment part of $\left|\psi_{\mathcal{S E}}\right\rangle$, Eq. (7.1), the observer can find out, with no prior knowledge, the state of the system: Redundancy of the record in the environment allows for a trial-and-error 'indirect' approach while leaving the system untouched.

In particular, measurement of $n$ environment bits in a Hadamard transform of the basis $\{|0\rangle,|1\rangle\}$ yields a random-looking sequence of outcomes (i.e., $\left.\left\{|+\rangle_{1},|-\rangle_{2}, \ldots|-\rangle_{n}\right\}\right)$. This record is algorithmically random: Its algorithmic complexity is of the order of its length (Li and Vitànyi, 1994):

$$
K\left(\left\langle\mathcal{E}_{n} \mid+,-\right\rangle\right) \simeq n
$$

By contrast, algorithmic complexity of the measurement outcomes in the $\{|0\rangle,|1\rangle\}$ basis will be small:

$$
K\left(\left\langle\mathcal{E}_{n} \mid 0,1\right\rangle\right) \ll n,
$$

since the outcomes will be either $00 \ldots 0$ or $11 \ldots 1$. Observer seeking preferred states of the system by looking at the environment should then search for the minimal record size and, thus, for the maximum redundancy in the environmental record. States of the system that are recorded redundantly in the environment must have survived repeated instances of the environment monitoring, and are obviously robust and predictable.

Predictability we have utilized before to devise a "sieve" to select preferred states is used here again, but in a different guise: Rather than search for predictable sets of states of the system we are now looking for the records of the states of the system in the environment. Sequences of states of environment subsystems correlated with pointer states are mutually predictable and, hence, collectively algorithmically simple. States that are predictable in spite of the interactions with the environment are also easiest to predict from their impact on its state.

The state of the form of Eq. (7.1) can serve as an example of amplification. Generation of redundancy through amplification brings about objective existence of the otherwise subjective quantum states. States $|\uparrow\rangle$ and $|\downarrow\rangle$ of the system can be found out reliably from a small fraction of the environment. By contrast, to find out whether the system was in a state $|\odot\rangle$ or $|\otimes\rangle$ one would need to detect all of the environment. Objectivity can be defined as the ability of many observers to reach consensus independently. Such consensus concerning states $|\uparrow\rangle$ and $|\downarrow\rangle$ is easily established - many $(\sim N / n)$ can independently measure fragments of the environment.

\section{Action distance}

One measure of robustness of the environmental records is the action distance (Zurek, 1998a). It is given by the total action necessary to undo the distinction between the states of the environment corresponding to different states of the system, subject to the constraints arising from the fact that the environment consists of subsystems. Thus, to obliterate the difference between $\left|\mathcal{E}_{\uparrow}\right\rangle$ and $\left|\mathcal{E}_{\downarrow}\right\rangle$ in Eq. (7.1), one needs to "flip" one-by-one $N$ subsystems of the environment. That implies an action - i.e., the least total angle by which a states must be rotated, see Section IIB - of:

$$
\Delta\left(\left|\mathcal{E}_{\uparrow}\right\rangle,\left|\mathcal{E}_{\downarrow}\right\rangle\right)=N\left[\frac{\pi}{2} \cdot \hbar\right] .
$$

By contrast a "flip" of phase of just one bit will reverse the correspondence between the states of the system and of the environment superpositions that make up $\left|\mathcal{E}_{\odot}\right\rangle$ and $\left|\mathcal{E}_{\otimes}\right\rangle$ in $\operatorname{Eq}(7.2)$. Hence:

$$
\Delta\left(\left|\mathcal{E}_{\odot}\right\rangle,\left|\mathcal{E}_{\otimes}\right\rangle\right)=1\left[\frac{\pi}{2} \cdot \hbar\right] .
$$

Given a fixed division of the environment into subsystems the action distance is a metric on the Hilbert space (Zurek, 1998a). That is;

$$
\begin{gathered}
\Delta(|\psi\rangle,|\psi\rangle)=0, \\
\Delta(|\psi\rangle,|\varphi\rangle)=\Delta(|\varphi\rangle,|\psi\rangle) \geq 0,
\end{gathered}
$$

and the triangle inequality:

$$
\Delta(|\psi\rangle,|\varphi\rangle)+\Delta(|\varphi\rangle,|\gamma\rangle) \geq \Delta(|\psi\rangle,|\gamma\rangle)
$$

are all satisfied. 
In defining $\Delta$ it is essential to restrict rotations to the subspaces of the subsystems of the whole Hilbert space, and to insist that the unitary operations used in defining distance act on these subspaces. It is possible to relax constraints on such unitary operations by allowing, for example, pairwise or even more complex interactions between subsystems. Clearly, in absence of any restrictions the action required to rotate any $|\psi\rangle$ into any $|\varphi\rangle$ would be no more than $\frac{\pi}{2} \hbar$. Thus, constraints imposed by the natural division of the Hilbert space of the environment into subsystems play an essential role. Preferred states of the system can be sought by extremizing action distance between the corresponding record states of the environment. In simple cases (e.g., see "bit-bybyte", Eq. (4.7) and below) the action distance criterion for preferred states coincides with the predictability sieve definition (Zurek, 1998a).

\section{Redundancy and mutual information}

The most direct measure of reliability of the environment as a witness is the information-theoretic redundancy of einselection itself. When environment monitors the system (see Fig. 4), the information about its state will spread to more and more subsystems of the environment. This can be represented by the state vector $\left|\psi_{\mathcal{S E}}\right\rangle$, Eq. (7.1), with increasingly long sequences of 0's and 1 's in the record states. The record size - the number $N$ of the subsystems of the environment involved - does not affect the density matrix of the system $\mathcal{S}$. Yet, it obviously changes accessibility abd robustness of the information analogues of the Darwinian "fitness". As an illustration, let us consider c-shift's. One subsystem of the environment (say, $\mathcal{E}_{1}$ ) with the dimension of the Hilbert space no less than that of the system

$$
\operatorname{Dim}_{\mathcal{E}_{1}} \geq \operatorname{Dim}_{\mathcal{S}}
$$

suffices to eradicate off-diagonal elements of $\rho_{\mathcal{S}}$ in the control basis. On the other hand, when $N$ subsystems of the environment correlate with the same set of states of $\mathcal{S}$, the information about these states is simultaneously accessible more widely. While $\rho_{\mathcal{S}}$ is no longer changing, spreading of the information makes the existence of the pointer states of $\mathcal{S}$ more objective - they are easier to discover without being perturbed.

Information theoretic redundancy is defined as the difference between the least number of bits needed to uniquely specify the message and the actual size of the encoded message. Extra bits allow for detection and correction of errors (Cover and Thomas, 1991). In our case, the message is the state of the system, and the channel is the environment. The information about the system will often spread over all of the Hilbert space $\mathcal{H}_{\mathcal{E}}$ that is enormous compared to $\mathcal{H}_{\mathcal{S}}$. Redundancy of the record of the pointer observables of selected systems can be also huge. Moreover, typical environments consist of obvious subsystems (i.e., photons, atoms, etc.). It is then useful to define redundancy of the record by the number of times the information about the system has been copied, or by how many times it can be independently extracted from the environment.

In the simple example of Eq. (7.1) such redundancy ratio $\mathcal{R}$ for the $\{|\uparrow\rangle,|\downarrow\rangle\}$ basis will be given by $N$, the number of environment bits perfectly correlated with the obviously preferred basis of the system. More generally, but in the same case of perfect correlation we obtain:

$$
\mathcal{R}=\frac{\lg \left(\operatorname{Dim\mathcal {H}}_{\mathcal{E}}\right)}{\lg \left(\operatorname{Dim\mathcal {H}}_{\mathcal{S}}\right)}=\log _{\operatorname{Dim\mathcal {H}}_{\mathcal{S}}}\left(\operatorname{Dim}_{\mathcal{E}}\right)=N
$$

where $\mathcal{H}_{\mathcal{E}}$ is the Hilbert space of the environment perfectly correlated with the pointer states of the system.

On the other hand, with respect to the $\{|\odot\rangle,|\otimes\rangle\}$ basis, the redundancy ratio for $\left|\psi_{\mathcal{S E}}\right\rangle$ of Eq. (7.2) is only $\sim 1$ (see also Zurek, 1983; 2000): Redundancy measures the number of errors that can obliterate the difference between two records, and in this basis one phase flip is clearly enough. This basis dependence of redundancy suggests an alternative strategy to seek preferred states.

To define $\mathcal{R}$ in general we can start with mutual information between the subsystems of the environment $\mathcal{E}_{k}$ and the system $\mathcal{S}$. As we have already seen in section IV, definition of mutual information in quantum mechanics is not straightforward. The basis-independent formula:

$$
\mathcal{I}_{k}=\mathcal{I}\left(\mathcal{S}: \mathcal{E}_{k}\right)=H(\mathcal{S})+H\left(\mathcal{E}_{k}\right)-H\left(\mathcal{S}, \mathcal{E}_{k}\right)
$$

is simple to evaluate (although it does have some strange features; see Eqs. (4.30) - (4.36)). In the present context it involves joint density matrix:

$$
\rho_{\mathcal{S} \mathcal{E}_{k}}=\operatorname{Tr} r_{\mathcal{E} / \mathcal{E}_{k}} \rho_{\mathcal{S E}}
$$

where the trace is carried out over all of the environment except for its singled out fragment $\mathcal{E}_{k}$. In the example of Eq. (7.1) for any of the environment bits

$$
\rho_{\mathcal{S} \mathcal{E}_{k}}=|a|^{2}|\uparrow\rangle\langle\uparrow|| 0\rangle\left\langle\left. 0|+| b\right|^{2} \mid \downarrow\right\rangle\langle\downarrow|| 1\rangle\langle 1| .
$$

Given the partitioning of the environment into subsystems, the redundancy ratio can be defined as:

$$
\mathcal{R}_{\mathcal{I}\left(\left\{\otimes \mathcal{H}_{\mathcal{E}_{k}}\right\}\right)}=\sum_{k} \mathcal{I}\left(\mathcal{S}: \mathcal{E}_{k}\right) / H(\mathcal{S})
$$

When $\mathcal{R}$ is maximized over all of the possible partitions,

$$
\mathcal{R}_{\mathcal{I} \max }=R_{\left\{\otimes \mathcal{H}_{\mathcal{E}_{k}}\right\}}
$$

obtains. Roughly speaking, and in the case when the number of the environment subsystems is large, $\mathcal{R}_{\mathcal{I}_{\text {max }}}$ is the total number of copies of the information about (the optimal basis of) $\mathcal{S}$ that exist in $\mathcal{E}$. Maximal redundancy ratio $\mathcal{R}_{\mathcal{I}_{\text {max }}}$ is of course basis - independent.

The information defined through the symmetric $\mathcal{I}_{k}$, Eq. (7.11), is in general inaccessible to observers who interrogate environment one subsystem at a time (Zurek, 
2002a). It makes therefore a lot of sense to consider the basis-dependent locally accessible information and define the corresponding redundancy ratio $\mathcal{R}_{\mathcal{J}}$ using:

$$
\mathcal{J}_{k}=\mathcal{J}\left(\mathcal{S}: \mathcal{E}_{k}\right)=H(\mathcal{S})+H\left(\mathcal{E}_{k}\right)-\left(H(\mathcal{S})+H\left(\mathcal{E}_{k} \mid \mathcal{S}\right)\right) .
$$

Conditional entropy must be computed in a specific basis of the system (see Eq. (4.32)). All of the other steps that have led to the definition of $\mathcal{R}_{\mathcal{I} \max }$ can be now repeated using $\mathcal{J}_{k}$. In the end, a basis - dependent:

$$
\mathcal{R}_{\mathcal{J}}(\{|s\rangle\})=\mathcal{R}_{\mathcal{J}}\left(\otimes \mathcal{H}_{\mathcal{E}_{k}}\right)
$$

obtains. $\mathcal{R}_{\mathcal{J}}(\{|s\rangle\})$ quantifies the mutual information between the collection of subsystems $\mathcal{H}_{\mathcal{E}_{k}}$ of the environment and the basis $\{|s\rangle\}$ of the system. We note that the condition of non-overlapping partitions guarantees that all of the corresponding measurements commute, and that the information can be indeed extracted independently from each environment fragment $\mathcal{E}_{k}$.

Preferred basis of $\mathcal{S}$ can be now defined by maximizing $\mathcal{R}_{\mathcal{J}}(\{|s\rangle\})$ with respect to the selection of $\{|s\rangle\}$ :

$$
\mathcal{R}_{\mathcal{J} \text { max }}=\max _{\{|s\rangle\} ;\left\{\otimes \mathcal{H}_{\mathcal{E}_{k}}\right\}} \mathcal{R}_{\mathcal{J}}(\{|s\rangle\})
$$

This maximum can be sought either by varying the basis of the system only (as it is indicated above) or by varying both the basis and the partition of the environment.

It remains to be seen whether and under what circumstances pointer basis "stands out" through its definition in terms of $\mathcal{R}_{\mathcal{J}}$. The criterion for a well - defined set of pointer states $\{|p\rangle\}$ would be:

$$
\mathcal{R}_{\mathcal{J} \text { max }}=\mathcal{R}_{\mathcal{J}}(\{|p\rangle\}) \gg \mathcal{R}_{\mathcal{J}}(\{|s\rangle\})
$$

where $\{|s\rangle\}$ are typical superpositions of states belonging to different pointer eigenstates.

This definition of preferred states directly employs the notion of multiplicity of records available in the environment. Since $\mathcal{J} \leq \mathcal{I}$, it follows that:

$$
\mathcal{R}_{\mathcal{J} \text { max }} \leq \mathcal{R}_{\mathcal{I} \text { max }}
$$

The important feature of either version of $\mathcal{R}$ that makes them useful for our purpose is their independence on $H(\mathcal{S})$ : The dependence on $H(\mathcal{S})$ is in effect "normalized out" of $\mathcal{R}$. $\mathcal{R}$ characterizes the "fan-out" of the information about the preferred basis throughout environment, without a reference to what is known about the system. The usual redundancy (in bits) is then $\sim \mathcal{R} \cdot \mathcal{H}(\mathcal{S})$, although other implementations of this program (Ollivier, Poulin, and Zurek, 2002) employ different measures of redundancy, which may be even more specific than the redundancy ratio we have described above. Indeed, what is important here is the genreal idea of measuring classicality of quantum states through the number of copies they imprint throughout the Universe. This is a very Darwinian approach: We define classicality related to einselection in ways reminiscent of 'fitness' in natural selection: States that spawn most of the (informationtheoretic) progeny are the most classical.

\section{Redundancy ratio rate}

The rate of change of redundancy is of interest as another measure of 'fitness', perhaps closest to the definitions of fitness used in modeling natural selection. Redundancy can increase either as a result of interactions between the system and the environment, or because the environment already correlated with $\mathcal{S}$ is passing on the information to more distant environments. In this second case 'genetic information' is passed on by the 'progeny' of the original state. Even an observer consulting the environment becomes a part of such a more distant environment. Redundancy rate is defined as:

$$
\dot{\mathcal{R}}=\frac{d}{d t} \mathcal{R}
$$

Either basis-dependent or basis-independent versions of $\dot{\mathcal{R}}$ may be of interest.

In general, it may not be easy to compute either $\mathcal{R}$ or $\dot{\mathcal{R}}$ exactly. This is nevertheless possible in models (such as those leading to Eqs. (7.1) - (7.2)). The simplest illustrative example corresponds to the c-not model of decoherence in Fig. 4. One can imagine that the consecutive record bits get correlated with the two branches (corresponding to $|0\rangle$ and $|1\rangle$ in the "control") at discrete moments of time. $\mathcal{R}(t)$ would be then the total number of c-nots that have acted over the time $t$, and $\dot{\mathcal{R}}$ is the number of new c-nots added per unit time.

Redundancy rate measures information flow from the system to the environment. Note that, after the first c-not in the example of Eqs. (7.1) - (7.2), $\mathcal{R}_{\mathcal{I}}$ will jump immediately from 0 to 2 bits, while the basis-specific $\mathcal{R}_{\mathcal{J}}$ will increase from 0 to 1 . In our model this initial discrepancy (which reflects quantum discord, Eq. (4.36), between $\mathcal{I}$ and $\mathcal{J}$ ) will disappear after the second c-not.

Finally, we note that $\mathcal{R}$ and, especially, $\dot{\mathcal{R}}$ can be used to introduce new predictability criteria: The states (or the observables) that are being recorded most redundantly are the obvious candidates for the "objective", and therefore for the "classical".

\section{B. Observers and the Existential Interpretation}

Von Neumann (1932), London and Bauer (1939) and Wigner (1963) have all appealed to the special role of the conscious observer. Consciousness was absolved from following unitary evolution, and, thus, could collapse the wavepacket. Quantum formalism has led us to a different view, that nevertheless allows for a compatible conclusion. In essence, macroscopic systems are open, and their evolution is almost never unitary. Records maintained by the observers are subject to einselection. In a binary alphabet decoherence will allow only for the two logical states, and prohibit their superpositions (Zurek, 1991). For human observers neurons conform to this binary convention and the decoherence times are short 
(Tegmark, 2000). Thus, even if a cell of the observer entangles through a premeasurement with a pure quantum state, the record will become effectively classical almost instantly: As a result, it will be impossible to "read it off" in any basis except for the einselected one. This censorship of records is the key difference between the existential interpretation and the original Everett's MWI.

Decoherence treats observer as any other macroscopic system. There is, however, one feature distinguishing observers from the rest of the Universe: They are aware of the content of their memory. Here we are using aware in a down - to - earth sense: Quite simply, observers know what they know. Their information processing machinery (that must underlie higher functions of the mind such as "consciousness") can readily consult the content of their memory.

The information stored in the memory comes with 'strings attached'. The physical state of the observer is described in part by the data in his records. There is no information without representation. The information observer has could be, in principle, deduced from his physical state. Observer is - in part - information. Moreover, this information encoded in states of macroscopic quantum systems (neurons) is by no means secret: As a result of lack of isolation the environment - having redundant copies of the relevant data - 'knows' in detail everything observer knows. Configurations of neurons in our brains, while at present undecipherable, are in principle as objective and as widely accessible as the information about the states of other macroscopic objects.

The observer $i s$ what he knows. In the unlikely case of a flagrantly quantum input the physical state of the observers memory will decohere, resulting almost instantly in the einselected alternatives, each of them representing simultaneously both observer and his memory. The 'advertising' of this state throughout the environment makes it effectively objective.

An observer perceiving the Universe from within is in a very different position than an experimental physicist studying a state vector of a quantum system. In a laboratory, Hilbert space of the investigated system is typically tiny. Such systems can be isolated, so that often the information loss to the environment can be prevented. Then the evolution is unitary. The experimentalist can know everything there is to know about it. Common criticisms of the approch advocated in this paper are based on an unjustified extrapolation of the above laboratory situation to the case of the observer who is a part of the Universe. Critics of decoherence often note that the differences between the laboratory example above and the case of the rest of the Universe are 'merely quantitative' - the system under investigation is bigger, etc. So why cannot one analyze - they ask - interactions of the observer and the rest of the Universe as before, for a small isolated quantum system?

In the context of the existential interpretation the analogy with the laboratory is, in effect, turned "upside down": For, now the observer (or the apparatus, or any- thing effectively classical) is continuously monitored by the rest of the Universe. Its state is repeatedly collapsed - forced into the einselected states - and very well (very redundantly) 'known' to the rest of the Universe.

The 'higher functions' of observers - e.g., consciousness, etc. - may be at present poorly understood, but it is safe to assume that they reflect physical processes in the information processing hardware of the brain. Hence, mental processes are in effect objective, as they leave an indelible imprint on the environment: The observer has no chance of perceiving either his memory, or any other macroscopic part of the Universe in some arbitrary superposition. Moreover, memory capacity of observers is miniscule compared to information content of the Universe. So, while observers may know exact state of laboratory systems, their records of the Universe will be very fragmentary. By contrast, the Universe has enough memory capacity to acquire and maintain detailed records of states of macroscopic systems and their histories.

\section{Events, Records, and Histories}

Suppose that instead of a monotonous record sequence in the environment basis corresponding to the pointer states of the system $\{|\uparrow\rangle,|\downarrow\rangle\}$ implied by Eq. (7.1) the observer looking at the environment detects:

$$
000 \ldots 0111 \ldots 1000 \ldots 0111 \ldots
$$

Given appropriate additional assumptions, such sequences consisting of long stretches of record 0's and 1's justify inference of the history of the system. Let us further assume that observer's records come from intercepting a small fragment of the environment. Other observers will be then able to consult their independently accessible environmental records, and will infer (more or less) the same history. Thus, in view of the "preponderance of evidence" history defined as a sensible inference from the available records can be probed by many independently, and can be regarded as classical and objective.

The redundancy ratio of the records $\mathcal{R}$ is a measure of this objectivity. Note that this relatively objective existence (Zurek, 1998a) is an operational notion, quantified by the number of times the state of the system can be found out independently, and not some "absolute objectivity". However, and in a sense that can be rigorously defined, relative objectivity tends to absolute objectivity in the limit $\mathcal{R} \longrightarrow \infty$. For example, cloning of unknown states becomes possible (Bruss, Ekert, and Macchiavello, 1999, Jozsa, 2002) in spite of the "no cloning" theorem (Wootters and Zurek, 1982, Dieks 1982). In that limit, and given same reasonable constraints on the nature of the interactions and on the structure of the environment which underlie the definition of $\mathcal{R}$, it would take infinite resources such as action, Eqs. (7.5) - (7.9), to hide or subvert evidence of such an objective history.

There are differences and parallels between relatively objective histories introduced here and consistent histo- 
ries proposed by Griffiths $(1984,1996)$, and investigated by Gell-Mann and Hartle (1990; 1993; 1997), Omnès (1988; 1992; 1994), Halliwell (1999), and others (Dowker and Kent, 1996; Kiefer, 1996). Such histories are defined as time-ordered sequences of projection operators $P_{\alpha_{1}}^{1}\left(t_{1}\right), P_{\alpha_{2}}^{2}\left(t_{2}\right), \ldots, P_{\alpha_{n}}^{n}\left(t_{n}\right)$ and are abbreviated $\left[P_{\alpha}\right]$. Consistency is achieved when they can be combined into coarse grained sets (where the projectors defining coarse - grained set are given by sums of the projectors in the original set) while obeying probability sum rules: Probability of a bundle of histories should be a sum of the probabilities of the constituent histories. The corresponding condition can be expressed in terms of the decoherence functional (Gell-Mann and Hartle, 1990);

$D\left(\left[P_{\alpha}\right],\left[P_{\beta}\right]\right)=\operatorname{Tr}\left(\left(P_{\alpha_{n}}^{n}\left(t_{n}\right) \ldots P_{\alpha_{1}}^{1}\left(t_{1}\right) \rho P_{\beta_{1}}^{1}\left(t_{1}\right) \ldots P_{\beta_{n}}^{n}\left(t_{n}\right)\right)\right.$ $(7.21)$

Above, the state of the system of interest is described by the density matrix $\rho$. Griffiths' condition is equivalent to the vanishing of the real part of the expression above, $\operatorname{Re}\left\{D\left(\left[P_{\alpha}\right],\left[P_{\beta}\right]\right\}=p_{\alpha} \delta_{\alpha, \beta}\right.$. As Gell-Mann and Hartle (1990) emphasize, it is more convenient - and in the context of and emergent classicality more realistic to demand instead $D\left(\left[P_{\alpha}\right],\left[P_{\beta}\right]\right)=p_{\alpha} \delta_{\alpha, \beta}$. Both weaker and stronger conditions for the consistency of histories were considered (Goldstein and Page, 1995; Gell-Mann and Hartle, 1997). The problem with all of them is that the resulting histories are very subjective: Given an initial density matrix of the Universe it is in general quite easy to specify many different, mutually incompatible consistent sets of histories. This subjectivity leads to serious interpretational problems (d'Espagnat, 1989; 1995; Dowker and Kent, 1996). Thus, a demand for exact consistency as one of the conditions for classicality is both uncomfortable (overly restrictive) and insufficient (as the resulting histories are very non-classical). Moreover, coarse-grainings that help secure approximate consistency have to be, in effect, guessed.

The attitudes adopted by the practitioners of the consistent histories approach in view of its unsuitability for the role of the cornerstone of the emergent classicality differ. Initially - before difficulties became apparent - it was hoped that such approach would answer all of the interpretational questions, perhaps when supplemented by a subsidiary condition, i.e. some assumption about favored coarse-grainings. At present, some still aspire to the original goals of deriving classicality from consistency alone. Others may uphold the original aims of the program, but they also generally rely on environmentinduced decoherence, using in calculations variants of models we have presented in this paper. This strategy has been quite successful - after all, decoherence leads to consistency. For instance, special role of the hydrodynamic observables (Gell-Mann and Hartle, 1990; Dowker and Halliwell, 1992; Halliwell, 1999; Brun and Hartle, 1999) can be traced to their predictability, or to their approximate commutativity with the total Hamiltonian (see Eq. (4.41)). On the other hand, the original goals of Griffiths $(1984,1996)$ have been more modest: Using consistent histories, one can discuss sequences of events in an evolving quantum system without logical contradictions. The "golden middle" is advocated by Griffiths and Omnès (1999) who regard consistent histories as a convenient language, rather than as an explanation of classicality.

The origin of the effective classicality can be traced to decoherence and einselection. As was noted by GellMann and Hartle (1990), and elucidated by Omnès (1992; 1994) decoherence suffices to ensure approximate consistency. But consistency is both not enough and too much - it is too easy to accomplish, and does not necessarily lead to classicality (Dowker and Kent, 1996). What is needed instead is the objectivity of events and their timeordered sequences - histories. As we have seen above, both can appear as a result of einselection.

\section{Relatively Objective Past}

We have already provided an operational definition of relatively objective existence of quantum states. It is easy to apply it to events and histories: When many observers can independently gather compatible evidence concerning an event, we call it relatively objective. Relatively objective history is then a time-ordered sequence of relatively objective events.

Monitoring of the system by the environment leads to decoherence and einselection. It will also typically lead to redundancy and hence effectively objective classical existence in the sense of 'quantum Darwinism'. Observers can independently access redundant records of events and histories imprinted in the environmental degrees of freedom. The number of observers who can examine evidence etched in the environment can be of the order of, and is bounded from above by $\mathcal{R}_{\mathcal{J}}$. Redundancy is a measure of this objectivity and classicality.

As observers record their data, $\mathcal{R}_{\mathcal{J}}$ changes: Consider an observer who measures the 'right observable' of $\mathcal{E}$ (i.e., the one with the eigenstates $|0\rangle,|1\rangle$ in the example of Eq. (7.1)). Then his records and - as his records decohere, their environment - become a part of evidence, and are correlated with the preferred basis of the system. Consequently, $\mathcal{R}_{\mathcal{J}}$ computed from Eq. (7.14) increases. Every interaction that increases the number of the records also increases $\mathcal{R}_{\mathcal{J}}$. This is obvious for the "primary" interactions with the system, but it is also true for the secondary, tertiary, etc. acts of replication of the information obtained from the observers who recorded primary state of the system, from the environment, from the environment of the environment, and so on.

A measurement reveals to the observer "his" branch of the universal state vector. The correlations established alter observer's state - his records - and "attach" him to this branch. He will share it with other observers who examined the same set of observables, and who have recorded compatible results.

It is also possible to imagine a stubborn observer who 
insists on measuring either the relative phase between the two obvious branches of the environment in Eq. (7.2), or the state of the environment in the Hadamardtransformed basis $\{|+\rangle,|-\rangle\}$. In either case the distinction between the two outcomes could determine the state of the spin in the $\{|\odot\rangle,|\otimes\rangle\}$ basis. However, in that basis $\mathcal{R}_{\mathcal{J}}=1$. Hence, while in principle these measurements can be carried out and yield the correct result, the information concerning $\{|\odot\rangle,|\otimes\rangle\}$ basis is not redundant, and, therefore, not objective: Only one stubborn observer can access it directly. As a result $\mathcal{R}_{\mathcal{J}}$ will decrease. Whether the $\mathcal{R}_{\mathcal{J}}(\{|\odot\rangle,|\otimes\rangle\})$ will become larger than $\mathcal{R}_{\mathcal{J}}(\{|\uparrow\rangle,|\downarrow\rangle\})$ was before the measurement of the stubborn observer will depend on detailed comparison of the initial redundancy with the amplification involved, decoherence, etc.

There is a further significant difference between the two stubborn observers considered above. When the observer measures the phase between the two sequences of 0 's and 1's in Eq. (7.2), correlations between the bits of the environment remain. Thus, even after his measurement one could find relatively objective evidence of the past event - past state of the spin - and, in more complicated cases, of the history. On the other hand, measurement of all the environment bits in the $\{|+\rangle,|-\rangle\}$ basis will obliterate evidence of such a past.

Relatively objective existence of events is the strongest condition we have considered here. It is a consequence of the existence of multiple records of the same set of states of the system. It allows for such manifestations of classicality as unimpeded cloning. It implies einselection of states most closely monitored by the environment. Decoherence is clearly weaker and easier to accomplish.

"The past exists only insofar as it is recorded in the present" (dictum often repeated by Wheeler) may the best summary of the above discussion. Relatively objective reality of few selected observables in our familiar Universe is measured by their fitness - by the redundancy with which they are recorded in the environment. This multiplicity of available copies of the same information can be regarded as a consequence of amplification, and as a cause of indelibility. Multiple records safeguard objectivity of our past.

\section{DECOHERENCE IN THE LABORATORY}

The biggest obstacle in the experimental study of decoherence is - paradoxically - its effectiveness. In the macroscopic domain only the einselected states survive. Their superpositions are next to impossible to prepare. In the mesoscopic regime one may hope to adjust the size of the system, and, thus, interpolate between quantum and classical. The strength of the coupling to the environment is the other parameter one may employ to control the decoherence rate.

One of the key consequences of monitoring by the environment is the inevitable introduction of the Heisen- berg uncertainty into the observable complementary to the one that is monitored. One can simulate such uncertainty without any monitoring environment by introducing classical noise. In each specific run of the experiment - for each realization of time-dependent noise - quantum system will evolve deterministically. However, after averaging over different noise realizations evolution of the density matrix describing an ensemble of systems may approximate decoherence due to an entangling quantum environment. In particular, the master equation may be essentially the same as for true decoherence, although the interpretational implications are more limited. Yet, using such strategies one can simulate much of the dynamics of open quantum systems.

The strategy of simulating decoherence can be taken further: Not just the effect of the environment, but also the dynamics of the quantum system can be simulated by classical means. This can be accomplished when classical wave phenomena follow equations of motion related to Schrödinger equation. We shall discuss experiments that fall into all of the above categories.

Last not least, while decoherence - through einselection - helps solve the measurement problem, it is also a major obstacle to quantum information processing. We shall thus end this section briefly describing strategies that may allow one to tame decoherence.

\section{A. Decoherence due to entangling interactions}

Several experiments fit this category, and more have been proposed. Decoherence due to emission or scattering of photons has been investigated by the MIT group (Chapman et al., 1995; Kokorowski et al., 2001) using atomic interferometry. Emission or scattering deposits a record in the environment. It can store information about the path of the atom providing photon wavelength is shorter than the separation between the two of the atoms. In case of emission this record is not redundant, as the atom and photon are simply entangled $-\mathcal{R}_{\mathcal{J}} \sim 1$ in any basis. Scattering may involve more photons, and the recent careful experiment has confirmed the saturation of decoherence rate at distances in excess of photon wavelength (Gallis and Fleming, 1990; Anglin, Paz, and Zurek, 1997).

There is an intimate connection between interference and complementarity in the two-slit experiment on one hand, and the entanglement on the other (Wootters and Zurek, 1979). Consequently, appropriate measurements of the photon allow one to restore interference fringes in the conditional subensembles corresponding to a definite phase between the two photon trajectories (see especially Chapman et al. (1995), as well as Kwiat, Steinberg and Chiao (1993); Pfau et al., 1994; Herzog, Kwiat, Weinfurter and Zeilinger, (1995) for implementations of this "quantum erasure" trick due to Hillery and Scully (1983)). Similar experiments have been also carried out using neutron interferometry (see e.g. Rauch, (1998)). 
In all of these experiments one is dealing with a very simplified situation involving a single microsystem and a single "unit" of decoherence $\left(\mathcal{R}_{\mathcal{J}} \sim 1\right)$ caused by a single quantum of the environment. Experiments on a mesoscopic system monitored by the environment are obviously much harder to devise. Nevertheless, Sergé Haroche, Jean-Michel Raimond, Michel Brune and their colleagues (Brune et al., 1996; Haroche 1998, Raimond, Brune, and Haroche, 2001) have carried out a spectacular experiment of this type, yielding solid evidence in support of the basic tenets of the environment-induced transition from quantum to classical. Their system is a microwave cavity. It starts in a coherent state with an amplitude corresponding to a few photons.

"Schrödinger cat" is created by introducing an atom in a superposition of two Rydberg states, $|+\rangle=|0\rangle+|1\rangle$ : The atom passing through the cavity puts its refractive index in a superposition of two values. Hence, the phase of the coherent state shifts by the amount correlated with the state of the atom, creating an entangled state:

$$
|\rightarrow\rangle(|0\rangle+|1\rangle) \Longrightarrow|\nearrow\rangle|0\rangle+|\searrow\rangle|1\rangle=|\vartheta\rangle
$$

Arrows indicate relative phase space locations of coherent states. States of the atom are $|0\rangle$ and $|1\rangle$. "Schrödinger kitten" is prepared from this entangled state by measuring the atom in the $\{|+\rangle,|-\rangle\}$ basis:

$$
|\vartheta\rangle=(|\nearrow\rangle+|\searrow\rangle)|+\rangle+(|\nearrow\rangle-|\searrow\rangle)|-\rangle
$$

Thus, atom in the state $|+\rangle$ implies preparation of a "positive cat" $|\uplus\rangle=|\nearrow\rangle+|\searrow\rangle$ in the cavity. Such superpositions of coherent states could survive forever if there was no decoherence. However, radiation leaks out of the cavity. Hence, environment acquires information about the state inside. Consequences are tested by passing another atom in the state $|+\rangle=|0\rangle+|1\rangle$ through the cavity. In absence of decoherence the state would evolve as:

$$
\begin{array}{r}
|\uplus\rangle|+\rangle=(|\nearrow\rangle+|\searrow\rangle)(|0\rangle+|1\rangle) \Longrightarrow \\
(|\uparrow\rangle|0\rangle+|\rightarrow\rangle|1\rangle)+(|\rightarrow\rangle|0\rangle+|\downarrow\rangle|1\rangle) \\
\quad=(|\uparrow\rangle|0\rangle+|\downarrow\rangle|1\rangle)+\sqrt{2}|\rightarrow\rangle|+\rangle .
\end{array}
$$

Above we have omitted the overall normalization, but retained the (essential) relative amplitude.

For the above state detection of $|+\rangle$ in the first (preparatory) atom implies the conditional probability of detection of $|+\rangle, p_{+\mid+}=\frac{3}{4}$, for the second (test) atom. Decoherence will suppress off-diagonal terms of the density matrix, so that, some time after the preparation, $\rho_{\text {cavity }}$ that starts, say, as $|\uplus\rangle\langle\uplus|$ becomes:

$$
\begin{aligned}
\rho_{\text {cavity }} & =(|\nearrow\rangle\langle\nearrow|+| \searrow\rangle\langle\searrow|) / 2 \\
& +z(|\nearrow\rangle\langle\searrow|+| \searrow\rangle\langle\nearrow|) / 2 .
\end{aligned}
$$

When $z=0$ the conditional probability is $p(+\mid+)=\frac{1}{2}$.

In the intermediate cases intermediate values of this and other relevant conditional probabilities are predicted. The rate of decoherence, and, consequently, the timedependent value of $z$ can be estimated from the cavity quality factor $Q$, and from the data about the coherent state initially present in the cavity. Decoherence rate is a function of separation of the two components of the cat $|\uplus\rangle$. Experimental results agree with predictions.

The discussion above depends on the special role of coherent states. Coherent states are einselected in harmonic oscillators, and, hence, in the underdamped bosonic fields (Anglin and Zurek, 1996). Thus, they are the pointer states of the cavity. Their special role is recognized implicitly above: If number eigenstates were einselected, predictions would be obviously quite different. Therefore, while the ENS experiment is focused on decoherence rate, confirmation of the predicted special role of coherent states in bosonic fields is its important (albeit implicit) corollary.

\section{B. Simulating decoherence with classical noise}

¿From the fundamental point of view, the distinction between cases when decoherence is caused by entangling interactions with the quantum state of the environment and when it is simulated by a classical noise in the observable complementary to the "pointer" is essential. However, from the engineering point of view (adopted, e.g., by the practitioners of quantum computation, see Nielsen and Chuang, 2000 for discussion) this may not matter. For instance, quantum error correction techniques (Shor, 1995; Steane, 1996; Preskill, 1999) are capable of dealing with either. Moreover, experimental investigations of this subject often involve both.

The classic experiment in this category was carried out recently by David Wineland, Chris Monroe, and their collaborators (Myatt et al, 2000; Turchette et al., 2000). They use ion trap to study behavior of individual ions in a "Schrödinger cat" state (Monroe et al., 1996) under the influence of injected classical noise. They also embark on a preliminary study of "environment engineering".

Superpositions of two coherent states as well as of number eigenstates were subjected to simulated high temperature amplitude and phase "reservoirs". This was done through time-dependent modulation of the selfHamiltonian of the system. For the amplitude noise these are in effect random fluctuations of the location of the minimum of the harmonic trap. Phase noise corresponds to random fluctuations of the trap frequency.

In either case, the resulting loss of coherence is well described by the exponential decay with time, with the exponent that scales with the square of the separation between the two components of the macroscopic quantum superposition (e.g., Eq. (5.34)). The case of the amplitude noise approximates decoherence in quantum Brownian motion in that the coordinate is monitored by the environment, and, hence, the momentum is perturbed. (Note that in the underdamped harmonic oscillator rotating wave approximation blurs the distinction between $x$ and $p$, leading to einselection of coherent states.) The phase noise would arise in an environment monitoring the 
number operator, thus leading to uncertainty in phase. Consequently, number eigenstates are einselected.

The applied noise is classical, and the environment does not acquire any information about the ion $\left(\mathcal{R}_{\mathcal{I}}=0\right)$. Thus, following a particular realization of the noise the state of the system is still pure. Nevertheless, an ensemble average over many noise realizations is represented by the density matrix that follows an appropriate master equation. Thus, as Wineland, Monroe, and their colleagues note, decoherence simulated by the classical noise could be in each individual case - for each realisation - reversed by simply measuring the corresponding timedependent noise run either beforehand or afterwards, and then applying the appropriate unitary transformation to the state of the system. By contrast, in the case of entangling interactions, two measurements - one preparing the environment before the interaction with the environment, the other following it - would be the least required for a chance of undoing the effect of decoherence.

The same two papers study decay of a superposition of number eigenstates $|0\rangle$ and $|2\rangle$ due to an indirect coupling with the vacuum. This proceeds through entanglement with the first order environment (that, in effect, consists of the other states of the harmonic oscillator) and a slower transfer of information to the distant environment. Dynamics involving the system and its first - order environment leads to non-monotonic behavior of the off-diagonal terms representing coherence. Further studies of decoherence in the ion trap setting are likely to follow, as this is an attractive implementation of the quantum computer (Cirac and Zoller, 1995).

\section{Decoherence, Noise, and Quantum Chaos}

Following a proposal of Graham, Schlautmann, and Zoller (1993) Mark Raizen and his group (Moore et al., 1994) used a one-dimensional optical lattice to implement a variety of 1-D chaotic systems including the "standard map". Various aspects of the behavior expected from a quantized version of a classically chaotic system were subsequently found, including, in particular, dynamical localization (Casati and Chirikov, 1995a; Reichl, 1992).

Dynamical localization establishes, in a class of driven quantum chaotic systems, a saturation of momentum dispersion, and leads to a characteristic exponential form of its distribution (Casati and Chirikov, 1995a). Localization is obviously a challenge to the quantum-classical correspondence, since in these very same systems classical prediction has the momentum dispersion growing unbounded, more or less with the square root of time. However, it sets in after $t_{L} \sim \hbar^{-\alpha}$, where $\alpha \sim 1$ (rather than on the much shorter $t_{\hbar} \sim \ln \hbar^{-1}$ we have discussed in Section III) so it can be ignored for macroscopic systems. On the other hand, its signature is easy to detect.

Demonstration of dynamical Anderson localization in the optical lattice implementation of the $\delta$-kicked rotor and related studies of quantum chaos are a significant success (Moore et al., 1994). More recently, attention of both Raizen and his group in Texas as well as of Christensen and his group in New Zealand has shifted towards the effect of decoherence on quantum chaotic evolution (Ammann et al, 1998; Klappauf et al., 1998).

In all of the above studies the state of the chaotic system ( $\delta$-kicked rotor) was perturbed by spontaneous emission from the trapped atoms, that was induced by decreasing detuning of the lasers used to set up the optical lattice. In addition, noise was occasionally introduced into the potential. Both groups find that, as a result of spontaneous emission, localization disappears, although the two studies differ in some of the details. More experiments, including some that allow gentler forms of monitoring by the environment (rather than spontaneous emission noise) appear to be within reach.

In all of the above cases one deals, in effect, with a large ensemble of identical atoms. While each atom suffers repeated disruptions of its evolution due to spontaneous emission, the ensemble evolves smoothly and in accord with the appropriate master equation. The situation is reminiscent of the "decoherence simulated by noise".

Experiments that probe the effect of classical noise on chaotic systems have been carried out earlier (Koch, 1995). They were, however, analyzed from the point of view that does not readily shed light on decoherence.

A novel experimental approach to decoherence and to irreversibility in open complex quantum systems is pursued by Levstein, Pastawski, and their colleagues (Levstein, Usaj, and Pastawski, 1998; Levstein et al., 2000). Using NMR techniques the investigate reversibility of dynamics by implementing a version of spin echo. This promising "Loschmidt echo" approach has led to renewed interest in the issues that touch on quantum chaos, decoherence, and related subjects (see e.g., Gorin and Seligman, 2001; Prosen, 2001; Jalabert and Pastawski, 2001; Jacquod, Silvestrov and Beenakker, 2001).

\section{Analogue of decoherence in a classical system}

Both the system and the environment are effectively classical in the last category of experiments, represented by the work of Cheng and Raymer (1999). They have investigated behavior of transverse spatial coherence during the propagation of the optical beam through a dense, random dielectric medium. This problem can be modelled by a Boltzmann-like transport equation for the Wigner function of the wave field, and exhibits characteristic increase of decoherence rate with the square of the spatial separation, followed by a saturation at sufficiently large distances. This saturation contrasts with the simple models of decoherence in quantum Brownian motion that are based on dipole approximation. However, it is in good accord with the more sophisticated discussions which recognize that, for separations of the order of the prevalent wavelength in the environment, dipole approximation fails and other more complicated 
behaviors can set in (Gallis and Fleming, 1990; Anglin, Paz and Zurek, 1997; Paz and Zurek, 1999). Similar result in a completely quantum case was obtained using atomic interferometry by Kokorowski et al. (2001).

\section{Taming decoherence}

In many of the applications of quantum mechanics the quantum nature of the information stored or processed needs to be protected. Thus, decoherence is an enemy. Quantum computation is an example of this situation. Quantum computer can be thought of as a sophisticated interference device that works by performing in parallel a coherent superposition of a multitude of classical computations. Loss of coherence would disrupt this quantum parallelism essential for the expected speedup.

In the absence of the ideal - completely isolated absolutely perfect quantum computer, something easy for a theorist to imagine but impossible to attain in the laboratory - one must deal with imperfect hardware "leaking" some of its information to the environment. And maintaining isolation while simultaneously achieving a reasonable "clock time" for the quantum computer is likely to be difficult since both are in general controlled by the same interaction (although there are exceptions - for example, in the ion trap proposal of Cirac and Zoller (1995) interaction is in a sense "on demand", and is turned on by the laser coupling internal states of ions with the vibrational degree of freedom of the ion chain).

The need for error correction in quantum computation was realized early on (Zurek, 1984b) but methods for accomplishing this goal have evolved dramatically from the Zeno effect suggested then to very sophisticated (and much more effective) strategies in the recent years. This is fortunate: Without error correction even fairly modest quantum computations (such as factoring number 15 in an ion trap with imperfect control of the duration of laser pulses) go astray rapidly as a consequence of relatively small imperfections (Miquel, Paz, and Zurek, 1997).

Three different, somewhat overlapping approaches that aim to control and tame decoherence, or to correct errors caused by decoherence or by the other imperfections of the hardware have been proposed. We summarize them very briefly, spelling out main ideas and pointing out references that discuss them in greater detail.

\section{Pointer states and noiseless subsystems}

The most straightforward strategy to suppress decoherence is to isolate the system of interest (e.g., quantum computer). Failing that, one may try to isolate some of its observables with degenerate pointer subspaces, which then constitute niches in the Hilbert space of the information processing system that do not get disrupted in spite of its the coupling to the environment. Decoherence free subspaces (DFS's for brevity) are thus identical in conception with the pointer subspaces introduced some time ago (Zurek, 1982), and satisfy (exactly or approximately) the same Eqs. (4.22) and (4.41) or their equivalents (given, e.g., in terms of "Krauss operators" (Krauss, 1983)) that represent non-unitary consequences of the interaction with the environment in the Lindblad (1976) form of the master equation). DFS's were (re)discovered in the context of quantum information processing. They appear as a consequence of an exact or approximate symmetries of the Hamiltonians that govern the evolution of the system and its interaction with the environment (Zanardi and Rasetti, 1997; Duan and Guo, 1998; Lidar et al., 1998; Zanardi, 1998; 2000).

An active extension of this approach aimed at finding quiet corners of the Hilbert space is known as dynamical decoupling. There the effectively decoupled subspaces are induced by time-dependent modifications of the evolution of the system deliberately introduced from the outside by time-dependent evolution and / or measurements (see e.g. Viola and Lloyd, 1998; Zanardi, 2000). A further generalization and unification of various techniques leads to the concept of noiseless quantum subsystems (Knill, Laflamme, and Viola, 1999; Zanardi, 2000), which may be regarded as a non-abelian (and quite non-trivial) generalization of pointer subspaces.

A sophisticated and elegant strategy that can be regarded as a version of the decoherence free approach was devised independently by Kitaev (1997a, b). He has advocated using states that are topologically stable, and, thus, that can successfully resist arbitrary interactions with the environment. The focus here (in contrast to much of the DFS work) is on devising a system with selfHamiltonian that - as a consequence of the structure of the gap its energy spectrum related to the "cost" of topologically non-trivial excitations - acquires a subspace de facto isolated from the environment. This approach has been further developed by Bravyi and Kitaev (1998) and by Freedman and Meyer (1998).

\section{Environment engineering}

This strategy involves altering the (effective) interaction Hamiltonian between the system and the environment or influencing the state of the environment to selectively suppress decoherence. There are many ways to implement it, and we shall describe under this label variety of proposed techniques (some of which are not all that different from the strategies we have just discussed) that aim to protect quantum information stored in selected subspaces of the Hilbert space of the system, or even to exploit pointer states induced or redefined in this fashion.

The basic question that started this line of research - can one influence the choice of the preferred pointer states? - arose in the context of ion trap quantum computer proposed by Cirac and Zoller (1995). The answer given by the theory is, of course, that the choice of the einselected basis is predicated on the details of the situa- 
tion, and, in particular, on the nature of the interaction between the system and the environment (Zurek, 1981; 1982; 1993a). Yet, Poyatos, Cirac and Zoller (1996) have suggested a scheme suitable for implementation in an ion trap, where interaction with the environment - and, in accord with Eq. (4.41), the pointer basis itself - can be adjusted. The key idea is to recognize that the effective coupling between the vibrational degrees of freedom of an ion (the system) and the laser light (which plays the role of the environment) is given by:

$$
H_{\text {int }}=\frac{\Omega}{2}\left(\sigma_{+} e^{-i \omega_{L} t}+\sigma_{-} e^{i \omega_{L} t}\right) \sin \left[\kappa\left(a+a^{+}\right)+\phi\right] .
$$

Above, $\Omega$ is the Rabi frequency, $\omega_{L}$ the laser frequency, $\phi$ is related to the relative position of the center of the trap with respect to the laser standing wave, $\kappa$ is the LambDicke parameter of the transition, while $\sigma_{-}\left(\sigma_{+}\right)$and $a\left(a^{+}\right)$are the annihilation (creation) operators of the atomic transition and of the harmonic oscillator (trap).

By adjusting $\phi$ and $\omega_{L}$ and adopting appropriate set of approximations (that include elimination of the internal degrees of freedom of the atom) one is led to the master equation for the system - i.e, for the density matrix of the vibrational degree of freedom:

$$
\dot{\rho}=\gamma\left(2 f \rho f^{+}-f_{+} f \rho-\rho f_{+} f\right)
$$

Above, $f$ is the operator with a form that depends on the adjustable parameters $\phi$ and $\omega_{L}$ in $H_{\text {int }}$, while $\gamma$ is a constant that depends also on $\Omega$ and $\eta$. As Poyatos et al. show, one can alter the effective interaction between the slow degree of freedom (the oscillator) and the environment (laser light) by adjusting parameters of the actual $H_{\text {int }}$.

First steps towards realization of this "environment engineering" proposals were taken by the NIST group (Myatt et al., 2000; Turchette et al., 2000). Similar techniques can be employed to protect deliberately selected states from decoherence (Carvalho et al., 2000).

Other ideas aimed at channeling decoherence have been also explored in contexts that range from quantum information processing (Beige et al., 2000) to preservation of Schrödinger cats in Bose-Einstein condensates (Dalvit, Dziarmaga, and Zurek, 2000).

\section{Error correction and resilient computing}

This strategy is perhaps most sophisticated and comprehensive, and capable of dealing with the greatest variety of errors in a most hardware - independent manner. It is a direct descendant of the error correction techniques employed in dealing with the classical information, based on redundancy: Multiple copies of the information are made, and the errors are found and corrected by sophisticated "majority voting' techniques.

One might have thought that implementing error correction in the quantum setting will be difficult for two reasons. To begin with, quantum states - and, hence, quantum information - cannot be "cloned" (Dieks, 1982; Wootters and Zurek, 1982). Moreover, quantum information is very "private", and a measurement that is involved in majority voting would infringe on this privacy and destroy quantum coherence, making quantum information classical. Fortunately, both of these difficulties can be simultaneously overcome by encoding quantum information in entangled states of several qubits. Cloning turns out not to be necessary. And measurements can be carried out in a way that identifies errors while keeping quantum information untouched. Moreover, error correction is discrete - measurements that reveal error syndromes have "yes - no" outcomes. Thus, even though information stored in a qubit represents a continuum of possible quantum states (e.g., corresponding to a surface of the Bloch sphere) error correction is discrete, allaying one of the earliest worries concerning the feasibility of quantum computation - the unchecked "drift" of the quantum state representing information (Landauer, 1995).

This strategy (discovered by Shor (1995) and Steane (1996)) has been since investigated by many (Ekert and Macchiavello, 1996; Bennett et al., 1996; Laflamme et al., 1996) and codified into a mathematically appealing formalism (Gottesman, 1996; Knill and Laflamme, 1997). Moreover, first examples of successful implementation (see e.g. Cory et al., 1999) are already at hand.

Error correction allows one, at least in principle, to compute forever, providing that the errors are suitably small $\left(\sim 10^{-4}\right.$ per computational step seems to be the error probability threshold sufficient for most error correction schemes). Strategies that accomplish this encode qubits in already encoded qubits (Aharonov and Ben-Or 1997; Kitaev 1997c; Knill, Laflamme and Zurek, 1996; 1998a, b; Preskill 1998). The number of layers of such concatenation necessary to achieve fault tolerance - the ability to carry out arbitrarily long computations - depends on the size (and the character) of the errors, and on the duration of the computation, but when error probability is smaller than the threshold, that number of layers is finite. Overviews of fault tolerant computation are already at hand (Preskill, 1999; Nielsen and Chuang, 2000, and references therein).

An interesting subject related to the above discussion is quantum process tomography, anticipated by Jones (1994), and described in the context of quantum information processing by Chuang and Nielsen (1997) and by Poyatos, Cirac and Zoller (1997). The aim here is to characterize completely a process - such as a quantum logical gate - and not just a state. First deliberate implementation of this procedure (Nielsen, Knill, and Laflamme, 1998) has also demonstrated experimentally that einselection is indeed equivalent to an unread measurement of the pointer basis by the environment, and can be regarded as such from the standpoint of applications (i.e., NMR teleportation in the example above). 


\section{CONCLUDING REMARKS}

Decoherence, einselection, pointer states, and even predictability sieve have become familiar concepts to many in the past decade. The first goal of this paper was to review these advances and to survey, and - where possible, to address - the remaining difficulties. The second related aim was to "preview" the future developments. This has led to considerations involving information, as well as to the operational, physically motivated discussions of seemingly esoteric concepts such as objectivity. Some of the material presented (including the 'Darwinian' view of the emergence of objectivity through redundancy, as well as the discussion of envariance and probabilities) are rather new, and a subject of research, hence the word "preview" applies here.

New paradigms often take a long time to gain ground. Atomic theory of matter (which, until early XX century, was 'just an interpretation') is the case in point. Some of the most tangible applications and consequences of new ideas are difficult to recognize immediately. In the case of atomic theory, Brownian motion is a good example: Even when the evidence is out there, it is often difficult to decode its significance.

Decoherence and einselection are no exception. They have been investigated for about two decades. They are the only explanation of classicality that does not require modifications of quantum theory, as do the alternatives (Bohm, 1952; Leggett, 1980, 1988, 2002; Penrose, 1986, 1989; Holland, 1993; Goldstein, 1998; Pearle, 1976; 1993; Ghirardi, Rimini, and Weber, 1986; 1987, Gisin and Percival, $1992 ; 1993 \mathrm{a}-\mathrm{c})$. Ideas based on the immersion of the system in the environment have recently gained enough support to be described (by sceptics!) as "the new orthodoxy" (Bub, 1997). This is a dangerous characterization, as it suggests that the interpretation based on the recognition of the role of the environment is both complete and widely accepted. Neither is certainly the case.

Many conceptual and technical issues (such as what constitutes 'a system') are still open. As for the breadth of acceptance, "the new orthodoxy" seems to be an optimistic (mis-)characterization of decoherence and einselection, especially since this explanation of the transition from quantum to classical has (with very few exceptions) not made it to the textbooks. This is intriguing, and may be as much a comment on the way in which quantum physics has been taught, especially on the undergraduate level, as on the status of the theory we have reviewed and its level of acceptance among the physicists.

Quantum mechanics has been to date, by and large, presented in a manner that reflects its historical development. That is, Bohr's planetary model of atom is still often the point of departure, Hamilton-Jacobi equations are used to "derive" Schrödinger equation, and an oversimplified version of the quantum - classical relationship (attributed to Bohr, but generally not doing justice to his much more sophisticated views) with the correspondence principle, kinship of commutators and Poisson brackets,
Ehrenfest theorem, some version of the Copenhagen interpretation, and other evidence that quantum theory is really not all that different from classical - especially when systems of interest become macroscopic, and all one cares about are averages - is presented.

The message seems to be that the there is really no problem and that quantum mechanics can be "tamed" and confined to the microscopic domain. Indeterminacy and double slit experiment are of course discussed, but to prove peaceful coexistence within the elbow room assured by the Heisenberg's principle and complementarity. Entanglement is rarely explored. This is quite consistent with the aim of the introductory quantum mechanics courses, which has been (only slightly unfairly) summed up by the memorable phrase "shut up and calculate". Discussion of measurement is either dealt with through models based on the CI "old orthodoxy", or not at all. An implicit (and sometime explicit) message is: Those who ask questions that do not lend themselves to an answer through laborious, preferably perturbative calculation are "philosophers", and should be avoided.

The above description is of course a caricature. But given that the calculational techniques of quantum theory needed in atomic, nuclear, particle, or condensed matter physics are indeed difficult to master, and given that - to date - most of the applications had nothing to do with the nature of quantum states, entanglement, and such, the attitude of avoiding the most flagrantly quantum aspects of quantum theory is easy to understand.

Novel applications force one to consider questions about the information content, the nature of "the quantum", and the emergence of the classical much more directly, with a focus on states and correlations, rather than on the spectra, cross sections and the expectation values. Hence, problems that are usually bypassed will come to the fore: It is hard to brand Schrödinger cats and entanglement as "exotic" and make them a centerpiece of a marketable device. I believe that as a result decoherence will become a part of the textbook lore. Indeed, at the graduate level there are already some notable exceptions among monographs (Peres, 1993) and specialized texts (Walls and Milburn, 1994; Nielsen and Chuang, 2000).

Moreover, the range of subjects already influenced by decoherence and einselection - by the ideas originally motivated by the quantum theory of measurements - is beginning to extend way beyond its original domain. In addition to the atomic physics, quantum optics, and quantum information processing (which were all mentioned throughout this review) it stretches from material sciences (Karlsson, 1998; Dreismann, 2000), surface science, where it seems to be an essential ingredient explaining emission of electrons (Brodie, 1995; Durakiewicz et al., 2001) through heavy ion collisions (Krzywicki, 1993) to quantum gravity and cosmology (Zeh, 1986, 1988, 1992; Kiefer, 1987; Kiefer and Zeh, 1995; Halliwell, 1989; Brandenberger, Laflamme and Mijic, 1990; Barvinsky and Kamenshchik, 1990, 1995; Paz and Sinha, 1991, 1992; Castagnino et al., 1993, Mensky and Novikov, 1996). 
Given the limitations of space we have not done justice to most of these subjects, focusing instead on issues of principle. In some areas reviews already exist. Thus, Giulini et al. (1996) is a valuable collection of essays, where, for example, decoherence in field theories is addressed. Dissertation of Wallace (2002) offers a good (if somewhat philosophical) summary of the role of decoherence with a rathe different emphasis on similar fieldtheoretic issues. Conference proceedings edited by Blanchard et al. (2000) and, especially, an extensive historical overview of the foundation of quantum theory from the modern perspective by Auletta (2000) are also recommended. More specific technical issues with implications for decoherence and einselection have also been reviewed. For example, on the subject of master equations there are several reviews with very different emphasis including Alicki and Lendi (1987), Grabert, Schramm, and Ingold (1988), Namiki and Pascazio (1993), as well as - more recently - Paz and Zurek (2001). In some areas - such as atomic BEC's - the study of decoherence has only started (Anglin, 1997; Dalvit, Dziarmaga, and Zurek, 2001). In many situations (e.g, quantum optics) a useful supplement to the decoherence view of the quantum - classical interface is afforded by 'quantum trajectories' - a study of the state of the system inferred from the intercepted state of the environment (see Carmichael, 1993; Wiseman and Milburn, 1993; Gisin and Percival, 1993a-c). This approach "unravels" evolving density matrices of open systems into trajectories conditioned upon the measurement carried out on the environment, and may have - especially in quantum optics - intriguing connections with the "environment as a witness" point of view (see Dalvit, Dziarmaga, and Zurek, 2001). In other areas such condensed matter - decoherence phenomena have so many variations and are so pervasive that a separate "decoherent review" may be in order, especially as intriguing experimental puzzles seem to challenge the theory (Mohanty and Webb, 1997; Kravtsov and Altshuler, 1999).

Physics of information and computation is a special case. Decoherence is obviously a key obstacle in the implementation of information processing hardware that takes advantage of the superposition principle. While we have not focused on quantum information processing, the discussion was often couched in the language inspired by the information theory. This is no accident: It is the belief of this author that many of the remaining gaps in our understanding of quantum physics and its relation to the classical domain - such as the definition of systems, or the still mysterious details of the "collapse" - shall follow pattern of the "predictability sieve" and shall be expanded into new areas investigation by considerations that simultaneously elucidate nature of "the quantum" and of "the information".

\section{ACKNOWLEDGMENTS}

John Archibald Wheeler has - quarter century ago - taught a course on the subject of quantum measurements at the University of Texas in Austin. The questions raised then have since evolved into ideas presented here, partly in collaboration with Juan Pablo $\mathrm{Paz}$, and through interactions with many colleagues, including Andreas Albrecht, Jim Anglin, Charles Bennett, Robin Blume-Kohout, Carlton Caves, Ike Chuang, Diego Dalvit, David Deutsch, David Divincenzo, Jacek Dziarmaga, Richard Feynman, Murray Gell-Mann, Daniel Gottesmann, Robert Griffiths, Salman Habib, Jonathan Halliwell, Serge Haroche, James Hartle, Chris Jarzynski, Erich Joos, Manny Knill, Raymond Laflamme, Anthony Leggett, Seth Lloyd, Gerard Milburn, Michael Nielsen, Harold Ollivier, Asher Peres, David Poulin, Rüdiger Schack, Ben Schumacher, Kosuke Shizume, Bill Unruh, David Wallace, Eugene Wigner, Bill Wootters, Dieter Zeh, Anton Zeilenger, and Peter Zoller. Moreover, Serge Haroche, Mike Nielsen, Harold Ollivier, Juan Pablo Paz and David Wallace have provoided me with extensive written comments on earlier versions of the manuscript. Its preparation was assisted by authors' participation in two ITP programs on decoherence - related subjects, and was in part supported by a grant from the NSA. Last not least, this paper has evolved in course of over a dozen years, along with the field, under a watchful eye of a sequence of increasingly impatient editors of RMP, their feelings shared by my family. Perseverance of all afflicted was very much appreciated by the author (if thoroughly tested by the process).

\section{References}

[4] Adler, S. L., 2001, quant-ph/0112095

[4] Aharonov, D, and M. Ben-Or, in Proceedings of the 29th Annual ACM Symposium on the Theory of Computing, pp. 176-188.

[4] Aharonov, Y, J. Anandan, and L. Vaidman, 1993, Phys. Rev. A47, 4616.

[4] Aharonov, Y, B. Resnik, 2002, Phys. Rev. A65, 052116.

[332] Albrecht, A., 1992, Phys. Rev. D46, 5504-5520.

[332] Albrecht, A., 1993, Phys. Rev. D48, 3768-3778.

[332] Alicki R., and K. Lendi, 1987, Quantum Dynamical Semigroups and Applications, \# 286 of Lecture Nores In Physics (Springer, Berlin).

[332] Anderson, P. W., 2001, Stud. Hist. Phil. Mod. Phys. 32, 487-494.

[332] Ambegaokar, V., 1991, Ber. Bunsenges. Phys. Chem. 95, 400-404.

[332] Ammann, H., R. Gray, I. Shvarchuk, and N. Christensen, 1998, Phys. Rev. Lett. 80, 4111.

[332] Anglin, J. R., 1997, Phys. Rev. Lett. 79, 6

[332] Anglin, J. R., and Zurek, W. H., 1996, Phys. Rev. D53, $7327-7335$.

[332] Anglin, J. R., J. P. Paz, and W. H. Zurek, 1997, Phys. Rev. A 53, 4041. 
[332] Auletta, G., 2000, Foundations and Interpretation of Quantum Theory (World Scientific, Singapore).

[332] Bacciagaluppi, G., and M. Hemmo, 1998, pp. 95-114 in Healey and Hellman, 1998

[332] Ballentine, L. E., 1970, Rev. Mod. Phys. 42, 358.

[332] Ballentine, L. E., Y. Yang, and J. P. Zibin, 1994, Phys. Rev. A 50, 2854

[332] Barnett, S. M., and Phoenix, S. J. D., 1989, Phys. Rev. A40, 2404-2409.

[332] Barnum, H., C. M. Caves, J. Finkelstein, C. A. Fuchs, and R. Schack, 2000, Proc. Roy. Soc. London A 456, 1175-1182.

[332] Barvinsky, A. O., and A. Yu. Kamenshchik, 1990, Class. Quant. Grav., 7, 2285-2293.

[332] Barvinsky, A. O., and A. Yu. Kamenshchik, 1995, Phys. Rev. D 52 743-757.

[332] Beckman, D., D. Gottesman, and M. A. Nielsen, 2001, quant-ph/0102043

[332] Beige, A., D. Braun, D. Tregenna, P. L. Knight, 2000, Phys. Rev. Lett. 85, 1762.

[332] Bell, J. S., 1981, in Quantum Gravity 2, edited by C. Isham, R. Penrose and D. Sciama, (Clarendon Press, Oxford) pp. 611-637.

[332] Bell, J. S., 1987, Speakable and Unspeakable in Quantum Mechanics, (Cambridge University Press).

[332] Bell, J. S., 1990, "Against 'Measurement' ", in SixtyTwo Years of Uncertainty, edited by A. I. Miller, (Plenum, NATO Series, New York).

[332] Bennett, C. H., and D. P. DiVincenzo, 2000, Nature 404, 247.

[332] Bennett, C. H., D. P. DiVincenzo, J. Smolin, and W. K. Wootters, 1996, Phys. Rev. A 54, 3824.

[332] Berman, G. P., and G. M. Zaslavsky, 1978, Physica 91A, 450.

[332] Berry, M. V., and N. L. Balazs, 1979, J. Phys. A 12, 625.

[332] Berry, M. V. 1995, in Fundamental Problems in Quantum Theory, edited by Greenberger, D. M. and Zeilinger, A. (N. Y. Academy of Sciences, New York) pp. 303-317.

[332] Birkhoff, G., and J. von Neumann, 1936, Ann. Math. 37, 823-843

[332] Blanchard, Ph., D. Giulini, E. Joos, C. Kiefer, I.-O. Stamatescu, 2000, Decoherence: Theoretical, Experimental, and Conceptual Problems (Springer, Berlin)

[332] Blanchard Ph., and R. Olkiewicz, 2000, Phys. Lett. A 273223.

[332] Boccaletti, S., A. Farini, and F. T. Arecchi, 1997, Phys. Rev. E 554979.

[332] Bocko, M. F., and R. Onofrio, 1996, Rev. Mod. Phys. 68, 755 .

[332] Bogolubov, N. N., Logunov, A. A., Oksak, A. I., and Todorov, I. T, 1990, General Principles of Quantum Field Theory, Kluwer, Dordrecht.

[332] Bohm, D., 1952, Phys. Rev. 85, 166-193; reprinted in Wheeler and Zurek, 1983.

[332] Bohr, N., 1928, Nature 121, 580-590; [reprinted in Wheeler and Zurek, 1983].

[332] Bohr, N., 1935, Phys. Rev. 48, 696-702; [reprinted in Wheeler and Zurek, 1983].

[332] Bohr, N., 1949, Albert Einstein: Philosopher-Scientist, edited by P. A. Schilpp, (Open Court Publishing, Evanston, pp. 200-241); [reprinted in Wheeler and Zurek, 1983].
[332] Borel, E., 1914, Introduction Géométrique à Quelques Théories Physiques (Gauthier-Villars, Paris).

[332] Born, M., 1969, Albert Einstein, Hedwig und Max Born, Briefwechsel 1916-1955, (Nymphenburger Verlagshandlung, München).

[332] Braginski, V. B., Vorontsov, Y. I., and Thorne, K. S., 1980, Science 209, 547-557; reprinted in Wheeler and Zurek, 1983.

[332] Braginsky, V. B., and F. Ya. Khalili, 1996, Rev. Mod. Phys. 68, 1.

[332] Brandenberger, R., R. Laflamme, and M. Mijic, 1990, Mod. Phys. Lett., A5, 2311.

[332] Braun, D., P. A. Braun, and F. Haake, 2000, Opt. Comm. 179, 411.

[332] Bravyi, S. B., and A. Y. Kitaev, 1998, quant-ph 9811052

[332] Brillouin, L., 1962, Science and Information Theory, (Academic Press, New York).

[332] Brillouin, L., 1964, Scientific Uncertainty, and Information, (Academic Press, New York).

[332] Brodie, I., 1995, Phys. Rev. B 51, 13660-13668.

[332] Brun, T., and J. B. Hartle, 1999, Phys. Rev. D 6012 1165.

[332] Brun, T., I. C. Percival, and R. Schack, 1995, J. Phys. A 29, 2077.

[332] Brune, M., E. Hagley, J. Dreyer, X. Maitre, C. Wunderlich, J.-M. Raimond, and S. Haroche, 1996, Phys. Rev. Lett 77, 4887.

[332] Bruss, D., A. Ekert and C. Macchiavello, 1999, Phys. Rev. Lett. 812598.

[332] Bub, J., 1997, Interpreting the Quantum World, Cambridge University Press, Cambridge.

[332] Butterfield, J., 1996, Brit. J. Phil. Sci. 47, 200-221.

[332] Caldeira, A. O., and Leggett, A. J., 1983, Physica 121A, 587-616.

[332] Carmichael, H. J., 1993, An Open Systems Approach to Quantum Optics (Springer, Berlin).

[332] Carvalho, A. R. R., P. Milman, R. L. de Matos Filho, L. Davidovich, 2000, e-print 0009024

[332] Casati, G., and Chirikov, B. V., 1995a, Quantum Chaos: Between Order and Disorder (Cambridge University Press).

[332] Casati, G., and Chirikov, B. V., 1995b, Phys. Rev. Lett. 75, 2507.

[332] Castagnino, M. A., A. Gangui, F. D. Mazzitelli, and I. I. Tkachev, 1993, Class. Quant. Grav. 10, 2495-2504.

[332] Caves, C. M., Thorne, K. S., Drever, R. W. P., Sandberg, V. P., and Zimmerman, M., 1980, Rev. Mod. Phys. 52, 341-392.

[332] Caves, C. M., 1994, in Physical Origins of Time Asymmetry, edited by J. J. Halliwell, J. Perez-Mercader, and W. H. Zurek (Cambridge University Press, Cambridge), p. 47.

[332] Cerf, N. and Adami, C., 1997, Phys. Rev. A 55, 33713374 .

[332] Chapman, M. S., T. D. Hammond, A. Lenef, J. Schmiedmayer, R. A. Rubenstein, E. Smith, and D. E. Pritchard, 1995, Phys. Rev. Lett. 75, 3783-3787.

[332] Cheng, C. C., and M. G. Raymer, 1999, Phys. Rev. Lett 82, 4802 .

[332] Chuang, I. L., and M. A. Nielsen, J. Mod. Opt. 44, 2455-2467.

[332] Cirac, J. I., and P. Zoller, 1995, Phys. Rev. Lett. 74, 4091 
[332] Cory, D. G., W. Mass, M. Price, E Knill, R. Laflamme, W. H. Zurek, T. F. Havel, and S. S. Somaroo, 1999, Phys. Rev. Lett. 81, 2152-2155.

[332] Cover, T. M, and J. A. Thomas, 1991 Elements of Information Theory (Wiley, New York).

[332] Cox, R. T., 1946, Am. J. Phys. 14, 1.

[332] Dalvit, D., J. Dziarmaga, and W. H. Zurek, 2000, Phys. Rev. A 62, 013607.

[332] Dalvit, D., J. Dziarmaga, and W. H. Zurek, 2001, Phys. Rev. Lett. 86, 373.

[332] Dekker, H., 1977, Phys. Rev. A16, 2126-2134.

[332] d'Espagnat, B., 1976, Conceptual Foundations of Quantum Mechanics, W. A. Benjamin, (Reading, MA).

[332] d'Espagnat, B., 1989, J. Stat. Phys. 58, 747-766.

[332] d'Espagnat, B., 1995, Veiled Reality, Addison-Wesley, Reading, MA. [1, 17, 37, 167, 170, 171, 186]

[332] Deutsch, D., 1985, Int. J. Theor. Phys. 24, 1.

[332] Deutsch, D., 1997, The Fabric of Reality, (Penguin, New York).

[332] Deutsch, D., 1999, Proc. Roy. Soc. London A 4553129.

[332] Deutch, D., 2001 quant-ph/0104033

[332] DeWitt, B. S., 1970, Physics Today, 23, 30-35.

[332] DeWitt, B. S., 1998, pp. 39-49 in Frontiers in Quantum Physics, S. C. Lim, R. Abd-Shukor, and K. H. Kwek, eds. (Springer, Singapore).

[332] DeWitt, B. S., and Graham, N., 1973, The ManyWorlds Interpretation of Quantum Mechanics (Princeton University Press).

[332] Dieks, D., 1982, Phys. Lett. A 92, 271.

[332] Dirac, P. A. M., 1947, The Principles of Quantum Mechanics (Clarendon Press, Oxford)

[332] Donald, M. J., 1995, Found. Phys. 25, 529-571.

[332] Dowker, H. F., and Halliwell, J. J., 1992, Phys. Rev. D46, 1580-1609.

[332] Dowker, H. F., and Kent, A., 1996, J. Stat. Phys. 82, 1575-1646.

[332] Duan, L.-M., and G.-C. Guo, 1998, Phys. Rev. A 57, 737-741.

[332] Durakiewicz, T., S. Halas, A. Arko, J. J. Joyce, and D. P. Moore, 2001, Phys. Rev. B 6404, 5101.

[332] Einstein, A., 1917, Verh. Deutsch. Phys. Ges. 19, 82.

[332] Einstein, A., B. Podolsky, and N. Rosen, 1935, Phys. Rev. 47, 777-780; [reprinted in Wheeler and Zurek, 1983].

[332] Eisert, J., and M. B. Plenio, 2002, Phys. Rev. Lett., in press.

[332] Ekert, A., and C. Macchiavello, 1996, Phys. Rev. Lett. 77, 2585.

[332] Elby, A., 1993, in Symposium on the Foundations of Modern Physics, edited by P. Busch, P. Lahti, and P. Mittelstaedt (World Scientific, Singapore), p. 168.

[332] Elby, A., 1998, pp. 87-94 in Healey and Hellmann, 1998

[332] Elby, A., and Bub, J., 1994, Phys. Rev. A49, 42134216.

[332] Emerson, J., and L. E. Ballentine, 2001a, Phys. Rev. A63, 052103.

[332] Emerson, J., and L. E. Ballentine, 2001b, Phys. Rev. E64, 026217.

[332] Everett, H., III., 1957a, Rev. Mod. Phys. 29, 454-462; reprinted in Wheeler and Zurek, 1983.

[332] Everett, H., III, 1957b, The Theory of the Universal Wave Function, Princeton University Dissertation; reprinted in DeWitt and Graham, 1973.

[332] Farhi, E., J. Goldstone, and S. Guttmann, 1989, Ann.
Phys. 192, 368.

[332] Feynman, R. P., and Vernon, F. L., Jr., 1963, Ann. Phys. (N.Y.) 24, 118-173.

[332] Ford, J., and Mantica, G., 1992, Am. J. Phys. 60, 10861098.

[332] Fox, R. F., and T. C. Elston, 1994, Phys. Rev. E49, 3683; ibid. E50, 2553.

[332] Freedman, M. H., and D. A. Meyer, 1998, quant-ph 9810055

[332] Furuya, K., M. C. Nemes, and G. Q. Pellegrino, 1998, Phys. Rev. Lett. 80, 5524

[332] Fuchs, C. A., and A. Peres, 2000, Phys. Today 53, 70.

[332] Gallis, M. R., 1992, Phys. Rev. A45, 47-53.

[332] Gallis, M. R., 1996, Phys. Rev. A53, 655-660.

[332] Gallis, M. R., and Fleming, G. N., 1990, Phys. Rev. A42, 38-48.

[332] Gell-Mann, M., and Hartle, J. B., 1990, Complexity Entropy, and the Physics of Information, edited by W. H. Zurek, Addison-Wesley, Reading, pp. 425-458.

[332] Gell-Mann, M., and Hartle, J. B., 1993, Phys. Rev. D47, 3345-3382.

[332] Gell-Mann, M., and J. B. Hartle, 1997, in Quantum Classical Correspondence, edited by Feng, D. H., and $\mathrm{Hu}, \mathrm{B}$. L. (International Press, Cambridge, MA), p.3.

[332] Geroch, R., 1984, Noûs, XVIII, 617-634.

[332] Ghirardi, G. C., Rimini, A., and Weber, T., 1986, Phys. Rev. D34, 470-491.

[332] Ghirardi, G. C., Rimini, A., and Weber, T., 1987, Phys. Rev. D36, 3287-3289.

[332] Gisin, N., and Percival, I. C., 1992, J. Phys. A: Math. Gen. 25, 5677-5691.

[332] Gisin, N., and Percival, I. C., 1993a, J. Phys. A: Math. Gen. 26, 2233-2243.

[332] Gisin, N., and Percival, I. C., 1993b, J. Phys. A: Math. Gen. 26, 2245-2260.

[332] Gisin, N., and Percival, I. C., 1993c, Phys. Lett. A175, 144-145.

[332] Giulini, D., 2000, pp. 67-91 in Relativistic Quantum Measurement and Decoherence, H.-P. Breuer and F. Petruccione, eds. (Springer, Berlin).

[332] Giulini, D., Kiefer, C., and Zeh, H. D., 1995, Phys. Lett. A199, 291-298.

[332] Giulini, D., E. Joos, C. Kiefer, J. Kupsch, I.-O. Stamatescu, and H. D. Zeh, 1996, Decoherence and the Appearance of a Classica World in Quantum Theory (Springer, Berlin)

[332] Gleason, A. M., 1957, Journ. Math. Mech. 6, 885-893.

[332] Gnedenko, B. V., 1982, The Theory of Probability, (MIR Publishers, Moscow).

[332] Goldstein, S., 1998, Physics Today, 52, \#3, 42-46 (part I) and \#4, 38-42 (part II).

[332] Goldstein, S., and D. N. Page, 1995, Phys. Rev. Lett. 74, 3715-3719.

[332] Gorin, T., and Seligman, T. H., 2001, quant-ph/0112003).

[332] Gorini, V., A. Kossakowski, and E. C. G. Sudarshan, 1976, J. Math. Phys. 17, 821-825.

[332] Gottesman, D., 1996, Phys. Rev. A 54, 1862.

[332] Gottfried, K., 1966, Quantum Mechanics, section 20 (Benjamin, New York).

[332] Gottfried, K., 2000, Nature 405, 533-536.

[332] Grabert, H., Schramm, P., and Ingold, G.-L., 1988, Phys. Rep. 168, 115-207.

[332] Graham, N., 1970, The Everett Interpretation of Quan- 
tum Mechanics, (University North Carolina, Chapel Hill).

[332] Graham, R., M. Schlautmann, and P. Zoller, 1992, Phys. Rev. A 45 R19-R22.

[332] Griffiths, R. B., 1984, J. Stat. Phys. 36, 219-272.

[332] Griffiths, R. B., 1996, Phys. Rev. A 54, 2759.

[332] Griffiths, R. B, and Omnès, R., 1999, Physics Today, 52, 26-31.

[332] Haake, F., 1991, Quantum Signatures of Chaos, Springer, Berlin.

[332] Haake, F., and R. Reibold, 1985, Phys. Rev. A32, 2462.

[332] Haake, F., M. Kuś, and R. Sharf, 1987, Z. Phys. B65, 381.

[332] Haake, F., and D. F. Walls, 1987, Phys. Rev. A36, 730739.

[332] Habib, S., K. Shizume, and W. H. Zurek, 1998, Phys. Rev. Lett. 80, 4361.

[332] Halliwell, J. J., 1989, Phys. Rev. D 39, 2912-2923

[332] Halliwell, J. J., 1999, Phys. Rev. D 60105031.

[332] Halliwell, J. J., J. Péres-Mercader, and W. H. Zurek, eds., 1994, Physical Origins of Time Asymmetry (Cambridge University Press).

[332] Haroche, S., 1998, Physica Scripta T76, 159.

[332] Hartle, J. B., 1968, Am. J. Phys. 36, 704.

[332] Healey, R. A., 1998, pp. 52-86 in Healey and Hellmann (1998).

[332] Healey, R. A., and G. Hellman, eds., 1998, Quantum Measurement: Beyond Paradox, University of Minnesota Press Minneapolis.

[332] Heisenberg, W., 1927, Z. Phys.43, 172-198; [English translation in Wheeler and Zurek, 1983].

[332] Herzog, T. J., P. G. Kwiat, H. Weinfurter, and A. Zeilinger, 1995, Phys. Rev. Lett. 75 3034-3037

[332] Hillery, M. A., and M. O. Scully, 1983, in Quantum Optics, Experimental Gravitation, and Measurement Theory edited by P. Meystre and M. O. Scully (Plenum, New York), p. 65.

[332] Holland, P. R., 1993, The Quantum Theory of Motion (Cambridge University Press, Cambridge).

[332] Hu, B. L., Paz, J. P., and Zhang, Y., 1992, Phys. Rev. D45, 2843-2861.

[332] Hu, B. L., Paz, J. P., and Zhang, Y., 1993, Phys. Rev. D47, 1576-1594.

[332] Ivanovic, I. D., 1981, J. Phys. A 14, 3241-3245.

[332] Jacquod, P., Silvestrov, P. G., and Beenakker, C. W. J., 2001, Phys. Rev. E 64, 055203 (R).

[332] Jalabert, R. A., and H. M. Pastawski, 2001, Phys. Rev. Lett. 862490.

[332] Jammer, M., 1974, The Philosophy of Quantum Mechanics, (Wiley, New York).

[332] Jaynes, E. T., 1957, Phys. Rev. 106, 620-630.

[332] Jones, K. R. W., 1994, Phys. Rev. A 50, 3682-3699.

[332] Joos, E., 1986a, Ann. New York Acad. Sci. 480, 6-13.

[332] Joos, E., and Zeh, H. D., 1985, Z. Phys. B59, 223-243.

[332] Jozsa, R., 2002, quant-ph/02044153.

[332] Karkuszewski, Z. P., C. Jarzynski, and W. H. Zurek, 2002, Phys. Rev. Lett, submitted.

[332] Karkuszewski, Z. P., J. M. Zakrzewski, and W. H. Zurek, 2002, Phys. Rev. A 65, 042113.

[332] Karlsson, E. B., 1998, Physica Scripta T 76, 179-185.

[332] Kent, A., 1990, Int. J. Mod. Phys., 5, 1745-1762.

[332] Kiefer, C., 1987, Class. Quant. Grav. 4 1369-1382.

[332] Kiefer, C., 1996, in Giulini et al., (1996), p. 157.

[332] Kiefer, C., and H. D. Zeh, 1995, Phys. Rev. D 51, 4145-
4153.

[332] Kitaev, A. Y., 1997a, quant-ph 9707021

[332] Kitaev, A. Y., 1997b, Russ. Math. Surv. 52, 1191-1249.

[332] Kitaev, A. Y., 1997c, in Quantum Communication, Computing, and Measurement, pp. 181-188.

[332] Klappauf, B. G., W. H. Oskay, D. A. Steck, and M. G. Raizen, 1998, Phys. Rev. Lett. 81, 1203; erratum in 1999, Phys. Rev. Lett. 82, 241.

[332] Knill, E., and R. Laflamme, 1997, Phys. Rev. A 55, 900.

[332] Knill, E., R. Laflamme, and L. Viola, 2000, Phys. Rev. Lett. 84, 2525.

[332] Knill, E., R. Laflamme, and W. H. Zurek, 1996, quant-ph/96010011.

[332] Knill, E., R. Laflamme, and W. H. Zurek, 1998a, Science 279, 324-345.

[332] Knill, E., R. Laflamme, and W. H. Zurek, 1998b, Proc. Roy. Soc. London A 454, 365-384.

[332] Koch, P. M., 1995, Physica D 83, 178.

[332] Kokorowski, D., A. D. Cronin, T. D. Roberts, and D. E. Pritchard, 2001, Phys. Rev. Lett., in press.

[332] Kolmogorov, A. N., 1960, Math. Rev. 21, 2035.

[332] Kraus, K., 1983, States, Effects, and Operations (Springer, Berlin).

[332] Kravtsov, V. E., and B. L. Altshuler, 2000, Phys. Rev. Lett., 84, 3394.

[332] Krzywicki, A., 1993, Phys. Rev. D48, 5190.

[332] Kwiat, P. G., A. M. Steinberg, and R. Y. Chiao, 1992, Phys. Rev. A 45, 7729-7739.

[332] Laflamme, R., C. Miquel, J. P. Paz, and W. H. Zurek, 1996, Phys. Rev. Lett. 77, 198.

[332] Landau, L., 1927, Zeits. Phys. 45, 430-441.

[332] Landauer, R., 1991, Physics Today 44, 23.

[332] Landauer, R., 1995, Phil. Trans. Roy. Soc. Lond. 353, 367.

[332] Laskar, J., 1989, Nature 338, 237.

[332] Leggett, A. J., 1980, Supp. Prog. Theor. Phys. 69, 80100 .

[332] Leggett, A. J., 1998, in Quantum Measurement, edited by R. A. Healey and G. Hellman (University of Minnesota Press, Minneapolis), p.1.

[332] Leggett, A. J., 2002, Physica Scripta, in press.

[332] Levstein, P. R., G. Usaj, H. M. Pastawski, J. Raya, and J. Hirschinger, 2000, J. Chem Phy. 113, 6285.

[332] Levstein, P. R., G. Usaj, and H. M. Pastawski, 1998, J. Chem. Phys. 108, 2718.

[332] Lidar, D. A., D. Bacon, and K. B. Whaley, 1999, Phys. Rev. Lett. 82, 4556.

[332] Lieb, E. H., 1975, Bull. AMS 81, 1.

[332] Lindblad, G., 1976, Comm. Math. Phys. 48, 119.

[332] Lloyd, S., 1997, Phys. Rev. A55, 1613.

[332] London, F., and Bauer, E., 1939, La Théorie de l'Observation en Méchanique Quantique (Hermann, Paris); English translation in Wheeler and Zurek, 1983.

[332] Mensky, M. B., and I. D. Novikov, 1996, Int. J. Mod. Phys. D5, 1-27.

[332] Mermin, N. D., 1990a, Physics Today 43, 9-11.

[332] Mermin, N. D., 1990b, Am. J. Phys. 58, 731-734.

[332] Mermin, N. D., 1994, Physics Today 47, 9-11.

[332] Miquel, C., J. P. Paz, and W. H. Zurek, 1997, Phys. Rev. Lett.

[332] Miller, P. A., S. Sarkar, and R. Zarum, 1998, Acta Phys. Pol. B29, 4643.

[332] Miller, P. A., and S. Sarkar, 1999, Phys. Rev. E 60, 1542 . 
[332] Mohanty, P., and R. A. Webb, 1997, Phys. Rev. B 55, R13 452

[332] Monroe, C., Meekhof, D. M., King, B. E., and Wineland, D. J., 1996, A 'Schrödinger Cat' superposition state of an atom, Science 272, 1131-1136.

[332] Monteoliva, D., and J.-P. Paz, 2000, Phys. Rev. Lett. 85, 3373.

[332] Moore F. L. et al, 1994, Phys. Rev. Lett. 73, 2974.

[332] Mott, N. F, 1929, Proc. Roy. Soc. London A 126, 79-84; reprinted in Wheeler and Zurek, 1983.

[332] Moyal, J. E., 1949, Proc. Camb. Phil. Soc. 45, 99.

[332] Myatt, C. J., et al., 2000, Nature 403, 269.

[332] Namiki, M., and S. Pascazio, 1993, Phys. Rep. 232, 301.

[332] Nielsen, M. A., and I. L. Chuang, 2000, Quantum Computation and Quantum Information (Cambridge University Press, Cambridge)

[332] Nielsen, M. A., E. Knill, and R. Laflamme, 1998, Nature 396, $52-55$.

[332] Ollivier, H., D. Poulin, and W. H. Zurek, 2002, in preparation.

[332] Ollivier, H., and W. H. Zurek, 2002, Phys. Rev. Lett. 88, 017901.

[332] Omnès, R., 1988, J. Stat. Phys. 53, 893-975.

[332] Omnès, R., 1992, Rev. Mod. Phys. 64, 339-382.

[332] Omnès, R., 1994, The Interpretation of Quantum Mechanics (Princeton University Press, Princeton).

[332] Paraoanu, G.-S. 2002, quant-ph 0205127

[332] Pattanyak, A. K., 2000, Phys. Rev. Lett. 83, 4526

[332] Paz, J.-P., 1994, in Physical Origins of Time Asymmetry, pp. 213-220.

[332] Paz, J. P., Habib, S., and Zurek, W. H., 1993, Phys. Rev. D47, 488-501.

[332] Paz, J.-P., and S. Sinha, 1991, Phys. Rev. D 44, 1038.

[332] Paz, J.-P., and S. Sinha, 1992, Phys. Rev. D 45, 2823.

[332] Paz, J.-P., and W. H. Zurek, 1999, Phys. Rev. Lett. 82, 5181.

[332] Paz, J.-P., and W. H. Zurek, 2001, pp. 533-614 in Coherent Atomic Matter Waves, Les Houches Lectures, R. Kaiser, C. Westbrook, and F. David, eds. (Springer, Berlin).

[332] Pearle, P., 1976, Phys. Rev. D13, 857-868.

[332] Pearle, P., 1993, Phys. Rev. A48, 913-923.

[332] Penrose, R., 1986, Quantum Concepts in Space and Time, edited by C. J. Isham and R. Penrose, (Clarendon Press) pp. 129-146.

[332] Penrose, R., 1989, The Emperors New Mind, (Oxford University Press, Oxford).

[332] Perelomov, 1986, Generalized Coherent States and Their Applications (Springer, Berlin)

[332] Peres, A., 1993, Quantum Theory: Concepts and Methods (Kluwer, Dordrecht).

[332] Pfau, T., Spälter, S., Kurtsiefer, C., Ekstrom, C. R., and Mlynek, J., 1994, Phys. Rev. Lett. 73, 1223-1226.

[332] Poyatos, J. F., Cirac, J. I., and Zoller, P., 1996, Phys. Rev. Lett. 77, 4728.

[332] Poyatos, J. F., Cirac, J. I., and Zoller, P., 1997, Phys. Rev. Lett. 78, 390-393.

[332] Preskill, J., 1998, Proc. Roy. Soc. London A 454, 385410.

[332] Preskill, J., 1999, Phys. Today 52 (\#6) 24-30.

[332] Prosen, T., 2001, quant-ph/0106149

[332] Raimond, J. M., M. Brune, and S. Haroche, 2001, Rev. Mod. Phys. 73, 565-582.

[332] Rauch, H., 1998, Physica Scripta T76, 24.
[332] Reichl, L. E., 1992, Transition to Chaos in Conservative Classical Systems: Quantum Manifestations (Springer, Berlin).

[332] Romero, L. D., and J. P. Paz, 1997, Phys. Rev. A53, 4070.

[332] Saunders, S., 1998, Synthese 114, 373-404.

[332] Schack, R., 1998, Phys. Rev. A 57, 1634

[332] Schack, R. and C. M., Caves, 1996, Phys. Rev. E 53, 3387

[332] Schrödinger, E., 1926, Naturwiss 14, 664-666.

[332] Schrödinger, E., 1935a, Nautrwiss 23, 807-812, 823828, 844-849; [English translation in Wheeler and Zurek, 1983].

[332] Schrödinger, E., 1935b, Proc. Cambridge Phil. Soc. 31, $555-563$.

[332] Schrödinger, E., 1936, Proc. Cambridge Phil. Soc. bf 32, 446-452.

[332] Schumacher, B. W., 1996, Phys. Rev. A54, 2614-2628.

[332] Schumacher, B. W., and M. A. Nielsen, 1996, Phys. Rev. A54, 2629.

[332] Schumacher, B. W., 1999, private communication concerning distinguishability sieve.

[332] Shiokawa, K., and Hu, B. L., 1995, Phys. Rev. E52, 2497-2509.

[332] Shor, P. W., 1995, Phys. Rev. A 522493

[332] Sinai, Ya., 1960, Math. Rev. 212036.

[332] Squires, E. J., 1990, Phys. Lett. A 145, 67-68

[332] Stapp, H. P., 2001, Can. J. Phys, to appear, quant-ph/0110148

[332] Steane, A. M., 1996, Proc. Roy. Soc. London A 452, 2551-2576.

[332] Stein, H., 1984, Noûs XVIII, 635-652.

[332] Stern, A., Y. Aharonow, and Y. Imry, 1989, Phys. Rev. A 413436.

[332] Sussman, G. J., and J. Wisdom, 1992, Science 257, 56.

[332] Tegmark, M., 2000, Phys. Rev. E61, 4194.

[332] Tegmark, M., and Shapiro, H. S., 1994, Phys. Rev. E50, $2538-2547$.

[332] Tegmark, M., and Wheeler, J. A., 2001, Sci. American, 284, 68-75.

[332] Turchette Q. A., et al.,, 2000, Phys. Rev. A 62053807.

[332] Unruh, W. G., 1994, Phys. Rev. A50, 882.

[332] Unruh, W. G., and Zurek, W. H., 1989, Phys. Rev. D40, 1071-1094.

[332] Venugopalan, A., 1994, Phys. Rev. A50, 2742-2745.

[332] Venugopalan, A., 2000, Phys. Rev. A6001, 2102.

[332] Viola, L., and S. Lloyd, 1998, Phys. Rev. A58, 2733.

[332] von Neumann, J., 1932, Mathematische Grundlagen der Quantenmechanik, (Springer, Berlin); reprinted 1981; English translation by R. T. Beyer, 1955: Mathematical Foundations of Quantum Mechanics, (Princeton University Press).

[332] Wallace, D., 2002, Issues in the Foundations of Relativistic Quantum Theory, Merton College, University of Oxford.

[332] Walls, D. F., and Milburn, G. J., 1994, Quantum Optics, Springer, Berlin.

[332] Wehrl, A., 1978, General properties of entropy, Rev. Mod. Phys. 50, 221-260.

[332] Wheeler, J. A., 1957, Rev. Mod. Phys. 29, 463-465.

[332] Wheeler, J. A., 1978, Mathematical Foundations of Quantum Theory, edited by A. R. Marlow, (Academic Press, New York), pp. 9-48.

[332] Wheeler, J. A., 1983, in Wheeler and Zurek, 1983. 
[332] Wheeler, J. A., and W. H. Zurek, 1983, Quantum Theory and Measurement, (Princeton University Press).

[332] Wick, G. C., Wightman, A. S., and Wigner, E. P., 1952, Phys. Rev. 88, 101-105.

[332] Wick, G. C., Wightman, A. S., and Wigner, E. P., 1970, Phys. Rev. D1, 3267-3269.

[332] Wightman, A. S., 1995, Il Nuovo Cimento 110B, 751769.

[332] Wigner, E. P., 1963, Am. J. Phys. 31, 6-15; reprinted in Wheeler and Zurek, 1983.

[332] Wisdom, J., 1985, Icarus, 63272.

[332] Wiseman, H. M., and G. J. Milburn, 1993, Phys. Rev. Lett. 70, 548.

[332] Wiseman, H. M., and J. A. Vaccaro, 1998, Phys. Lett. A 250241.

[332] Wootters, W. K. and Fields, B. D., 1989, Ann. Phys. (N. Y.) 191, 363-381.

[332] Wootters, W. K. and W. H. Zurek, 1979, Phys. Rev. D 19, 473-484

[332] Wootters, W. K. and W. H. Zurek, 1982, Nature 299, 802.

[332] Zanardi, P., 1998, Phys. Rev. A 573276.

[332] Zanardi, P., 2000, Phys. Rev. A, 63 40304, e-print quant-ph9910016

[332] Zanardi, P., and M. Rasetti, 1997, Phys. Rev. Lett. 79, 3306. 79, 3306-3309.

[332] Zeh, H. D., 1970, Found. Phys. 1, 69-76; reprinted in Wheeler and Zurek, 1983.

[332] Zeh, H. D., 1971, in Foundations of Quantum Mechanics, edited by B. d'Espagnat, (Academic Press, New York) pp. 263-273.

[332] Zeh, H. D., 1973, Found. Phys. 3, 109-116.

[332] Zeh, H. D., 1986, Phys. Lett. A 116, 9-12.

[332] Zeh, H. D., 1988, Phys. Lett. A 172, 311-317.

[332] Zeh, H. D., 1990, in Complexity, Entropy, and the Physics of Information, edited by W. H. Zurek, (Addison-Wesley, Redwood City) p. 405.

[332] Zeh, H. D., 1992, The Physical basis of the Direction of Time (Springer, Berlin)

[332] Zeh, H. D., 1993, Phys. Lett. A172, 189-192.

[332] Zeh, H. D., 1997, in New Developments on Fundamental Problems in Quantum Physics, M. Ferrero and A. van der Merwe, eds. (Kluwer, Dordrecht).

[332] Zeh, H. D., 2000, in Decoherence: Theoretical, Experimental, and Conceptual Problems, edited by Ph. Blanchard et al., (Springer, Berlin), p.19.

[332] Zurek, W. H., 1981, Phys. Rev. D24, 1516-1525.

[332] Zurek, W. H., 1982, Phys. Rev. D26, 1862-1880.

[332] Zurek, W. H., 1983, in Quantum Optics, Experimental Gravitation, and Measurement Theory, edited by P. Meystre and M. O. Scully (Plenum, New York), p. 87.

[332] Zurek, W. H., 1984a, Reduction of the wave packet: How long does it take?, Los Alamos Preprint LAUR 84-2750, pp. 145-149 in G. T. Moore and M. T. Scully, eds. Frontiers in Nonequilibrium Statistical Physics (Plenum, New York, 1986)

[332] Zurek, W H., 1984b, Phys. Rev. Lett. 53, 391.

[332] Zurek, W. H., 1989, Phys. Rev. A40, 4731.

[332] Zurek, W. H., 1991, Physics Today 44 (Oct.), 36-44.

[332] Zurek, W. H., 1993a, Progr. Theor. Phys. 89, 281-312.

[332] Zurek, W. H., 1993b, Physics Today (letters) 46 (April), 13.

[332] Zurek, W. H., 1994, Decoherence and the Existential Interpretation of Quantum Theory, or "No Information
Without Representation", pp. 341-350 of From Statistical Physics to Statistical Inference and Back, P. Grassberger and J.-P. Nadal, eds. (Plenum, Dordrecht, 1994).

[332] Zurek, W. H., 1998a, Phil. Trans. Roy. Soc. Lond. A356, 1793.

[332] Zurek, W. H., 1998b, Physica Scripta T76, 186.

[332] Zurek, W. H., 2000, Ann. der Physik, 9, 855.

[332] Zurek, W. H., 2001, Nature, 412, 712-717.

[332] Zurek, W. H., 2002a, Phys. Rev. A, in press.

[332] Zurek, W. H., 2002b, quant-ph/0211037

[332] Zurek, W. H., Habib, S., and Paz, J.-P., 1993, Phys. Rev. Lett. 70, 1187-1190.

[332] Zurek, W. H., and Paz, J.-P., 1994, Phys. Rev. Lett. 72 , 2508-2511.

[332] Zurek, W. H., and Paz, J.-P., 1995a, Physica D83, 300308.

[332] Zurek, W. H., and Paz, J.-P., 1995b, Phys. Rev. Lett. $\mathbf{7 2}, 2508$.

Figure Captions

Fig. 1. Snapshots of the quantum $(\hbar=0.16)$ Wigner function (a-c) and the classical probability distribution in phase space (d) for the chaotic evolution generated from the same initial Gaussian by the Hamiltonian:

$$
H=p^{2} / 2 m-\kappa \cos (x-l \sin t)+a x^{2} / 2
$$

For $m=1, \kappa=0.36, l=3$ and $a=0-0.01$ it exhibits chaos with Lyapunov exponent $\Lambda=0.2$. Quantum (a) and classical (d) are obtained at the same instant $t=20$. They exhibit some similarities (i.e., the shape of the regions of significant probability density, "ridges" in the topographical maps of (a) and (d)), but the difference - the presence of the interference patterns with $W(x, p)$ assuming negative values (marked with blue) is striking. Saturation of the size of the smallest patches is anticipated already at this early time, as the ridges of the classical probability density are narrower than in the corresponding quantum features. Saturation is even more visible in (c) taken at $t=60$ and $(\mathrm{d}), t=100$ (note change of scale). Sharpness of the classical features makes simulations going beyond $t=20$ unreliable, but quantum simulations can be effectivelly carried out much further, as the necessary resolution can be anticipated in advance from Eqs. (3.15) - (3.16).

Fig. 2. Difference between the classical and quantum average of the dispersion of momentum $\Delta^{2}=\left\langle p^{2}\right\rangle-\langle p\rangle^{2}$ is plotted in (a) for the same initial condition, but three different values of $\hbar$ in the model defined in Fig. 1, but with the parameter $a=0$. The instant when the departure between the classical and quantum averages becomes significant varies with $\hbar$ in a manner anticipated from Eqs. (3.5) and (3.6) for the Ehrenfest time, as is seen in the inset. Figure (b) shows the behaviors for the same value of $\hbar$, but for four different initial conditions. Inset appears to indicate that the typical variance difference $\delta$ varies only logarithmically with $\hbar$, although the large error bars (tied to the large systematic changes of behavior for different initial conditions) preclude one from arriving 
at a firmer conclusion. (See Karkuszewski, Zakrzewski, and Zurek, 2002, for further details and discussion).

Fig. 3. Schematic representation of the effect of decoherence on the Bloch sphere. When the interaction with the environment singles out pointer states located on the poles of the Bloch sphere, pure states (which lie on its surface) will evolve towards the vertical axis. This classical core is a set of all the mixtures of the pointer states.

Fig. 4. Information transfer in a c-not caricature of measurement, decoherence, and decoherence with noise. Bit-by-bit measurement is shown on the top. It is the fundamental logical circuit used to represent decoherence affecting the apparatus. Note that the direction of the information flow in decoherence - from the decohering apparatus and to the environment - differs from the information flow associated with noise. In short, as a result of decoherence environment is perturbed by the state of the system. Noise is - by contrast - perturbation inflicted by the environment. Preferred pointer states are selected so as to minimize the effect of the environment - to minimize the number of c-nots pointing from the environment at the expense of these pointing towards it.

Fig. 5. Time-dependent coefficients of the perturbative master equation for quantum Brownian motion. The parameters used in these plots (where the time is measure in units of $\Omega^{-1}$ ) are $\gamma / \Omega=0.05, \Gamma / \Omega=100, k_{B} T / \hbar \Omega=$ $10,1,0.1$. Plots on the right show the initial portion of the plots on the left - the initial transient - illustrating its independence of temperature (although higher temperatures produce higher final values of the coefficients). Plots on the right show that the final values of the coefficients strongly depend on temperature, and that the anomalous diffusion is of importance only for very low temperatures.

Fig. 6. Evolution of the Wigner function of the decohering harmonic oscillator. Note the difference between the rate at which the interference term disappears for the initial superposition of two minimal uncertainty Gaussians in position and in momenta.

Fig. 7. Predictability sieve in action. The plot shows purity $\operatorname{Tr} \rho^{2}$ for mixtures that have evolved from initial minimum uncertainty wavepackets with different squeeze parameters $s$ in an underdamped harmonic oscillator with $\gamma / \omega=10^{-4}$. Coherent states - which have the same spread in position as in momentum, $s=1-$ are clearly most predictable.

Fig. 8. Snapshots of a chaotic system with a double-well potential: $H=p^{2} / 2 m+A x^{4}-B x^{2}+C x \cos (f t)$. In the example discussed here $m=1, A=0.5, B=10$, $f=6.07$ and $C=10$ yielding the Lyapunov exponent $\Lambda \approx 0.45$ (see Habib, Shizume, and Zurek, 1998). All figures were obtained after approximately eight periods of the driving force. The evolution started from the same minimum uncertainty Gaussian, and proceeded according to the quantum Moyal bracket (a), the Poisson bracket (b), and (c) the Moyal bracket with decoherence (constant $D=0.025$ in Eq. (5.64)). In the quantum cases $\hbar=0.1$, which corresponds to the area of the rectangle in the image of the Wigner function above. Interference fringes are clearly visible in (a), and the Wigner function shown there is only vaguely reminiscent of the classical probability distribution in (b). Even modest decoherence $(D=0.25$ used to get (c) corresponds to coherence length $\left.\ell_{c}=0.3\right)$ dramatically improves the correspondence between the quantum and the classical. The remaining interference fringes appear on relatively large scales, which implies small scale quantum coherence.

Fig. 9. Classical and quantum expectation values of position $\langle x\rangle$ as a function of time for an example of Fig. 8. Evolution started from a minimum uncertainty Gaussian. Noticeable discrepancy between the quantum and classical averages appears on a timescale consistent with the Ehrenfest time $t_{\hbar}$. Decoherence - even in modest doses - dramatically decreases differences between the expectation values. 


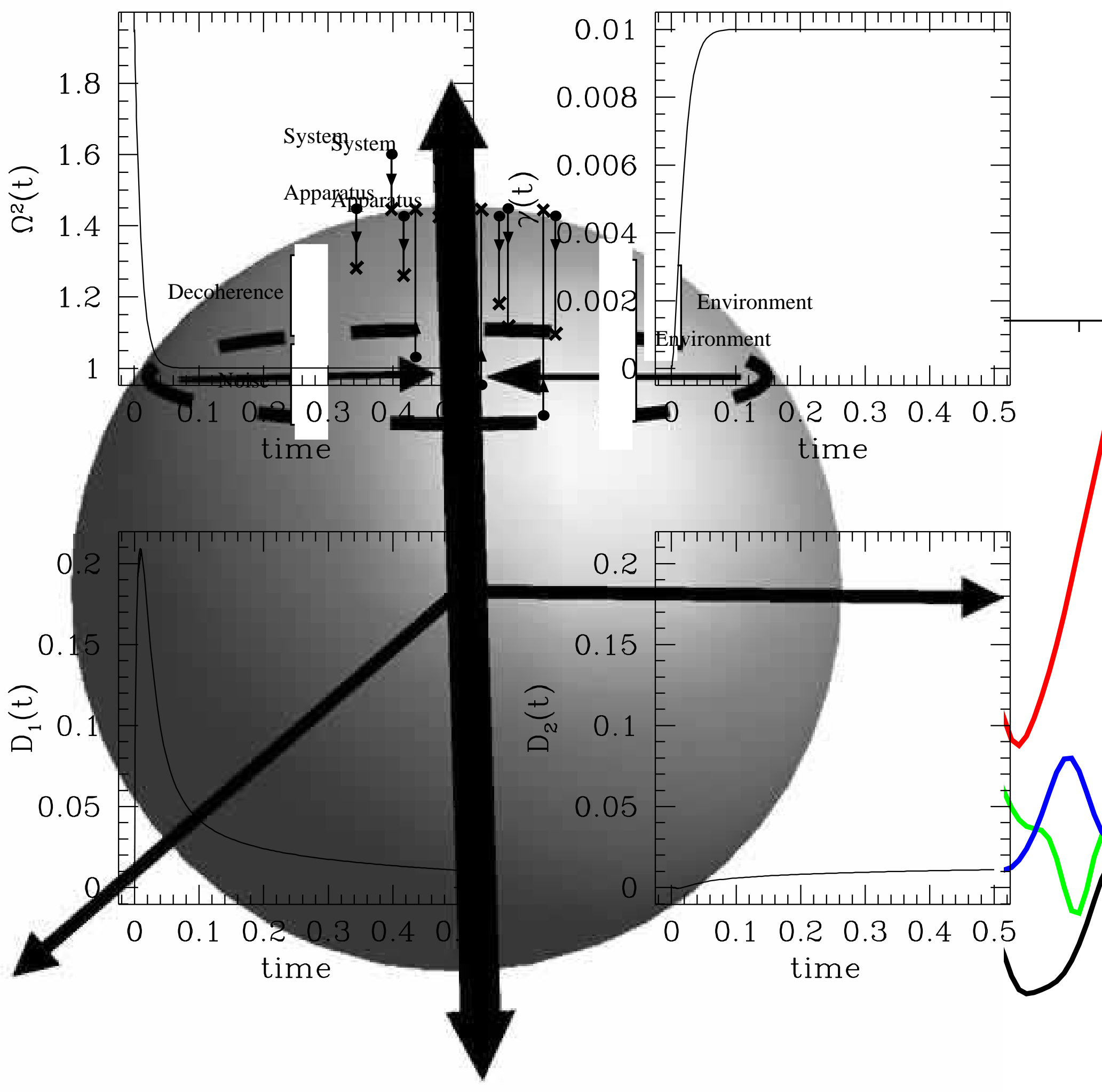




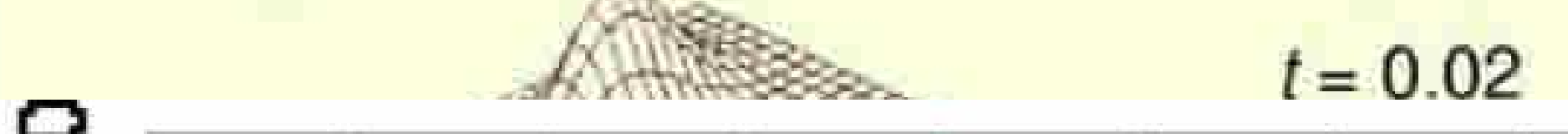

니
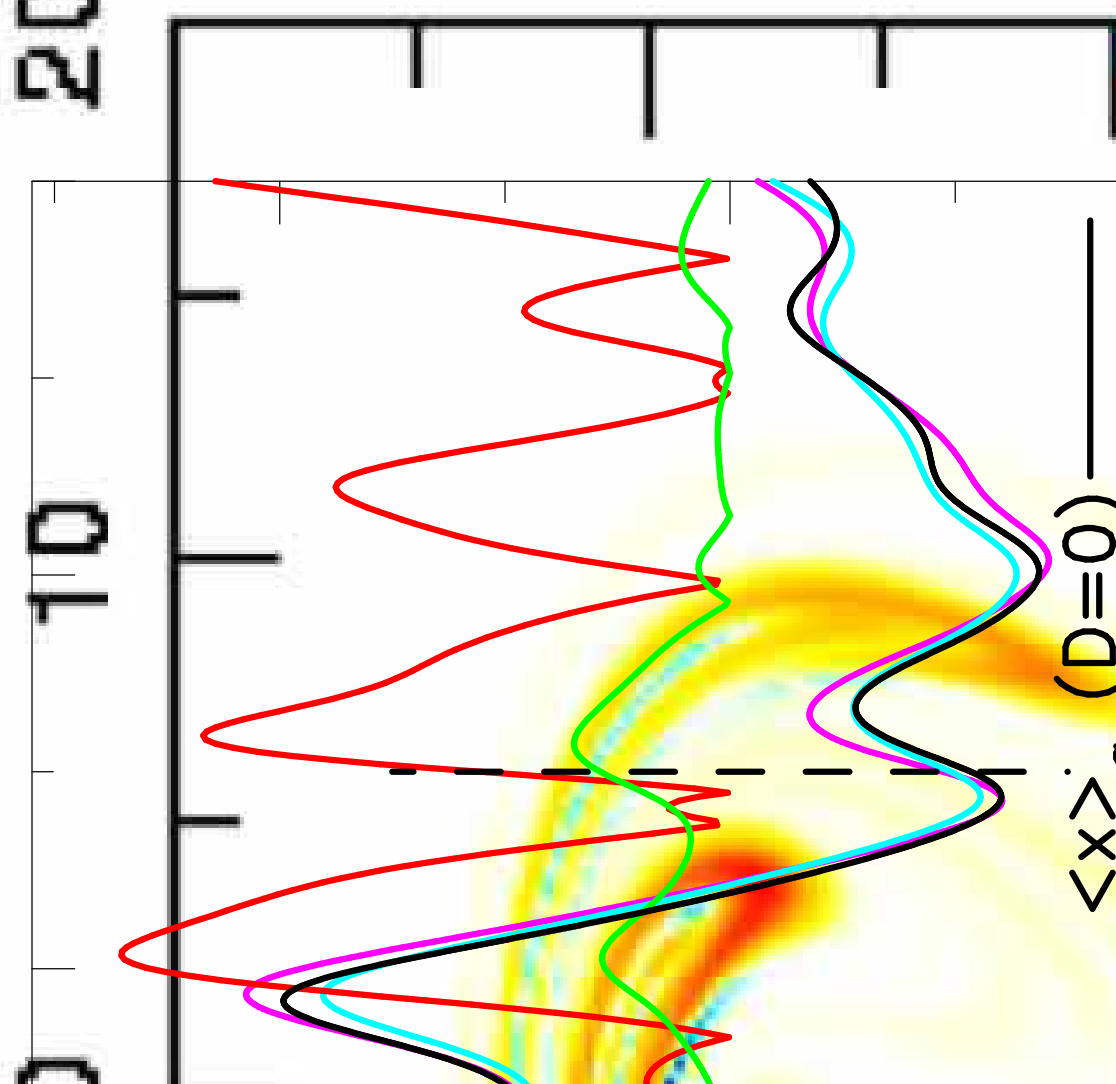

$a$

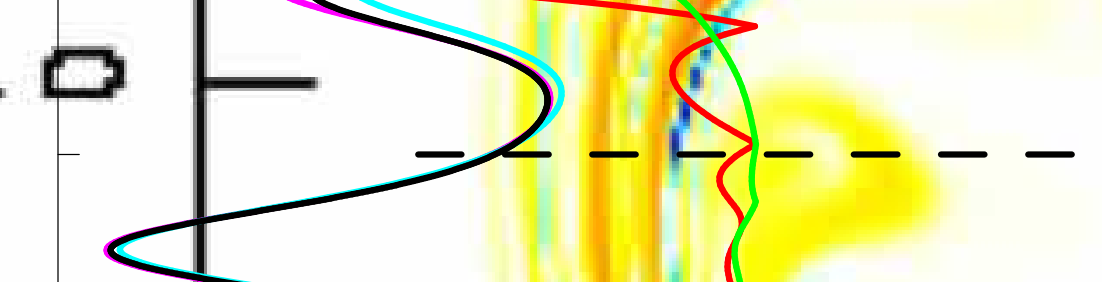

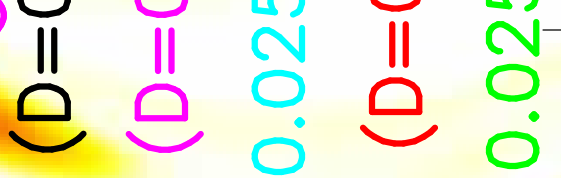

$\hat{x}_{\hat{v}}^{\sigma} \hat{x}^{0} \stackrel{\hat{v}^{0}}{\hat{x}^{\|}}$

$(0$

$\hat{\lambda}^{\sigma} \hat{v}$

$\mathrm{V}$
$\mathrm{V}$

$\frac{V}{*}{ }^{*}$

$\sim x$
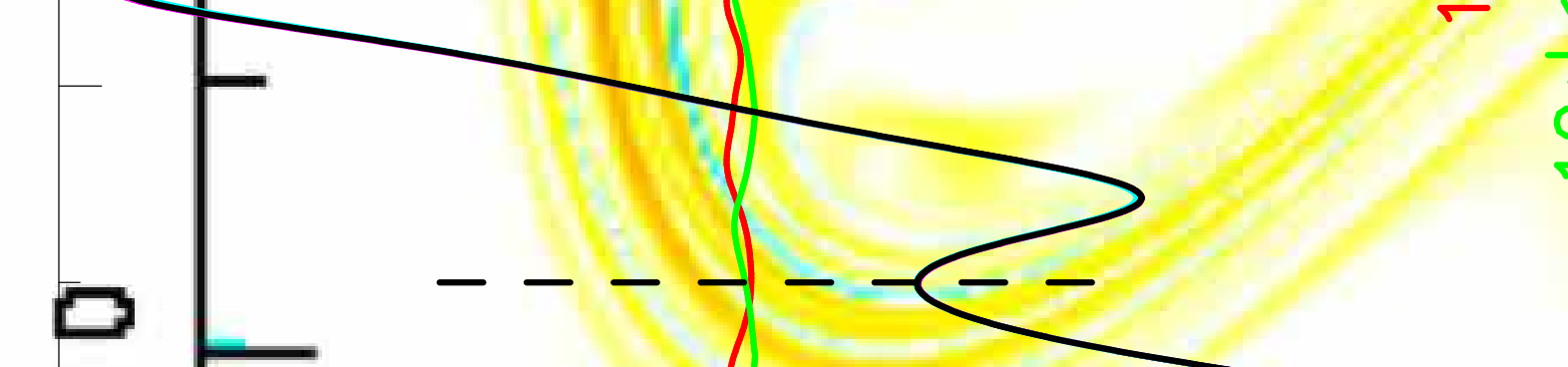

i

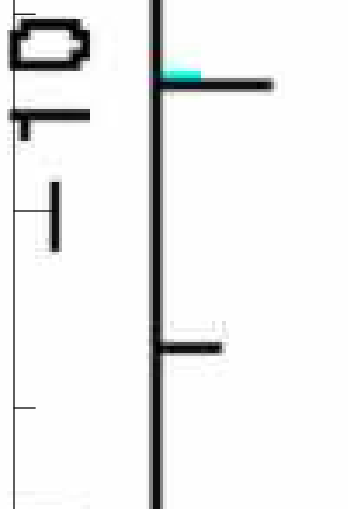

员 $1,1 / 1$,

I-E -4

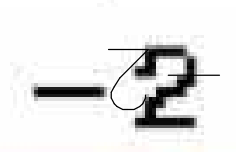

$\frac{9}{x}$

\section{$-02$ \\ 0 \\ 0.2 \\ 0.4 \\ 0.6}

